\title{
Elucidating the pathomechanism behind the neurocristopathy CHARGE syndrome
}

\section{Doctoral Thesis}

In partial fulfillment of the requirements for the degree

"Doctor rerum naturalium (Dr. rer. nat.)"

in the Molecular Medicine Study Program at the Georg-August University Göttingen

submitted by

Luisa Freese

born in

Halberstadt, Germany

Göttingen, 2017 


\section{Members of the Thesis Committee}

\section{Supervisor}

PD Dr. Silke Pauli

Institute of Human Genetics

University Medical Center Göttingen

\section{Second member of the Thesis Committee}

Prof. Steven A. Johnsen, PhD

Department of General, Visceral and Pediatric Surgery

University Medical Center Göttingen

Third member of the Thesis Committee

Prof. Dr. Ahmed Mansouri

Department of Molecular Cell Biology/Molecular Cell Differentiation

Max Planck Institute for Biophysical Chemistry, Göttingen

Date of Disputation: 


\section{AFFIDAVIT}

Herewith I declare that my doctoral thesis entitled: "Elucidating the pathomechanism behind the neurocristopathy CHARGE syndrome" has been written independently with no other sources and aids than quoted.

Göttingen, May 2017

Luisa Freese 


\section{List of Publications}

- Schulz Y, Freese L, Manz J, Zoll B, Volter C, Brockmann K, Bogershausen N, Becker J, Wollnik B, Pauli S (2014). CHARGE and Kabuki syndromes: a phenotypic and molecular link. Human molecular genetics 23(16): 4396-4405.

- A manuscript is in preparation that will contain parts of this doctoral thesis.

Parts of this doctoral thesis were presented at the following meetings:

- 25th Annual Meeting of the German Society of Human Genetics together with the Austrian Society of Human Genetics and the Swiss Society of Medical Genetics March 19-21, 2014, Essen, Germany

"CHARGE and Kabuki syndrome. Two related syndromes?" (poster presentation)

- 26th Annual Meeting of the German Society of Human Genetics together with the Austrian Society of Human Genetics and the Swiss Society of Medical Genetics April 15-17, 2015, Graz, Austria

"Chd7, the protein affected in CHARGE syndrome, regulates the neural crest cell guidance molecule Sema3a." (poster presentation) 


\section{Table of contents}

Table of contents ...................................................................................

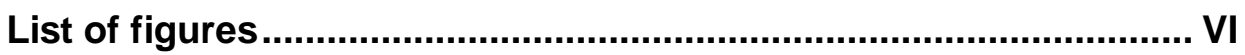

List of tables .................................................................................... VIII

Abbreviations.................................................................................. IX

Introduction .....................................................................................



1.1.2 Mutations in CHD7 lead to CHARGE syndrome ................................2

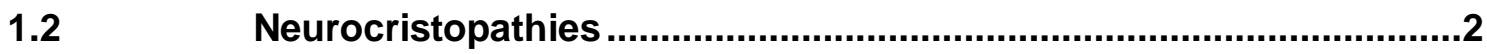

$1.3 \quad$ Neural crest cells - the fourth germ layer.........................................

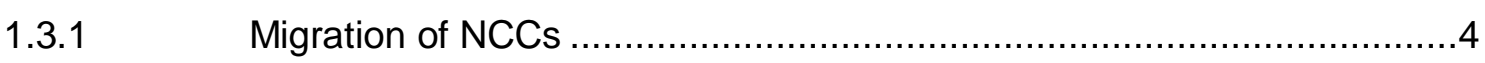

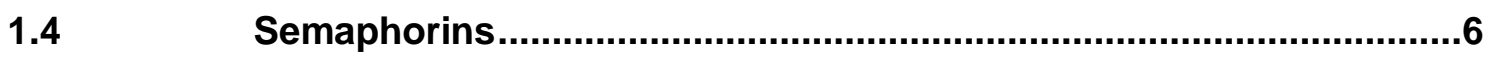

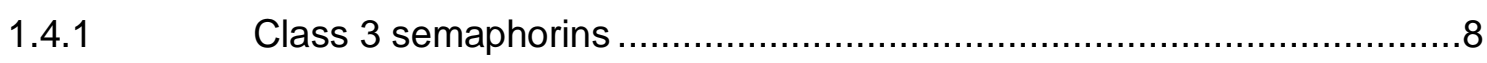

1.4.2 SEMA3A mutations contribute to the phenotype of

Kallmann syndrome ............................................................. 8

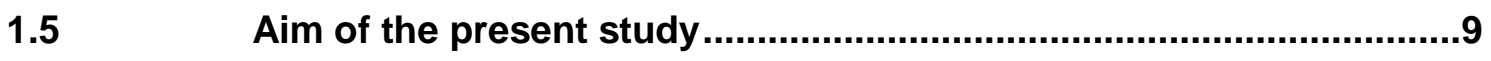

Materials....................................................................................10

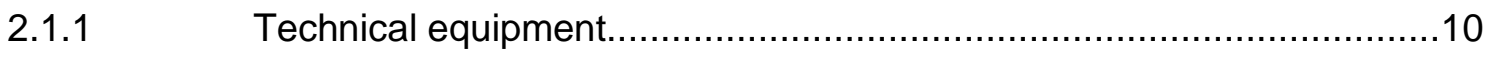

2.1.2 Consumable materials ............................................................. 12

2.1.3 Chemicals, biochemicals and reagents........................................ 13

2.1.4 Ready-to-use reaction systems ................................................ 16

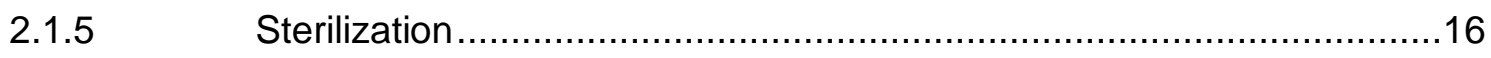

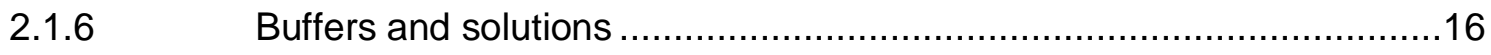

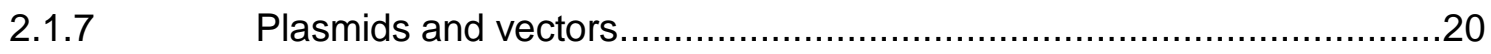

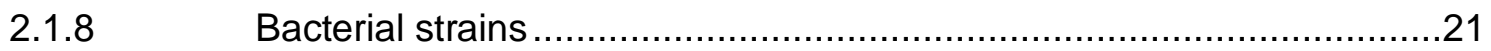

2.1.8.1 Media and agar plates for bacterial culture ...................................21 


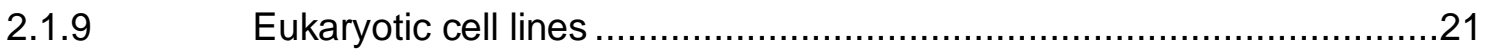

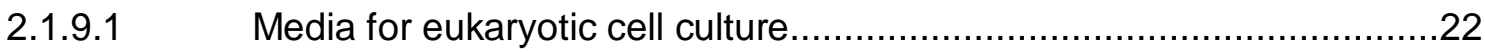

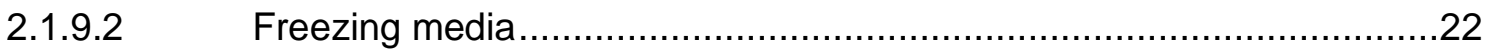

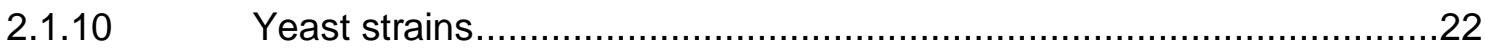

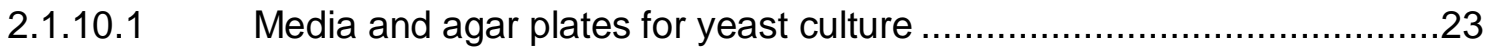

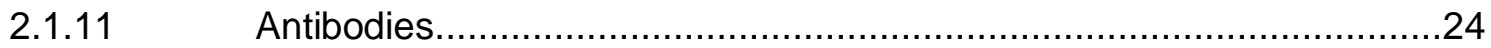

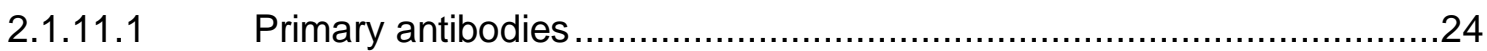





2.1.12.1 Synthetic oligonucleotides for genotyping PCR experiments ................25

2.1.12.2 Synthetic oligonucleotides for In-Fusion ${ }^{\circledR}$ experiments..........................25

2.1.12.3 Synthetic oligonucleotides for mutagenesis experiments .....................26

2.1.12.4 Synthetic oligonucleotides for mycoplasma contamination...................26

2.1.12.5 Synthetic oligonucleotides for patient screening ................................26

2.1.12.6 Synthetic oligonucleotides for RT-PCR experiments ............................27

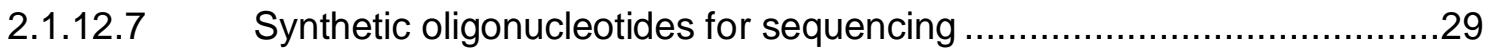

2.1.12.8 Synthetic oligonucleotides for qRT-PCR experiments........................29

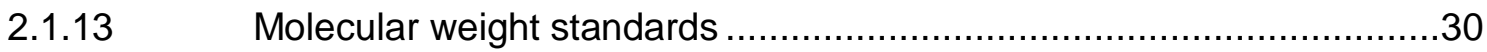

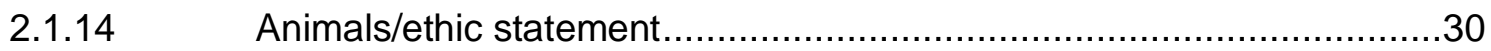

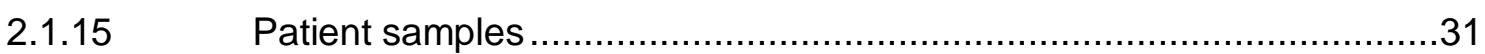

2.1.16 Databases / Online tools / Programs .............................................31

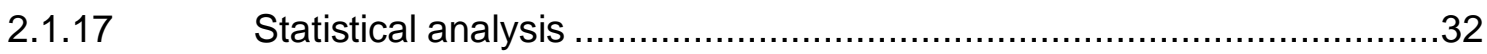

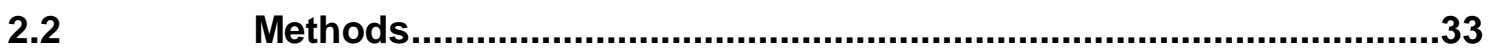

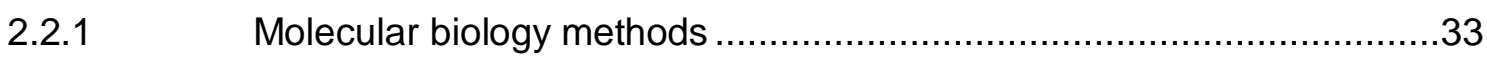

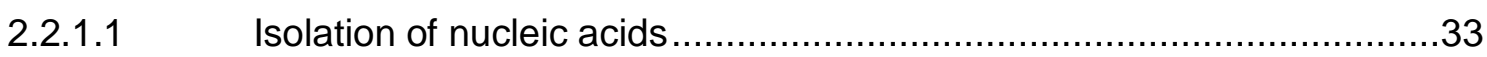

2.2.1.1.1 Isolation of genomic DNA for mouse genotyping ...............................33

2.2.1.1.2 Minipreparation of plasmid DNA ................................................

2.2.1.1.3 Endotoxin-free midipreparation of plasmid DNA ….............................34

2.2.1.1.4 Isolation of total RNA from cultured cells .........................................34

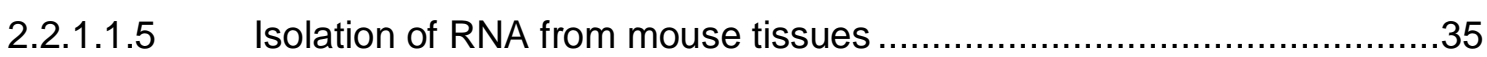

2.2.1.1.6 Determination of nucleic acid concentration..........................................

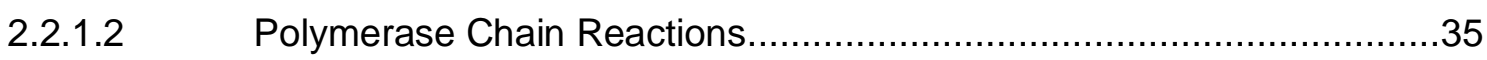

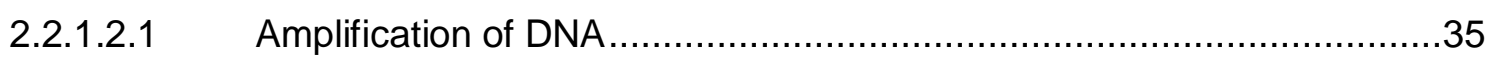

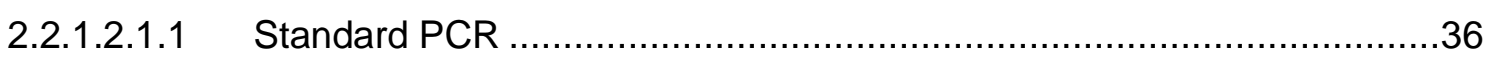




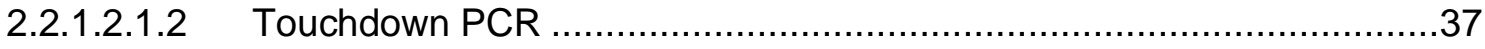

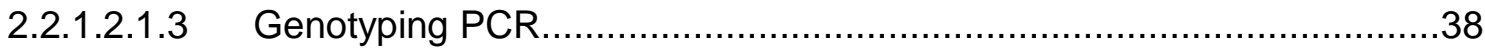

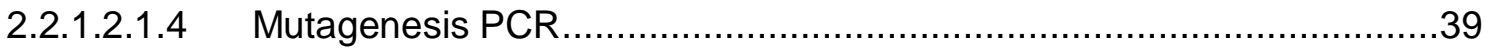

2.2.1.2.1.5 Mycoplasma contamination PCR ..................................................... 41

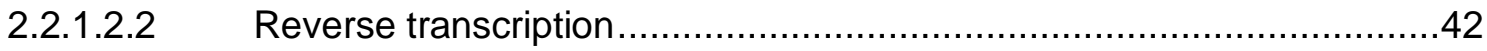

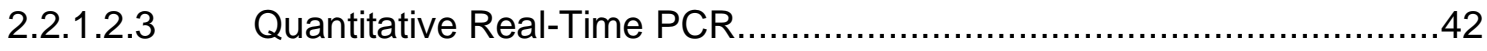

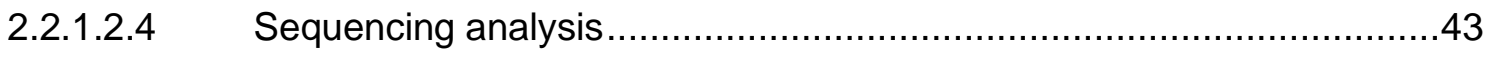

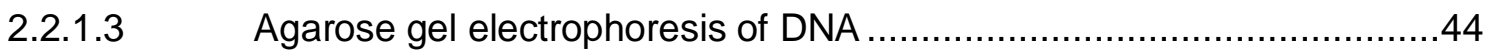

2.2.1.3.1 Purification of DNA fragments from agarose gels .............................45

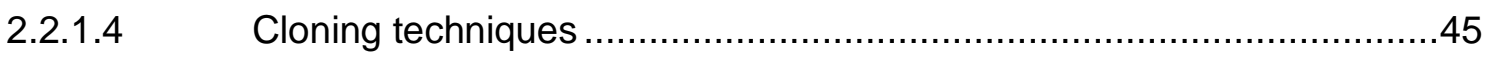

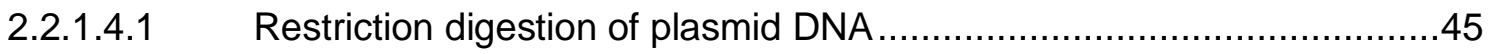

2.2.1.4.2 Cloning of DNA fragments using In-Fusion ${ }^{\circledR}$ HD Cloning Kit .................46

2.2.1.4.3 Transformation of competent cells ................................................46

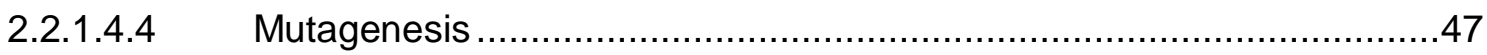

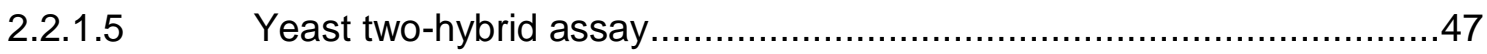

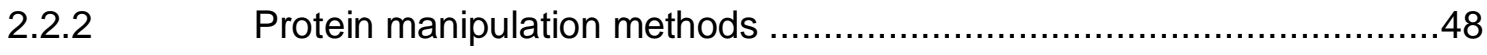

2.2.2.1 Protein isolation from eukaryotic cells ............................................. 48

2.2.2.2 Protein isolation from yeast cells.................................................. 48

2.2.2.3 Protein concentration from cell culture medium ................................49

2.2.2.4 Determination of protein concentration …........................................ 49

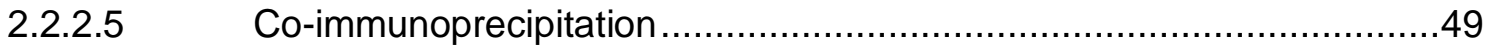

2.2.2.6 SDS polyacrylamide gel electrophoresis........................................50

2.2.2.7 Transfer of proteins from a polyacrylamide gel to a membrane.............51

2.2.2.8 Protein detection on membranes using antibodies...............................51

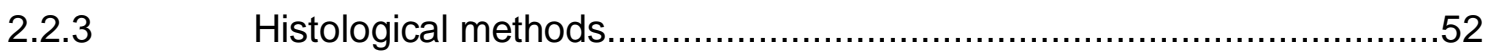

2.2.3.1 Dissection of mouse tissues and embryos ........................................ 52

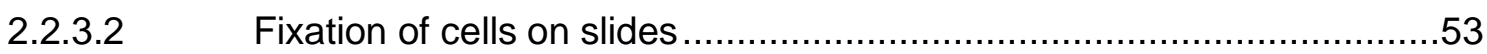

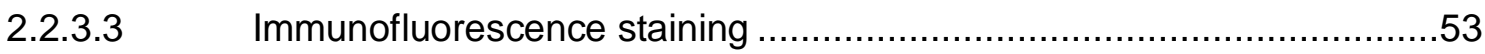

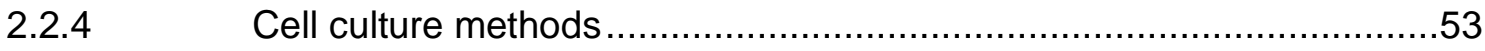

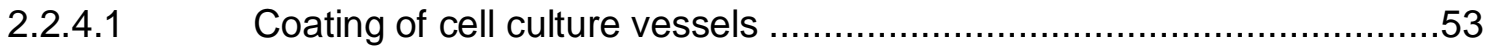

2.2.4.1.1 Preparation of fibronectin coated cell culture vessels..........................53

2.2.4.1.2 Preparation of matrigel coated cell culture vessels .............................54

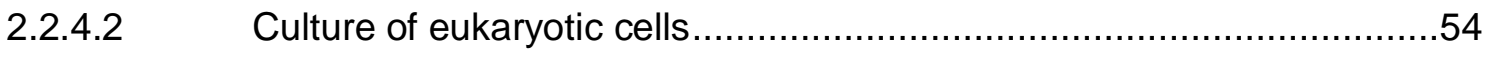

2.2.4.2.1 Culture and passaging of murine JoMa1 and JoMa1.3 cells ................54

2.2.4.2.2 Culture and passaging of murine 09-1 cells .....................................55 
2.2.4.2.3 Culture and passaging of human HEK293 cells ................................55

2.2.4.2.4 Cryopreservation and thawing of eukaryotic cells .............................56

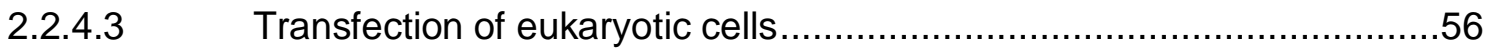

2.2.4.3.1 Transfection of plasmids into eukaryotic cells .................................56

2.2.4.3.2 Transfection of short interfering RNAs ............................................. 57

3.1.1 Molecular marker expression in murine JoMa cells.

3.1.2 Molecular marker expression in murine 09-1 cells

3.1.3 Chd7 expression in murine JoMa cells and O9-1 cells.

3.2 09-1 cells express semaphorin receptors and

class 3 semaphorins

3.3 Sema3a is expressed, processed and secreted by 09-1 cells and exists in monomeric and dimeric isoforms

3.4 Downregulation of Chd7 has influence on the expression level of Sema3a in 09-1 cells. .73

3.5 Chd7 co-immunoprecipitates with Sema3a in 09-1 cells .74

3.6 Detection of the non-synonymous SEMA3A variant c.2002A $>\mathrm{G}$ (p.I668V) in a CHD7-positive CHARGE syndrome patient.................76

3.7 Generation of SEMA3A plasmids .77

3.8 SEMA3A variants have no effect on the expression, processing and secretion as well as dimerization of SEMA3A. . .78

3.9 CHD7 co-immunoprecipitates with SEMA3A WT and

SEMA3A variants .83

3.10 CHD7 shows no direct interaction with SEMA3A WT or SEMA3A variants 
4.2 NCCs - which in vitro model to choose?

4.3 NCCs express class 3 semaphorins and secrete Sema3a

4.4 Chd7 regulates Sema3a expression and is associated with

Sema3a in NCCs.

Future perspectives. 


\section{List of figures}

Figure 1: Schematic representation of the CHD7 structure .........................................

Figure 2: Delamination and differentiation of NCCs ........................................... 4

Figure 3: Schematic representation of the protein structure of semaphorins and their main receptors.

Figure 4: Expression analysis of NCC, neuronal, glia, smooth muscle, melanocyte and chondrocyte marker genes in JoMa1 cells and JoMa1.3 cells

Figure 5: Expression analysis of NCC, neuronal, glia, smooth muscle, melanocyte and chondrocyte marker genes in 09-1 cells. 63

Figure 6: RT-PCR analysis of Chd7 expression in JoMa cells and 09-1 cells

Figure 7: Western blot analysis of Chd7 expression in JoMa cells and 09-1 cells ......64

Figure 8: Chd7 immunocytochemical staining of 09-1 cells. 66

Figure 9: Expression analysis of semaphorin receptors in 09-1 cells 67

Figure 10: Expression analysis of class 3 semaphorins in 09-1 cells .67

Figure 11: Sema3a immunocytochemical staining of 09-1 cells .68

Figure 12: Schematic representation of full-length Sema3a protein..... .69

Figure 13: Isoforms of Sema3a resulting from proteolytic processing and dimerization

Figure 14: Schematic representation of proteolytic processed and dimerized

Sema3a isoforms identified in concentrated medium as well as

cytoplasmic and nuclear protein extracts of 09-1 cells .

Figure 15: Analysis of the Chd7 expression in 09-1 cells after Chd7 downregulation...73

Figure 16: Analysis of the Sema3a expression in 09-1 cells after Chd7 downregulation 74

Figure 17: Co-immunoprecipitation of Chd7 with Sema3a in O9-1 cells .75

Figure 18: SEMA3A mutation found in a CHD7-positive CHARGE syndrome patient...77

Figure 19: Schematic representation of full length SEMA3A protein with identified mutation

Figure 20: Expression and proteolytic processing of transient overexpressed SEMA3A WT and SEMA3A variants in HEK293 cells ....

Figure 21: Secretion and dimerization of transient overexpressed SEMA3A WT and SEMA3A variants in HEK293 cells 
Figure 22: Co-immunoprecipitation of CHD7 with SEMA3A WT and SEMA3A variants in HEK293 cells 84

Figure 23: Expression of the full-length SEMA3A isoform in yeast cells .86

Figure 24: Schematic overview of the used CHD7 yeast two-hybrid assay plasmids....87

Figure 25: Yeast two-hybrid assay .88 


\section{List of tables}

Table 1: $\quad$ Sema3a isoforms detected in concentrated medium as well as cytoplasmic and nuclear protein extracts of 09-1 cells 


\section{Abbreviations}

\begin{tabular}{ll}
\hline $3^{\prime}$ & $\begin{array}{l}\text { 3' terminus of DNA } \\
5^{\prime}\end{array}$ \\
4 -OHT & 4-hydroxinus of DNA \\
$\%$ & percent \\
$\alpha$ & alpha \\
$\Delta$ & delta \\
+ & plus \\
- & minus \\
\pm & plus/minus \\
$>$ & greater than or replacement to \\
$<$ & less than \\
$\leq$ & less than or equal to \\
$\sim$ & tilde (approximately) \\
$\infty$ & infinity \\
$\circledast$ & registered trademark \\
TM & unregistered trademark
\end{tabular}

A

A

adenine or alanine

aa

amino acids

$\mathrm{Ab}$

antibody

Actg2

actin, gamma 2 , smooth muscle enteric

ADA2

adaptor 2

ADAM

a disintegrin and a metalloprotease domain

ADAMTS1

ADAM with thrombospondin motif $\underline{1}$

Ade

adenine

ATCC

American Type Culture Collection

ATP

adenosine triphosphate

ATPase

adenosine triphosphatase 
B

$\begin{array}{ll}\text { Baf47 } & \text { Brahma-associated factor } 47 \\ \text { bp } & \text { base pairs } \\ \text { BRG1 } & \text { Brahma-related gene 1 } \\ \text { BRK } & \text { brahma and kismet } \\ \text { BSA } & \text { bovine serum albumin }\end{array}$

C

${ }^{\circ} \mathrm{C}$

C

centigrade

c.

carboxyl-terminus or cytoplasmic protein extract or cytosine

$\mathrm{CaCl}_{2}$

cDNA reference sequence

cDNA

calcium chloride

CEE

complementary deoxyribonucleic acid

CHD

chicken embryo extract

CHD7 / Chd7

chromodomain helicase DNA-binding

ChIP

chromodomain helicase DNA-binding protein 7

Chromo

chromatin immunoprecipitation

CIL

chromodomain

$\mathrm{CM}$

contact inhibition of locomotion

CMV

concentrated cell culture medium

Cnn1

pCMV vector

$\mathrm{CO}_{2}$

calponin 1

carbon dioxide

Co-IP

co-immunoprecipitation

Col2a1

collagen, type II, alpha 1

CR1, 2, 3

conserved region 1, 2, 3

Cre

causes recombination

$\mathrm{Ct}$

threshold cycle

CUB

complement C1r/C1s-Uegf-Bmp1

Cy3

cyanine 3

D

d

day 


$\begin{array}{ll}\text { DAPI } & \text { 4',6-diamidino-2-phenylindole } \\ \text { dATP } & \text { deoxyadenosine triphosphate } \\ \text { dCTP } & \text { deoxycytidine triphosphate } \\ \text { ddH }{ }_{2} \mathrm{O} & \text { bi-distilled water } \\ \text { DEPC } & \text { diethyl pyrocarbonate } \\ \text { dGTP } & \text { deoxyguanosine triphosphate } \\ \text { DMEM } & \text { Dulbecco's Modified Eagle Medium } \\ \text { DMSO } & \text { dimethyl sulfoxide } \\ \text { DNA } & \text { deoxyribonucleic acid } \\ \text { DNase I } & \text { deoxyribonuclease I } \\ \text { dNTP } & \text { deoxynucleotide triphosphates } \\ \text { DO } & \text { dropout } \\ \text { DPBS } & \text { Dulbecco's Phosphate-Buffered Saline } \\ \text { DPBST } & \text { Dulbecco's Phosphate-Buffered Saline with Tween } 20 \\ \text { Dpnl } & \text { Diplococcus pneumonia I } \\ \text { DTT } & 1,4-\text { dithiothreitol } \\ \text { dTTP } & \text { desoxythymidin triphosphate }\end{array}$

E

$E$

EcoRl or embryonic day

EcoRI

Escherichia coli, strain R I

EDTA

ethylenediaminetetraacetic acid disodium salt dihydrate

EGF

epidermal growth factor

EMT

epithelial-to-mesenchymal transition

Epha3

Ephrin receptor A3

Epha5

Ephrin receptor A5

Epha7

Ephrin receptor A7

ESC

embryonic stem cell

ET

endothelin

et al.

et alii (and others)

\section{F}

FB

fibroblast 
FBS

FGF-2

FV/VIII fetal bovine serum

fibroblast growth factor-2

coagulation factor V/VIII homology-like

G

G

guanine

g

gram

$g$

gravitational acceleration $\left(9.81 \mathrm{~m} / \mathrm{s}^{2}\right)$

GAD

pGADT7 vector

GAP

guanosine triphosphatase (GTPase) activating protein

gDNA

genomic deoxyribonucleic acid

GDNF

glial cell line-derived neurotrophic factor

Gfap

glial fibrillary acidic protein

GFP

green fluorescent protein

GPI

glycosylphosphatidylinositol

H

h

$\mathrm{H}_{2} \mathrm{O}$

hour or human

$\mathrm{HA}$

water

$\mathrm{HCl}$

hemagglutinin

HEK

hydrochloric acid

HeLa

human embryonic kidney

His

Henrietta Lacks

Hprt

histidine

HRP

hypoxanthine-guanine phosphoribosyltransferase

horseradish peroxidase

I

I

isoleucine

ICC

immunocytochemistry

$\lg$

immunoglobulin

IPT

lg-like-plexin-transcription factors

ISH

in situ hybridization 
K

$\mathrm{kb}$

kilo base

$\mathrm{KCl}$

potassium chloride

$\mathrm{kDa}$

kilo Dalton

$\mathrm{KH}_{2} \mathrm{PO}_{4}$

potassium dihydrogen phosphate

$\mathbf{L}$

।

liter

LB

lysogeny broth

LDS

lithium dodecyl sulfate

Leu

leucine

LiAc

lithium acetate

LIF

leukemia inhibitory factor

$-\mathrm{LT}$

without leucine, tryptophan

- LTHA

without leucine, tryptophan, histidine, adenine

\section{M}

$\mu$

$\mu \mathrm{g}$

$\mu l$

$\mu \mathrm{M}$

$\mu \mathrm{m}$

M

$\mathrm{m}$

MAM

MEM

MES

micro $\left(10^{-6}\right)$

g

microgram

microliter

micromolar

micrometer

molar

milli $\left(10^{-3}\right)$ or mouse

meprin-like

minimum essential medium

2-(N-morpholino)ethanesulfonic acid

$\mathrm{mg}$

milligram

$\mathrm{MgCl}_{2}$

magnesium chloride

MIM

Mendelian Inheritance in Man

$\min$

minute

$\mathrm{ml}$

milliliter

$\mathrm{mM}$

millimolar 


$\begin{array}{ll}\text { mol } & \text { mole } \\ \text { MWCO } & \text { molecular weight cut-off } \\ \text { Myco } & \text { mycoplasma }\end{array}$

\section{$\mathbf{N}$}

$\mathrm{N}$

nuclear protein extract or amino-terminus

$\mathrm{n}$

nano $\left(10^{-9}\right)$

$\mathrm{NaCl}$

sodium chloride

$\mathrm{Na}_{2} \mathrm{HPO}_{4}$

disodium hydrogen phosphate

$\mathrm{NaOH}$

sodium hydroxide

NC

negative control

NCC

neural crest cell

$\mathrm{N}-\mathrm{CoR}$

nuclear receptor co-repressor

NEAA

non-essential amino acids

Nefh

neurofilament, heavy polypeptide

ng

nanogramm

$\mathrm{Ngfr}$

nerve growth factor receptor (previously named P75)

NM

NCBI accession number

$\mathrm{nM}$

nanomolar

NP

NCBI reference sequence for proteins

Nrp1

neuropilin 1

Nrp2

neuropilin 2

n.s.

not significant

$\mathbf{0}$

OD

optical density

P

p

protein

p.

protein reference sequence

P75

nerve growth factor receptor

$\mathrm{Pa}$

Pascal, unit of pressure

Pax3

paired box 3 


$\begin{array}{ll}\text { PBAF } & \text { polybromo- and BRG1-associated factor containing complex } \\ \text { PBS } & \text { phosphate buffered saline } \\ \text { PC } & \text { positive control } \\ \text { pCMV } & \text { plasmid cytomegalovirus } \\ \text { PCR } & \text { polymerase chain reaction } \\ \text { PDGF } & \text { platelet derived growth factor } \\ \text { Pdgfc } & \text { platelet-derived growth factor C } \\ \text { PEG } & \text { polyethylene glycol } \\ \text { PFA } & \text { paraformaldehyde } \\ \text { pH } & \text { potential of hydrogen } \\ \text { PLA } & \text { proximity ligation assay } \\ \text { PIxna1 } & \text { Plexin A1 } \\ \text { PNS } & \text { peripheral nervous system } \\ \text { PSI } & \text { plexin-semaphorin-integrin } \\ \text { PVDF } & \text { polyvinylidene fluoride }\end{array}$

Q

q long arm of the chromosome

qRT quantitative real-time PCR

qRT-PCR quantitative real-time PCR

$\mathbf{R}$

$\mathrm{R}$

arginine

RbBP5

retinoblastoma-binding protein 5

RNA

ribonucleic acid

RT room temperature

RT-PCR real-time PCR

S

S

Sfil

$S$ second

SANT switching-defective protein 3 (SWI3), adaptor 2 (ADA2), nuclear receptor co-repressor (N-CoR), transcription factor (TF) IIIB 
SDF1

SDS

SDS-PAGE

SEMA / Sema

SEMA3A / Sema3a

SEMA3C / Sema3c

SEMA3D / Sema3d

SEMA3E / Sema3e

seq

Sfil

SiRNA

Snai1

Snai2

SNF2

S.O.C.

Sox9

Sox10

SRY

SWI2

SWI3 stromal cell-derived factor 1

sodium dodecyl sulfate

sodium dodecyl sulfate polyacrylamide gel electrophoresis

Semaphorin

sema domain, immunoglobulin domain (lg), short basic domain, secreted, (semaphorin) 3a

sema domain, immunoglobulin domain (lg), short basic domain, secreted, (semaphorin) 3c

sema domain, immunoglobulin domain (lg), short basic domain, secreted, (semaphorin) 3d

sema domain, immunoglobulin domain (lg), short basic domain, secreted, (semaphorin) 3e

sequencing

Streptomyces fimbriatus I

short interfering RNA

snail family zinc finger 1

snail family zinc finger 2

sucrose non-fermentable 2

super optimal broth with catabolite repression

sex determining region $Y$ (SRY)-box 9

sex determining region $Y(S R Y)$-box 10

sex determining region of chromosome $Y$

switch 2

switching-defective protein 3

$\mathbf{T}$

$\mathrm{T}$

Taq

TBE

TBP

TBS

TBST

TE

TF IIIB threonine or thymine

Thermus aquaticus

tris-borate-EDTA

TATA box binding protein

Tris-buffered saline

tris-buffered saline with tween 20

Tris-EDTA

transcription factor IIIB 
TK

Tris

Trp

Twist1

Tyr

U

U

UV

V

$\mathrm{V}$

VEGF

Vegfc tyrosine kinase

tris(hydroxymethyl)aminomethane

tryptophan

twist basic helix-loop-helix transcription factor 1

tyrosinase

Unit (enzyme activity)

ultraviolet light

valine or Volt

vascular endothelial growth factor

vascular endothelial growth factor $\mathrm{C}$

W

W tryptophan

WB western blot

Whi Whirligig

Wnt1 Wnt family member 1

WT wild type

$\mathrm{w} / \mathrm{v} \quad$ weight/volume

$\mathbf{X}$

x $g$ multiple of gravitational acceleration

Y

$\mathrm{Y} 2 \mathrm{H}$

yeast two-hybrid

YNB

yeast nitrogen base 


\section{$1 \quad$ Introduction}

\section{$1.1 \quad$ CHARGE Syndrome}

\subsubsection{Chromodomain helicase DNA-binding protein 7}

Chromodomain helicase DNA-binding (CHD) proteins play a role in transcription activation and repression by ATP (adenosine triphosphate)-dependent chromatin remodeling (Kim et al., 2008). For this function, all members of the CHD protein family share at the $\mathrm{N}$-terminus two tandem chromodomains (chromatin organization modifier domains), followed by a SWI2/SNF2 (switch 2/sucrose non-fermentable 2)-like ATPase/helicase domain (Woodage et al., 1997; Flaus et al., 2006; Flanagan et al., 2007). In humans, the CHD protein family consists of nine members who are divided into three subgroups according to their amino acid sequence and additional functional protein domains. CHD7 is one of the nine CHD family members and belongs to subgroup III. Characteristics of this subgroup are three conserved regions (CR1-CR3), a SANT (switching-defective protein 3 (SWI3), adaptor 2 (ADA2), nuclear receptor co-repressor (N-CoR), transcription factor (TF) IIIB) domain and two tandem BRK (brahma and kismet) domains (Hall and Georgel, 2007; Marfella and Imbalzano, 2007) (Figure 1). The human CHD7 gene (MIM 608892) is located on chromosome 8 (8q12.2) and encodes 38 exons. It is highly conserved across species and orthologs have been identified in mouse, chicken, zebrafish, Xenopus laevis and others (Bosman et al., 2005; Aramaki et al., 2007; Bajpai et al., 2010).

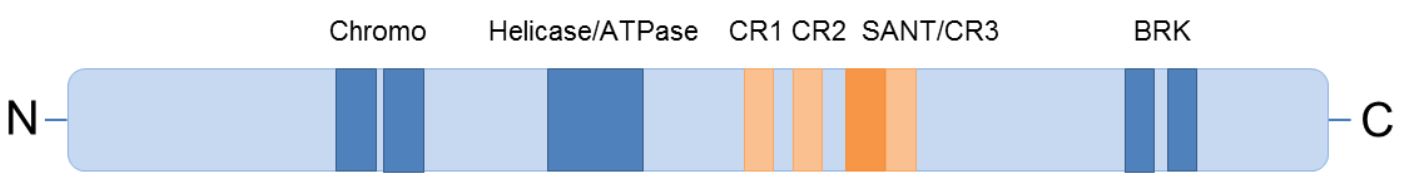

Figure 1: Schematic representation of the CHD7 structure. CHD7 is one of nine members of the CHD family that plays a role in controlling gene expression by ATP-dependent chromatin remodeling. CHD7 consists of two N-terminal chromodomains, a SWI2/SNF2-like ATPase/helicase domain, three conserved regions namely CR1, CR2 and CR3, a SANT domain and two BRK domains. Modified from Schulz et al. (2014a). 


\subsubsection{Mutations in CHD7 lead to CHARGE syndrome}

CHARGE syndrome (MIM 214800) is an autosomal dominant congenital malformation disorder known for its clinical variability. Reports of patients who were later found to have CHARGE were firstly published in 1979 independent by Hall (1979) and Hittner et al. (1979). Two years later, in 1981, Pagon et al. suggested for this disorder the acronym CHARGE. The acronym describes the main features seen in patients namely coloboma, heart defects, atresia of the choanae, retarded growth and development, genital hypoplasia and ear anomalies/deafness (Pagon et al., 1981). The phrase 'CHARGE association' was renamed 2004 into 'CHARGE syndrome' after identification of CHD7 mutations as the major disease cause (Vissers et al., 2004; Aramaki et al., 2006; Jongmans et al., 2006; Lalani et al., 2006; Sanlaville et al., 2006; Wincent et al., 2008). Blake et al. (1998) defined clinical criteria for the diagnosis of CHARGE syndrome. They were updated by Verloes (2005) defining coloboma, atresia of the choanae and hypoplastic semicircular canals as major signs and rhombencephalic dysfunction, hypothalamo-hypophyseal dysfunction, abnormal middle or external ear, malformations of mediastinal organs and mental retardation as minor signs. The incidence varies from 1:8500 to 1:10,000 (Blake et al., 1998; Issekutz et al., 2005). In the majority of patients with the suspected diagnosis CHARGE syndrome heterozygous mutations in the CHD7 gene are found. Most of them are nonsense or frameshift mutations (due to small deletions or insertions), but also missense mutations can occur (Janssen et al., 2012). To date, over 500 different pathogenic mutations have been identified which are distributed throughout the coding sequence as well as in some intronic sequences (Aramaki et al., 2006; Jongmans et al., 2006; Lalani et al., 2006; Sanlaville et al., 2006; Sanlaville and Verloes, 2007; Vuorela et al., 2007; Janssen et al., 2012; Martin, 2015). Most of them are de novo mutations, but also somatic and germline mosaicism in a parent were reported in families with more than one affected child (Jongmans et al., 2006; Delahaye et al., 2007; Jongmans et al., 2008; Pauli et al., 2009).

\section{$1.2 \quad$ Neurocristopathies}

Alterations in the complex succession of events required for induction, proliferation, migration and final differentiation of neural crest cells resulting in a large group of birth defects, which were firstly termed by Bolande (1974) neurocristopathies. The classification 
of syndromes as a neurocristophathy evolved over the years now including numerous congenital malformation syndromes such as CHARGE, Kallmann, DiGeorge, TreacherCollins, Waardenburg or Hirschsprung's disease (Siebert et al., 1985; Etchevers et al., 2006, 2007; Jones et al., 2008; Passos-Bueno et al., 2009; Keyte and Hutson, 2012). Interestingly, already in 1985, Siebert et al. postulated the belonging of CHARGE syndrome to the neurocristopathies (Siebert et al., 1985) because of the affected structures. The most common clinical features seen in neurocristopathies include craniofacial defects, hearing loss, pigmentation and cardiac defects as well as missing of enteric ganglia (Mayor and Theveneau, 2013).

\section{$1.3 \quad$ Neural crest cells - the fourth germ layer}

Neural crest cells (NCCs) are a population of multipotent and migratory progenitors found in all vertebrate embryos (Knecht and Bronner-Fraser, 2002; Milet and Monsoro-Burq, 2012). During embryogenesis they arise from the neural plate border which is induced by signaling between the neural and the non-neural ectoderm and from the underlying paraxial mesoderm (Gammill and Bronner-Fraser, 2003). The neural plate borders elevate during neurulation and cause the neural plate to folds over itself to form the neural tube (Gammill and Bronner-Fraser, 2003). In the neural plate border as well as in neighboring structures neural plate border specifier genes are upregulated (Bronner and LeDouarin, 2012). A further refinement of the border region results from the activation of neural crest specifiers, genes allowing NCCs the delamination from the neural folds or neural tube, the migration and differentiation into various derivatives (Bronner and LeDouarin, 2012) (Figure 2). NCCs migrate from the closing neural folds or from the dorsal neural tube through the embryo, following specific pathways to contribute to the formation of different organs and tissues like skin, heart or peripheral nervous system (PNS) (Gammill and Bronner-Fraser, 2003; Kuriyama and Mayor, 2008). Because of their ability to differentiate into various cell types like melanocytes, craniofacial cartilage and bone, smooth muscle, peripheral and enteric neurons and glia (Kuriyama and Mayor, 2008; Dupin and Sommer, 2012; Prasad et al., 2012) (Figure 2), they were termed the 'fourth germ layer' (Hall, 2000). NCCs are divided into the cranial (cephalic), cardiac, trunk and enteric NCCs. The cranial NCCs contribute to craniofacial structures like bones and cartilages of the face and neck as well as tendons, muscles and connective tissue of the ear, eye, teeth and blood vessels (Mayor and Theveneau, 2013). In addition, they form most of the cranial PNS and 
modulate brain growth and patterning (Mayor and Theveneau, 2013). The cardiac NCCs are a subpopulation of the cranial NCCs and essential for the septation of the heart outflow tract (Mayor and Theveneau, 2013). The trunk NCCs form pigment cells, the dorsal root and sympathetic ganglia of the PNS and endocrine cells of the adrenal gland, whereas the enteric NCCs form the enteric PNS of the gut, which controls the digestive track (Mayor and Theveneau, 2013).

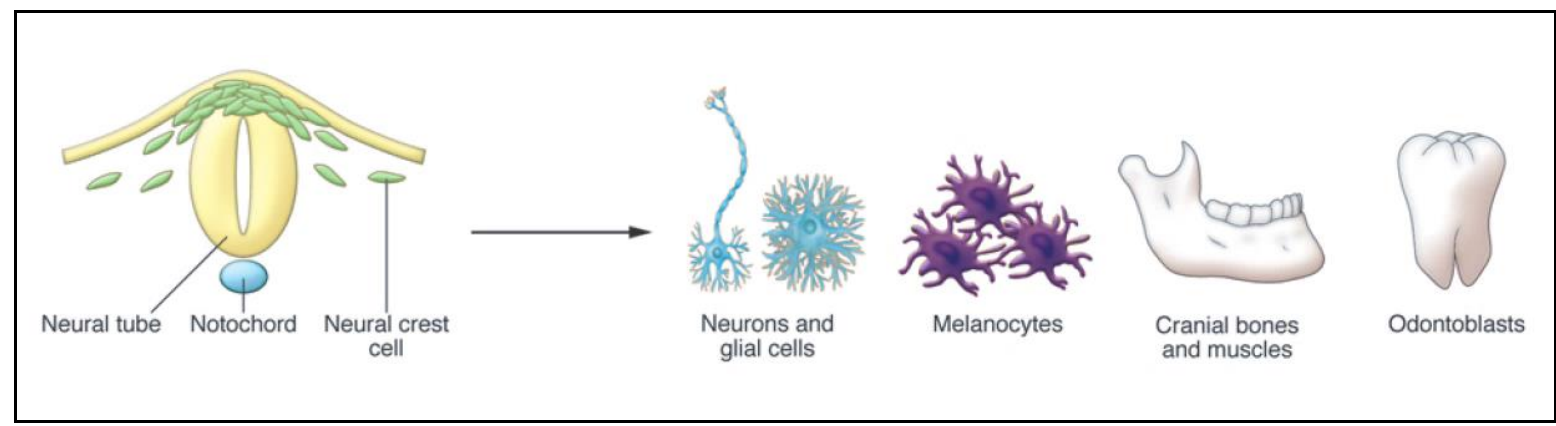

Figure 2: Delamination and differentiation of NCCs. NCCs delaminate from the closing neural folds or from the dorsal neural tube and differentiate into neurons of the peripheral nervous system, glial cells, melanocytes, cranial bones and muscles, odontoblasts or other cell types. Modified from Acloque et al. (2009).

\subsubsection{Migration of NCCs}

The migration of NCCs is a complex and multi-step process. Interestingly, Bajpai et al. (2010) demonstrated in humans and in the animal model Xenopus laevis an essential role for CHD7 in the formation and migration of NCCs by activating neural crest specifier genes as the transcription factors SNAI2 (snail family zinc finger 2, previously named Slug), TWIST (twist basic helix-loop-helix transcription factor) and SOX9 (SRY(sex determining region Y)-box containing gene 9). NCCs originate at the border between the neural and the non-neural ectoderm along the anteroposterior length of the developing embryo (Gong, 2014). Before NCCs are able to delaminate from the closing neural folds or from the dorsal neural tube, they undergo a process called epithelial-to-mesenchymal transition (EMT) in which the NCCs lose their cell-cell contacts, reorganize their cytoskeleton and acquire a motile phenotype (Kirby and Hutson, 2014). Premigratory NCCs form an epithelium with a typical apical-basal polarity which is surrounded by an underlying basement membrane (Kerosuo and Bronner-Fraser, 2012). During EMT, the polarity gets lost due to a progressive replacement of tight junctions by gap junctions 
(Kuriyama and Mayor, 2008). Furthermore, a shift from type I to type II cadherins, a group of transmembrane proteins, results in a lower adhesiveness of the cells which allows them to increase their motility (Mayanil, 2013). Kerosuo and Bronner-Fraser (2012) point out that extracellular matrix proteins, such as ADAM proteins (proteins containing a disintegrin and a metalloprotease domain) and matrix metalloproteinases induce a breakdown of the basement membrane to allow migration. After undergoing EMT, the NCCs initially delaminate as a continuous wave which quickly splits into discrete streams ventrally down the embryo displaying a range of migratory behaviors (Shellard and Mayor, 2016). Some NCCs exhibit an individual migratory behavior. But although the NCCs undergo EMT, most of them migrate together, either as chains, groups or even single sheets (Kulesa et al., 2010; Theveneau and Mayor, 2012; Shellard and Mayor, 2016). A process called contact inhibition of locomotion (CIL) prevent the NCCs during migration from overlapping with each other (Mayor and Carmona-Fontaine, 2010). After contact with one another, the migrating NCCs momentarily stop and favors dispersion in the opposite direction (Mayor and Carmona-Fontaine, 2010). Nevertheless, the chemoattractant mediated phenomenon of co-attraction allows collective migration of the NCCs in spite of reduced cell-cell adhesion due to EMT and dispersion of the cells induced by CIL (Carmona-Fontaine et al., 2011). The split of NCCs into distinct streams and their precise targeting to their destinations are controlled by a plethora of positive and negative regulators including signaling molecules and mediated via binding of external ligands to receptors on the NCC surface (Theveneau and Mayor, 2012). Negative guidance cues are located the borders of the migration pathways whereas the locations of positive guidance cues are along the pathways or within the NCC target regions (Theveneau and Mayor, 2012). Some of the main signals regulating NCC migration are semaphorins, ephrins/Ephs, slits, ETs (endothelins), SDF1 (stromal cell-derived factor 1), VEGFs and PDGFs (vascular endothelial and platelet-derived growth factors), FGFs (fibroblast growth factors) and GDNF (glial cell line-derived neurotrophic factor) (Theveneau and Mayor, 2012). Schulz et al. (2014b) performed a genome-wide microarray expression analysis on whole wildtype and Chd7 deficient (Chd7 $7^{\text {Whi/t }}$ and $\mathrm{Chd} 7^{\text {Whi/Whi}}$ ) mouse embryos of the Whirligig mouse line at E9.5, a point in time of NCC migration. They identified a misregulation of genes involved in the migration and guidance of NCCs such as the semaphorins Sema3a, $3 c$ and $3 d$, the ephrin receptors Epha3, Epha5 and Epha7 and the growth factors Vegfc and Pdgfc (vascular endothelial and platelet-derived growth factor $\mathrm{C}$ ) that further underlines the important role of CHD7 in NCC development (Schulz et al., 2014b). 


\section{$1.4 \quad$ Semaphorins}

Semaphorins are a large family of phylogenetically conserved guidance cues and were originally identified as molecules that control axon pathfinding during the development of the nervous system (Kolodkin et al., 1993). They are involved in many biological processes, including the regulation of immune responses (Suzuki et al., 2008; Takamatsu and Kumanogoh, 2012), cardiogenesis (Toyofuku et al., 2007; Toyofuku et al., 2008), angiogenesis (Serini et al., 2003) and vasculogenesis (Gu et al., 2005) as well as oncogenesis (Sekido et al., 1996). Furthermore, Semaphorins act as guidance cues for a range of migrating cells like the NCCs (Eickholt et al., 1999). In these processes semaphorins can function either as positive or as negative guidance cues (Tamagnone and Comoglio, 2004). To date, more than 20 semaphorin types, secreted or membraneassociated proteins, have been discovered. All semaphorins are characterized by the presence of a conserved extracellular amino-terminal Sema (Semaphorin) domain, important for dimerization and binding specificity with receptors, followed by a PSI (plexinsemaphorin-integrin) domain and a C-terminus domain, which confers class specific features, such as basic, Ig (immunoglobulin)-like and thrombospondin domains (Janssen et al., 2010; Liu et al., 2010; Nogi et al., 2010). Semaphorins are grouped into eight subfamily classes on the basis of structural and amino acid sequence similarity, containing invertebrate (classes 1 and 2), vertebrate (classes 3-7) and viral semaphorins (class V) (Jongbloets and Pasterkamp, 2014) (Figure 3). Semaphorins of the classes 2, 3 and $\mathrm{V}$ are secreted proteins while the other semaphorins are transmembrane proteins (classes 1, 4, 5 and 6) or GPI (glycosylphosphatidylinositol)-linked proteins (class 7) (Jongbloets and Pasterkamp, 2014). Nevertheless, membrane-associated semaphorins of the classes 4 and 7 can be further processed into soluble proteins by proteolytic degradation (Neufeld et al., 2012). Although additional receptors as TK (tyrosine kinase) receptors or integrins have been described, the main semaphorin receptors are plexins and neuropilins (Winberg et al., 1998; Takahashi et al., 1999; Tamagnone et al., 1999) (Figure 3). The nine vertebrate plexins are grouped into four subfamilies (A-D) and serve for the most semaphorins as direct binding receptors (Jongbloets and Pasterkamp, 2014). However, some secreted semaphorins, like most of class 3 semaphorins, require the presence of one of the two in vertebrates identified neuropilins (Nrp1 or Nrp2) as ligandbinding co-receptor (Jongbloets and Pasterkamp, 2014). 




Figure 3: Schematic representation of the protein structure of semaphorins and their main receptors. Left column: Semaphorins are grouped into eight subfamily classes on the basis of structural and amino acid sequence similarity. The classes 1 and 2 contain invertebrate semaphorins, the classes 3-7 vertebrate semaphorins and the class $V$ viral semaphorins. Semaphorins of the classes 2 and 3 are secreted proteins, the semaphorin classes 1, 4, 5 and 6 contain transmembrane proteins and the class 7 semaphorins are GPI-linked proteins. The membrane-associated semaphorins of the classes 4 and 7 can be processed into soluble proteins by proteolytic degradation indicated with scissors. All semaphorins are characterized by a Sema domain, a PSI domain and a specific C-terminus domain. Middle column: The main receptors for semaphorins are the plexins and neuropilins. The four subfamilies (A-D) of the nine vertebrate plexins, the two vertebrate neuropilins (Nrp 1 and Nrp 2) and their structures are shown. Right column: The structural conserved domains are drawn in different shapes and colors as indicated in the figure. Domains abbreviations: sema, semaphorin; PSI, plexin-semaphorin-integrin; Ig-like, immunoglobulin-like; GPI, glycosylphosphatidylinositol; IPT, Ig-like-plexin-transcription factors; GAP, guanosine triphosphatase (GTPase) activating protein; CUB, complement C1r/C1sUegf-Bmp1; FV/VIII, coagulation factor V/VIII homology-like; MAM, meprin-like. Modified from Messina and Giacobini (2013). 


\subsubsection{Class 3 semaphorins}

The class 3 semaphorins are produced as secreted proteins and consist of seven subgroups (3A-3G). Adams et al. (1997) verified that the initially synthesized pro-form undergoes proteolytic processing by furin or furin-like endoproteases generating functionally different isoforms. They pointed out that the consensus sequences for furindependent processing are highly conserved in all class 3 semaphorin proteins indicating a similar mechanism of regulation for all secreted semaphorins (Adams et al., 1997). For semaphorin 3C (SEMA3C), the proteolytic processing also induced by the matrix metalloprotease ADAMTS1 (ADAM with thrombospondin motif 1 ), resulting in a promoted cell migration in contrast to other class 3 semaphorins, was proved by Esselens et al. (2010). Adams et al. (1997) assumed that the activity of all secreted class 3 semaphorins depends on the extent of their proteolytic processing. Furthermore, dimerization of class 3 semaphorins were identified (Klostermann et al., 1998; Koppel and Raper, 1998). The existence of monomeric, dimeric and processed isoforms were proved for semaphorin $3 \mathrm{E}$ (SEMA3E) (Christensen et al., 2005).

\subsubsection{SEMA3A mutations contribute to the phenotype of Kallmann syndrome}

The involvement of semaphorin 3A (SEMA3A) in the navigation of primary olfactory axons during embryogenesis contributes to the phenotype of Kallmann syndrome (MIM 147950) (Hanchate et al., 2012; Young et al., 2012). This genetically heterogeneous congenital disorder is characterized by hypogonadotropic hypogonadism and an impaired sense of smell (Dodé and Hardelin, 2009). SEMA3A mutations were identified in Kallmann syndrome patients (Hanchate et al., 2012). Nevertheless, it was suggested that one heterozygous SEMA3A mutation alone cannot cause the symptoms, while a combination of a SEMA3A mutation with other gene mutations (digenic inheritance) can lead to the disease phenotype (Hanchate et al., 2012). Mutations in the Chd7 gene can be found in a minority of Kallmann syndrome patients (Kim et al., 2008; Bergman et al., 2011) and for this reason it was assumed that Kallmann syndrome represents the mild end of the phenotypic spectrum of CHARGE syndrome (Jongmans et al., 2009). Interestingly, Schulz et al. (2014b) confirmed an regulatory loop between Chd7 and Sema3a in mouse and Xenopus laevis. 


\section{$1.5 \quad$ Aim of the present study}

It was shown that $\mathrm{CHD7}$, the gene mutated in CHARGE syndrome, regulates genes which are involved in NCC formation and guidance as well as in interactions between NCCs and other tissues (Schulz et al., 2014b). Furthermore, it was assumed that SEMA3A mutations contribute to the pathogenesis of CHARGE syndrome (Schulz et al., 2014b).

The aim of the present study was to elucidate the pathomechanism behind the neurocristopathy CHARGE syndrome and especially the role of $S E M A 3 A$ in the pathogenesis of this complex malformation disorder.

\section{Establishment of an NCC model}

- To provide a suitable cell culture model for the analysis of NCCs in vitro, two different mouse NCC lines (a truncal and a cranial) should be tested for different marker genes using RT-PCR analysis in order to confirm the status of the NCCs.

- In both the truncal and the cranial NCC line the expression of Chd7 should be analyzed by RT-PCR and western blot analysis.

- The expression of the main class 3 semaphorin receptors, namely plexins and neuropilins, should be verified in NCCs using RT-PCR analysis.

\section{Role of SEMA3A in the pathogenesis of CHARGE syndrome}

- CHARGE syndrome patients with a pathogenic CHD7 mutation should be screened for additional mutations in the SEMA3A gene by sequencing analysis.

- The effect of the identified SEMA3A mutations should be investigated in vitro. Therefore, plasmids with the wildtype SEMA3A and the identified SEMA3A mutations should be generated. By western blot analysis, the wildtype and the variants of SEMA3A should be analyzed using a human cell culture system. Interactions between CHD7 and the wildtype SEMA3A as well as the SEMA3A variants should be identified with co-immunoprecipitation experiments and should be further examined using yeast two-hybrid assay. 


\section{$2 \quad$ Materials and Methods}

\section{$2.1 \quad$ Materials}

\subsubsection{Technical equipment}

\begin{tabular}{|c|c|}
\hline Technical equipment & Manufacturer \\
\hline 6-Tube Magnetic Separation Rack & New England Biolabs, Frankfurt/M \\
\hline 2720 Thermal Cycler & Applied Biosystems, Darmstadt \\
\hline 3500xL Genetic Analyzer & Applied Biosystems, Darmstadt \\
\hline 7900HT Fast Real-Time PCR System & Applied Biosystems, Darmstadt \\
\hline Accu-jet ${ }^{\circledR}$ & Brand, Wertheim \\
\hline Arium $^{\circledast} 611$ & Sartorius, Göttingen \\
\hline \multicolumn{2}{|l|}{ Autoclaves } \\
\hline DX-150 & Systec, Linden \\
\hline $\mathrm{FVS} / 2$ & Fedegari, Bedano (Suisse) \\
\hline VX-95 & Systec, Linden \\
\hline Balance CP3202S & Sartorius, Göttingen \\
\hline Camera (gel electrophoresis documentation) & Vilbert Lourmat, Eberhardzell \\
\hline \multicolumn{2}{|l|}{ Centrifuges } \\
\hline $4 \mathrm{~K} 15$ & Sigma, Osterode \\
\hline Heraeus Biofuge $^{\mathrm{TM}}$ Primo & Thermo Electron LED, Osterode \\
\hline Heraeus Fresco ${ }^{\mathrm{TM}} 21$ & Thermo Electron LED, Osterode \\
\hline Micro Centrifuge IR & Carl Roth, Karlsruhe \\
\hline Microfuge 1-15 & Sigma, Osterode \\
\hline Heraeus Multifuge X3R & Thermo Electron LED, Osterode \\
\hline Dry cabinet & Memmert, Schwabach \\
\hline Electrophorese chamber & Central workshop, University of Göttingen \\
\hline FluorChem ${ }^{\circledR} \mathrm{Q}$ & Alpha Innotech, Logan (USA) \\
\hline \multicolumn{2}{|l|}{ Freezers } \\
\hline$-20^{\circ} \mathrm{C}$ & Liebherr, Ochsenhausen \\
\hline$-80^{\circ} \mathrm{C}$ & Sanyo, Moriguchi (Japan) \\
\hline Herasafe $^{\mathrm{TM}}$ (biological safety cabinet) & Thermo Electron LED, Langenselbold \\
\hline \multicolumn{2}{|l|}{ Incubators } \\
\hline for bacteria $\left(37^{\circ} \mathrm{C}\right)$ & Memmert, Schwabach \\
\hline for cell culture $\left(37^{\circ} \mathrm{C}\right)$ & Sanyo, Munich \\
\hline for yeast $\left(30^{\circ} \mathrm{C}\right)$ & Memmert, Schwabach \\
\hline Innova ${ }^{\circledR} 40$ (incubation shaker) & New Brunswick Scientific, Nürtingen \\
\hline Laboport $^{\circledR}$-Membrane-Vacuumpump N86KN.18 & KNF Neuberger, Freiburg \\
\hline
\end{tabular}


Magnetic stirrer

Microscopes

confocal laser scanning microscope IX81

inverted routine microscope Primovert

stereo microscope Stemi SV 11

Microwave OMW 310-S

Mini Protean ${ }^{\circledR}$ II Cell

NanoDrop 2000c

Novex ${ }^{\circledR}$ Mini-Cell Electrophoresis System

pH-Meter PB-11

Pipettes

Multipette ${ }^{\circledast}$ M4

Pipetman

Research

Transferpette ${ }^{\circledR}-8$

Power supplies

Apelex PS 304 minipac II

Power PAC 3000

Printer system (gel electrophoresis documentation)

Doc print ${ }^{\mathrm{TM}} \mathrm{VX} 2$

P95

Refrigerators

Roll shaker RM5 Assistent 348

Scissors

HSB 006-10

HSB 530-08

Semi-Automatic Sheet Heat Sealer

Shaker

Steel bead, stainless (ø $5 \mathrm{~mm}$ )

Sterilizer

Stuart ${ }^{\circledR}$ SB2 rotator

Synergy ${ }^{\mathrm{TM}} \mathrm{Mx}$ plate reader

Thermomixer 5436 / compact

TissueLyser LT

Tweezer HWC 110-10

UVsolo TS Imaging System

UV table ECX-F20.M

Vortex-Genie ${ }^{\circledR} 2$

Water bath
IKA Labortechnik, Staufen

Olympus, Hamburg

Zeiss, Göttingen

Zeiss, Göttingen

ok. / Venalisia Import, Ingolstadt

Bio-Rad, Munich

Peqlab, Erlangen

Invitrogen, Karlsruhe

Sartorius, Göttingen

Eppendorf, Hamburg

Gilson, Limburg-Offheim

Eppendorf, Hamburg

Brand, Wertheim

Biostep, Jahnsdorf

Bio-Rad, Munich

Peqlab, Erlangen

Mitsubishi Electric, Tokyo (Japan)

Liebherr, Ochsenhausen

Bosch, Stuttgart

Karl Hecht, Sondheim

Hammacher, Solingen

Hammacher, Solingen

4titude, Berlin

IKA Labortechnik, Staufen

Qiagen, Hilden

Memmert, Schwabach

Bibby Scientific, Staffordshire (UK)

BioTek, Friedrichshall

Eppendorf, Hamburg

Qiagen, Hilden

Hammacher, Solingen

Biometra, Göttingen

PeqLab, Erlangen

Scientific Industries, New York (USA)

GFL, Großburgwedel 


\subsubsection{Consumable materials}

\begin{tabular}{|c|c|}
\hline Product name & Manufacturer \\
\hline Blotting Paper Sheets & Sartorius, Göttingen \\
\hline \multicolumn{2}{|l|}{ Cell culture flasks } \\
\hline $25 \mathrm{~cm}^{2}, 75 \mathrm{~cm}^{2}$ with filter & Sarstedt, Nümbrecht \\
\hline $75 \mathrm{~cm}^{2}$ without filter & Greiner Bio-One, Frickenhausen \\
\hline $300 \mathrm{~cm}^{2}$ with filter & TPP, Trasadingen (Switzerland) \\
\hline \multicolumn{2}{|l|}{ Cell culture plates } \\
\hline 96-well & Nuclon A/S, Roskilde (Danmark) \\
\hline 24-well & Th. Geyer, Renningen \\
\hline 12-well & Greiner Bio-One, Frickenhausen \\
\hline 6-well & Greiner Bio-One, Frickenhausen \\
\hline $10 \mathrm{~cm} \varnothing$ & Nuclon A/S, Roskilde (Danmark) \\
\hline Glas bottles & Schott, Mainz / Biochrom, Berlin \\
\hline Combitips (advanced) & Eppendorf, Hamburg \\
\hline Counting chamber, bright-lined (Neubauer) & Sarstedt, Nümbrecht \\
\hline Cover glasses (24 x $60 \mathrm{~mm})$ & Menzel Gläser, Braunschweig \\
\hline Cryovials & Greiner Bio-One, Frickenhausen \\
\hline Culture slides (8-well) & BD Biosciences, Heidelberg \\
\hline Cuvettes, $10 \mathrm{~mm}$ & Sarstedt, Nümbrecht \\
\hline Disposable syringes & BD Biosciences, Heidelberg \\
\hline Filter $(\varnothing 0.20 \mu \mathrm{m}, \varnothing 0.45 \mu \mathrm{m})$ & Th. Geyer, Renningen \\
\hline Gas cartouche (propane/butane) & Th. Geyer, Renningen \\
\hline Glass pipettes & Schütt, Göttingen \\
\hline Gloves & Lab Logistic Group, Meckenheim \\
\hline Amersham $^{\mathrm{TM}}$ Protran $^{\mathrm{TM}}$ Supported $0.45 \mu \mathrm{m}$ NC & GE Healthcare, Freiburg \\
\hline $\begin{array}{l}\text { Amersham }{ }^{\mathrm{TM}} \text { Hybond }^{\mathrm{TM}} \text {-P PVDF Transfer Membrane } \\
\quad 0.45 \mu \mathrm{m}\end{array}$ & GE Healthcare, Freiburg \\
\hline Mr. Frosty ${ }^{\mathrm{TM}}$ Freezing Container & Kisker, Steinfurt \\
\hline MultiScreen ${ }^{\circledast}$ PCR96 Filter Plates & Merck Millipore, Darmstadt \\
\hline NuPAGE $^{\circledR} 3-8 \%$ Tris-Acetate Gels, $1.0 \mathrm{~mm}$, 10-well & Invitrogen, Karlsruhe \\
\hline NuPAGE ${ }^{\circledR} 4-12 \%$ Bis-Tris Gels, 1.0 mm, 10-well & Invitrogen, Karlsruhe \\
\hline NuPAGE ${ }^{\circledR} 4-12 \%$ Bis-Tris Midi Gels, 1.0 mm, 20-well & Invitrogen, Karlsruhe \\
\hline Pasteur pipettes & Th. Geyer, Renningen \\
\hline PCR cups $(0.2 \mathrm{ml})$ & Sarstedt, Nümbrecht \\
\hline Petri dishes & Sarstedt, Nümbrecht \\
\hline Pipette tips (without filter) & Glasgerätebau Ochs, Bovenden \\
\hline Pipette tips (with filter) & Kisker, Steinfurt \\
\hline qRT-PCR plates (384-well) & 4titude, Berlin \\
\hline
\end{tabular}


qRT-PCR Adhesive Clear Seals

Reaction tubes $(1.5 \mathrm{ml}, 2 \mathrm{ml})$

Scalpel

Serological pipettes

$2 \mathrm{ml}, 5 \mathrm{ml}, 10 \mathrm{ml}$

$25 \mathrm{ml}$

Transfection tubes

Transfer pipettes (3.5 ml)

Tubes

$13 \mathrm{ml}$

$15 \mathrm{ml}, 50 \mathrm{ml}$

Vivaspin $^{\circledR}$ Turbo 15 (30 kDa MWCO) 4titude, Berlin

Glasgerätebau Ochs, Bovenden

Pfm medical, Cologne

Sarstedt, Nümbrecht

Greiner Bio-One, Frickenhausen

Sarstedt, Nümbrecht

Sarstedt, Nümbrecht

Sarstedt, Nümbrecht

Greiner Bio-One, Frickenhausen

Sartorius, Göttingen

\subsubsection{Chemicals, biochemicals and reagents}

\begin{tabular}{|c|c|}
\hline Chemical / Biochemical / Reagent & Manufacturer \\
\hline 1-Thioglycerol & Sigma-Aldrich, Deisenhofen \\
\hline 1,4-Dithiothreitol (DTT) & AppliChem, Darmstadt \\
\hline 4-Hydroxytamoxifen (4-OHT) & Sigma-Aldrich, Deisenhofen \\
\hline Acetic acid & Merck, Darmstadt \\
\hline Adenine hemisulfate salt & Sigma-Aldrich, Deisenhofen \\
\hline Agar-Agar, Kobe I & Carl Roth, Karlsruhe \\
\hline Agarose & Peqlab, Erlangen \\
\hline Ammonium sulfate & Carl Roth, Karlsruhe \\
\hline Ampicillin sodium salt & Carl Roth, Karlsruhe \\
\hline Ampuwa $^{\circledR}$ & Fresenius, Bad Homburg \\
\hline B-27 ${ }^{\oplus}$ Supplement (50x) & Gibco, Eggenstein \\
\hline Barricidal $^{\circledR}$ & Interchem Hygiene, Zürich (Switzerland) \\
\hline Boric acid & ICN Biomedicals, Eschwege \\
\hline Bovine serum albumin (BSA) Fraction $V$ & PAA Laboratories, Linz (Austria) \\
\hline Bovine Serum Albumin Standard Ampules (2 mg/ml) & Thermo Scientific, Rockford (USA) \\
\hline Bromphenolblue & Carl Roth, Karlsruhe \\
\hline Calcium chloride $\left(\mathrm{CaCl}_{2}\right)$ & Carl Roth, Karlsruhe \\
\hline Chloroform & J.T. Baker, Griesheim \\
\hline Chicken embryo extract (CEE) & Dr. Sven Lindner, Ruhr-University Bochum \\
\hline Complete ES Cell Medium with 15\% FBS and LIF & Merck Millipore, Darmstadt \\
\hline cOmplete ${ }^{\mathrm{TM}}$ Protease Inhibitor Cocktail Tablets & Roche, Penzberg \\
\hline Cryo-SFM & PromoCell, Heidelberg \\
\hline
\end{tabular}


$\mathrm{D}(+)$-Glucose

Deoxynucleotide triphosphates (dNTPs)

Diethyl pyrocarbonate (DEPC)

Dimethyl sulfoxide (DMSO)

DirectPCR ${ }^{\circledast}$ Lysis Reagent Tail

Disodium hydrogen phosphate $\left(\mathrm{Na}_{2} \mathrm{HPO}_{4}\right)$

DO Supplement (-Ade/-His/-Leu/-Trp)

Dulbecco's Modified Eagle Medium (DMEM)

Dulbecco's Modified Eagle Medium/Nutrient Mixture F-12 (DMEM/F-12)

Dulbecco's Phosphate-Buffered Saline (DPBS)

Epidermal growth factor, human (EGF)

Ethanol

Ethidium bromide

Ethylenediaminetetraacetic acid disodium salt dihydrate (EDTA)

Fetal bovine serum, SeraPlus (FBS)

Fibroblast growth factor 2, human (FGF-2)

Fibronectin, human (FC010)

Fluoroshield $^{\mathrm{TM}}$ with Dapi

Glycerol

Glycine

Halt $^{\mathrm{TM}}$ Protease Inhibitor Single Use Cocktail, EDTAfree $(100 x)$

Hydrochloric acid $(\mathrm{HCl})$

Immersol $^{\mathrm{TM}} 518 \mathrm{~F}$

Isopropanol

Kanamycin sulfate

L-Histidine monohydrochloride monohydrate

Lipofectamine $^{\circledR} 2000$ Transfection Reagent

Lithium acetate (LiAc)

Matrige ${ }^{\circledR}$ Basement Membrane Matrix

Methanol

Milk powder

MEM Non-Essential Amino Acids (NEAA)

N-2 Supplement (100x)

NuPAGE ${ }^{\circledR}$ LDS Sample Buffer (4x)

NuPAGE ${ }^{\oplus}$ MES SDS Running Buffer (20x)

NuPAGE ${ }^{\circledR}$ Tris-Acetate SDS Running Buffer (20x)
Carl Roth, Karlsruhe

Bio-Budget Technologies, Krefeld

Carl Roth, Karlsruhe

Carl Roth, Karlsruhe

Peqlab, Erlangen

Carl Roth, Karlsruhe

Clontech, Heidelberg

PAN-Biotech, Aidenbach

Gibco, Eggenstein

PAN-Biotech, Aidenbach

Sigma-Aldrich, Deisenhofen

J.T. Baker, Griesheim

Inno-Train Diagnostik, Kronberg/Ts.

MP Biomedicals, Eschwege

PAN-Biotech, Aidenbach

Merck Millipore, Darmstadt

PeproTech, Hamburg

Merck Millipore, Darmstadt

Sigma-Aldrich, Deisenhofen

Carl Roth, Karlsruhe

Carl Roth, Karlsruhe

Thermo Scientific, Rockford (USA)

J.T. Baker, Griesheim

Zeiss, Oberkochen

J.T. Baker, Griesheim

Carl Roth, Karlsruhe

Sigma-Aldrich, Deisenhofen

Invitrogen, Karlsruhe

Carl Roth, Karlsruhe

Corning, Wiesbaden

J.T. Baker, Griesheim

Carl Roth, Karlsruhe

Gibco, Eggenstein

Gibco, Eggenstein

Invitrogen, Karlsruhe

Invitrogen, Karlsruhe

Invitrogen, Karlsruhe 
Opti-MEM $^{\circledast}$ I Reduced Serum Medium

P1 / P2 / P3 Buffer

Paraformaldehyde (PFA)

Penicillin-Streptomycin

Peptone ex casein

Polyethylene glycol 4000 (PEG4000)

Potassium chloride $(\mathrm{KCl})$

Potassium dihydrogen phosphate $\left(\mathrm{KH}_{2} \mathrm{PO}_{4}\right)$

Proteinase $\mathrm{K}$

Protein G Magnetic Beads

Random Hexamers $(50 \mu \mathrm{M})$

Restriction endonucleases

Roti $^{\circledR}$-Nanoquant

Roti $^{\circledR}$-Safe

S.O.C. medium

Sodium acetate

Sodium chloride $(\mathrm{NaCl})$

Sodium hydroxide $(\mathrm{NaOH})$

Streptomycin sulfate salt

TE buffer

Thimerosal

Tris(hydroxymethyl)aminomethane (Tris)

Triton X-100

Trizol Reagent

TrypLE ${ }^{\text {TM }}$ Express

Trypsin-EDTA

Trypsin Inhibitor, from Glycine max (soy-bean)

Tunicamycin from Streptomyces sp.

Tween ${ }^{\circledR} 20$

Western Blocking Reagent

Yeast extract

Yeastmaker ${ }^{\mathrm{TM}}$ Carrier DNA

Yeast Nitrogen Base Without Amino Acids and Ammonium Sulfate (YNB)
Gibco, Eggenstein

Qiagen, Hilden

Carl Roth, Karlsruhe

Gibco, Eggenstein

Carl Roth, Karlsruhe

Carl Roth, Karlsruhe

Carl Roth, Karlsruhe

Carl Roth, Karlsruhe

Carl Roth, Karlsruhe

New England Biolabs, Frankfurt/M

Invitrogen, Karlsruhe

Invitrogen, Karlsruhe

New England Biolabs, Frankfurt/M

Carl Roth, Karlsruhe

Carl Roth, Karlsruhe

Invitrogen, Karlsruhe

Carl Roth, Karlsruhe

AppliChem, Darmstadt

J.T. Baker, Griesheim

Sigma-Aldrich, Deisenhofen

Invitrogen, Karlsruhe

Sigma-Aldrich, Deisenhofen

AppliChem, Darmstadt

Serva, Heidelberg

Invitrogen, Karlsruhe

Gibco, Eggenstein

PAN-Biotech, Aidenbach

Sigma-Aldrich, Deisenhofen

Sigma-Aldrich, Deisenhofen

Carl Roth, Karlsruhe

Sigma-Aldrich, Deisenhofen

Carl Roth, Karlsruhe

Clontech, Saint-Germain-en-Laye (France)

Sigma-Aldrich, Deisenhofen 


\subsubsection{Ready-to-use reaction systems}

\begin{tabular}{|c|c|}
\hline Reaction system & Manufacturer \\
\hline BigDye $^{\circledR}$ Terminator v1.1 Cycle Sequencing Kit & Applied Biosystems, Darmstadt \\
\hline DNase I Kit & Sigma-Aldrich, Deisenhofen \\
\hline HiPure Plasmid Midiprep Kit & Invitrogen, Karlsruhe \\
\hline HotStarTaq $^{\circledR}$ Plus Master Mix Kit & Qiagen, Hilden \\
\hline $\begin{array}{l}\text { Immobilon }^{\mathrm{TM}} \text { Western Chemiluminescent HRP } \\
\text { Substrate }\end{array}$ & Merck Millipore, Darmstadt \\
\hline Immolase ${ }^{\mathrm{TM}}$ DNA Polymerase & Bioline, Luckenwalde \\
\hline In-Fusion ${ }^{\circledR} \mathrm{HD}$ Cloning Kit & Clontech, Saint-Germain-en-Laye (France) \\
\hline MSB $^{\circledR}$ Spin PCRapace Kit & Invitek, Berlin \\
\hline $\begin{array}{l}\text { NE-PER }{ }^{\circledR} \text { Nuclear and Cytoplasmic Extraction } \\
\text { Reagents Kit }\end{array}$ & Thermo Scientific, Rockford (USA) \\
\hline PfuUltra ${ }^{\circledast}$ High-Fidelity DNA Polymerase & Agilent Technologies, Waldbronn \\
\hline Platinum ${ }^{\circledR}$ Taq DNA Polymerase & Invitrogen, Karlsruhe \\
\hline Q5 ${ }^{\circledR}$ High-Fidelity DNA Polymerase & New England Biolabs, Frankfurt/M \\
\hline QIAquick $^{\circledR}$ Gel Extraction Kit & Qiagen, Hilden \\
\hline QuantiTect $^{\circledR}$ SYBR $^{\circledR}$ Green PCR Kit & Qiagen, Hilden \\
\hline QuikChange II XL Site-Directed Mutagenesis Kit & Agilent Technologies, Waldbronn \\
\hline Superscript ${ }^{\mathrm{TM}}$ II Reverse Transcriptase Kit & Invitrogen, Karlsruhe \\
\hline
\end{tabular}

\subsubsection{Sterilization}

If not indicated differently, buffers and solutions were sterilized in a vapor autoclave for $20 \mathrm{~min}$ at $120^{\circ} \mathrm{C}$ and $10^{5} \mathrm{~Pa}$. Heat sensitive solutions were sterile filtrated using filtration units of $0.20-0.45 \mu \mathrm{m}$ pore size. Laboratory equipment were either autoclaved as mentioned above or heat sterilized at $180^{\circ} \mathrm{C}$ overnight.

\subsubsection{Buffers and solutions}

\begin{tabular}{l|l}
\hline Buffer / Solution & Composition \\
\hline 4-OHT solution & $5.16 \mathrm{mM} 4-\mathrm{OHT}$ in Ethanol \\
Adenine solution & $10 \mathrm{mg} / \mathrm{ml}$ Adenine in Ampuwa \\
Ampicillin solution & $50 \mathrm{mg} / \mathrm{ml}$ Ampicillin in Ampuwa
\end{tabular}


Antibody diluent solution

(Immunofluorescence staining)

Antibody diluent solution I

(Western blot analysis)

Antibody diluent solution II

(Western blot analysis)

Blocking solution

(Immunofluorescence staining)

Blocking solution I

(Western blot analysis)

Blocking solution II

(Western blot analysis)

Casein solution (10\%)

dNTPs solution

DPBS with $0.1 \%$ Tween 20 (DPBST)

EGF (human) solution

DEPC- $\mathrm{H}_{2} \mathrm{O}$

FGF-2 (human) solution
$1 \%$ BSA in DPBST

$1 \%$ BSA or $2 \%$ milk powder in TBST

$0.1 \%$ Casein solution in TBST

300 mM Glycine

$3 \%$ BSA

$0.1 \%$ Tween 20

in PBS

$5 \%$ BSA or milk powder in TBST

$0.1 \%$ Casein solution in TBST

Western Blocking Reagent (with 10\% Casein)

$1 \%$ Thimerosal

$10 \mathrm{mM}$ dATP

$10 \mathrm{mM}$ dCTP

$10 \mathrm{mM}$ dGTP

$10 \mathrm{mM}$ dTTP

in Ampuwa

$0.1 \%$ Tween 20 in DPBS

$0.25 \mathrm{mg} / \mathrm{ml}$ EGF in $0.1 \% \mathrm{BSA} / 10 \mathrm{mM}$ Acetic acid

$0.1 \%$ Diethylpyrocarbonate in $\mathrm{ddH}_{2} \mathrm{O}$

Incubation for $24 \mathrm{~h}$ by stirring

$25 \mu \mathrm{g} / \mathrm{ml}$ FGF-2 (Merck Millipore) in $1 \mathrm{mM}$

1-Thioglycerol / $0.01 \mathrm{mM}$ Tris, $\mathrm{pH} 7.0$

$0.1 \mathrm{mg} / \mathrm{ml}$ FGF-2 (PeproTech) in 0.1\% BSA/PBS 
Fibronectin (human) solution

Glucose solution (40\%)

Glycerol solution (50\%)

Histidine solution

Kanamycin solution

LiAc solution (10x)

Loading buffer

(Agarose gel electrophoresis)

Lysis buffer

PBS buffer (10x)

PEG 4000 solution (50\%)
$50 \mu \mathrm{g} / \mathrm{ml}$ Fibronectin in PBS

$40 \%$ Glucose in $\mathrm{dd}_{2} \mathrm{O}$

autoclave for $15 \mathrm{~min}$ at $121^{\circ} \mathrm{C}$

$50 \%$ Glycerol in dd $\mathrm{H}_{2} \mathrm{O}$

$10 \mathrm{mg} / \mathrm{ml}$ Histidine in Ampuwa

50 mg/ml Kanamycin in Ampuwa

$100 \mathrm{mM} \mathrm{LiAc}$ in $\mathrm{ddH}_{2} \mathrm{O}$

adjust to $\mathrm{pH} 7.5$

autoclave for $15 \mathrm{~min}$ at $121^{\circ} \mathrm{C}$

0.1 M EDTA

$50 \%$ Glycerol

$0.02 \%$ Xylene cyanol

$0.02 \%$ Bromphenol blue

$0.02 \%$ SDS

in Ampuwa

$300 \mathrm{mM} \mathrm{NaCl}$

$50 \mathrm{mM}$ Tris- $\mathrm{HCl}, \mathrm{pH} 7.5$

5 mM EDTA

$0.1 \%$ Triton $X-100$

Protease inhibitor cocktail tablet

in $\mathrm{ddH}_{2} \mathrm{O}$

$1.4 \mathrm{M} \mathrm{NaCl}$

$90 \mathrm{mM} \mathrm{Na}_{2} \mathrm{HPO}_{4}$

$27 \mathrm{mM} \mathrm{KCl}$

$15 \mathrm{mM} \mathrm{KH}_{2} \mathrm{PO}_{4}$

in $\mathrm{ddH}_{2} \mathrm{O}$

adjust to $\mathrm{pH} 7.4$

$50 \%$ PEG 4000 in dd $_{2} \mathrm{O}$

autoclave for $15 \mathrm{~min}$ at $121^{\circ} \mathrm{C}$ 


\begin{tabular}{|c|c|}
\hline PEG/LiAc solution & $\mid \begin{array}{l}1 \text { ml TE buffer }(10 x) \\
1 \text { ml LiAc solution (10x) } \\
8 \text { ml PEG } 4000 \text { solution }(50 \%)\end{array}$ \\
\hline PFA solution (4\%) & $\begin{array}{l}\text { 4\% PFA in PBS } \\
\text { adjust to } \mathrm{pH} 6-7\end{array}$ \\
\hline Proteinase $\mathrm{K}$ solution & $10 \mathrm{mg} / \mathrm{ml}$ Proteinase $\mathrm{K}$ in Ampuwa \\
\hline TBE buffer $(5 x)$ & $\begin{array}{l}445 \mathrm{mM} \text { Tris } \\
445 \mathrm{mM} \text { Boric acid } \\
0.01 \mathrm{M} \text { EDTA } \\
\text { in } \mathrm{ddH}_{2} \mathrm{O}\end{array}$ \\
\hline TBS buffer & $\begin{array}{l}154 \mathrm{mM} \mathrm{NaCl} \\
10 \mathrm{mM} \text { Tris- } \mathrm{HCl}, \mathrm{pH} 7.5 \\
\text { in } \mathrm{ddH}_{2} \mathrm{O}\end{array}$ \\
\hline TBST buffer & $\begin{array}{l}154 \mathrm{mM} \mathrm{NaCl} \\
10 \mathrm{mM} \text { Tris- } \mathrm{HCl}, \mathrm{pH} 7.5 \\
0.1 \% \text { Tween } 20 \\
\text { in } \mathrm{ddH}_{2} \mathrm{O}\end{array}$ \\
\hline TE buffer (10x) & $\begin{array}{l}10 \% 1 \mathrm{M} \text { Tris- } \mathrm{HCl}, \mathrm{pH} 7.5 \\
2 \% 0.5 \mathrm{M} \text { EDTA, } \mathrm{pH} 8.0 \\
\text { in } \mathrm{ddH}_{2} \mathrm{O} \\
\text { autoclave for } 15 \mathrm{~min} \text { at } 121^{\circ} \mathrm{C}\end{array}$ \\
\hline TE/LiAc solution & $\begin{array}{l}500 \mu \mathrm{l} \text { TE buffer }(10 x) \\
500 \mu \mathrm{l} \text { LiAc solution }(10 \mathrm{x}) \\
4 \mathrm{ml} \text { Ampuwa }\end{array}$ \\
\hline $\begin{array}{l}\text { Transfer buffer (10x, without Methanol) } \\
\text { (Western blot analysis) }\end{array}$ & $\begin{array}{l}1.92 \mathrm{M} \text { Glycine } \\
250 \mathrm{mM} \text { Tris } \\
\text { in } \mathrm{ddH}_{2} \mathrm{O}\end{array}$ \\
\hline $\begin{array}{l}\text { Transfer buffer I } \\
\text { (Western blot analysis) }\end{array}$ & $\begin{array}{l}8 \% \text { Transfer buffer (10x, without Methanol) } \\
20 \% \text { Methanol } \\
\text { in } \mathrm{ddH}_{2} \mathrm{O}\end{array}$ \\
\hline
\end{tabular}


Transfer buffer II

(Western blot analysis)

192 mM Glycine

$25 \mathrm{mM}$ Tris

$10 \%$ Methanol

$0.025 \%$ SDS

in $\mathrm{ddH}_{2} \mathrm{O}$

adjust to $\mathrm{pH} 8.3$

Tris/HCl buffer

$1 \mathrm{M}$ Tris in $\mathrm{ddd}_{2} \mathrm{O}$

adjust with $\mathrm{HCl}$ to $\mathrm{pH} 7.5$

Triton X-100 solution (0.1\%)

$0.1 \%$ Triton $X-100$ in DPBS

\subsubsection{Plasmids and vectors}

\begin{tabular}{l|l}
\hline Plasmid / Vector & Source \\
\hline CHD7-2-pGBKT7 & Schulz et al. (2014a) \\
CHD7-3-pGBKT7 & Schulz et al. (2014a) \\
CHD7-4-pGBKT7 & Schulz et al. (2014a) \\
CHD7-Cr1-3-pGBKT7 & Batsukh et al. (2010) \\
CHD8-pGADT7-Rec & Batsukh et al. (2010) \\
RbBP5-pCMV-HA & Schulz et al. (2014a) \\
SEMA3A WT-pcDNA3.1/myc-His B & AG Pauli, Institute of Human Genetics (Göttingen) \\
SEMA3A WT-pCMV-HA & generated in the present thesis (chapter 3.7) \\
SEMA3A c.196C>T/p.R66W-pCMV-HA & generated in the present thesis (chapter 3.7) \\
SEMA3A c.2002A>G/p.I668V-pCMV-HA & generated in the present thesis (chapter 3.7) \\
SEMA3A WT-pGADT7 & generated in the present thesis (chapter 3.7) \\
SEMA3A c.196C>T/p.R66W-pGADT7 & generated in the present thesis (chapter 3.7) \\
SEMA3A c.2002A>G/p.I668V-pGADT7 & generated in the present thesis (chapter 3.7) \\
pcDNA ${ }^{\text {TM} 3.1 / m y c-H i s ~ B ~}$ & Invitrogen, Karlsruhe \\
pCMV-HA & Clontech, Saint-Germain-en-Laye (France) \\
pGADT7 & Clontech, Saint-Germain-en-Laye (France) \\
pGBKT7 & Clontech, Saint-Germain-en-Laye (France)
\end{tabular}




\subsubsection{Bacterial strains}

\begin{tabular}{|c|c|}
\hline Bacterial strain & Source \\
\hline $\begin{array}{ccc}\text { Escherichia coli } & \text { DH5a competent cells } \\
\text { (Subcloning } & \text { Efficiency }^{\mathrm{TM}} & \mathrm{DH} 5 \alpha^{\mathrm{TM}}, \\
\text { Chemically Competent Cells) }\end{array}$ & Invitrogen, Karlsruhe \\
\hline
\end{tabular}

\subsubsection{Media and agar plates for bacterial culture}

\begin{tabular}{|c|c|}
\hline Medium / Plate & Composition \\
\hline LB medium & $\begin{array}{l}1 \% \text { Peptone ex casein } \\
1 \% \mathrm{NaCl} \\
0.5 \% \text { Yeast extract } \\
\text { in } \mathrm{ddH}_{2} \mathrm{O}\end{array}$ \\
\hline LB/Ampicillin & $0.1 \% \mathrm{mg} / \mathrm{ml}$ Ampicillin in LB Medium \\
\hline LB/Kanamycin & $0.1 \% \mathrm{mg} / \mathrm{ml}$ Kanamycin in LB Medium \\
\hline LB agar plates & $\begin{array}{l}1 \% \text { Peptone ex casein } \\
1 \% \mathrm{NaCl} \\
0.5 \% \text { Yeast extract } \\
1.5 \% \text { Agar-Agar } \\
\text { in } \mathrm{ddH}_{2} \mathrm{O}\end{array}$ \\
\hline
\end{tabular}

\subsubsection{Eukaryotic cell lines}

\begin{tabular}{l|l|l|l}
\hline Cell line & Description & Source & Culture medium \\
\hline HEK293 & $\begin{array}{l}\text { human embryonic kidney 293 } \\
\text { cells }\end{array}$ & $\begin{array}{l}\text { ATCC }{ }^{\oplus}\left(\mathrm{CRL}-1573^{\mathrm{TM}}\right) \\
\text { LGC Standards (Wesel) }\end{array}$ & FB medium \\
\hline JoMa1 & murine trunk NCC line & $\begin{array}{l}\text { Dr. Sven Lindner } \\
\text { (Ruhr-University Bochum) }\end{array}$ & NCC medium \\
\hline JoMa1.3 & $\begin{array}{l}\text { murine trunk NCC line (clonally } \\
\text { derived subline from JoMa1) }\end{array}$ & $\begin{array}{l}\text { Dr. Sven Lindner } \\
\text { (Ruhr-University Bochum) }\end{array}$ & NCC medium \\
\hline O9-1 & murine cranial NCC line & $\begin{array}{l}\text { Merck Millipore (SCC049) } \\
\text { (Darmstadt) }\end{array}$ & ESC medium \\
\hline
\end{tabular}




\subsubsection{Media for eukaryotic cell culture}

\begin{tabular}{|c|c|}
\hline Medium & Composition \\
\hline ESC medium & $\begin{array}{l}\text { Complete ES Cell Medium with FBS and LIF } \\
25 \mathrm{ng} / \mathrm{ml} \text { FGF-2 (human, PeproTech) }\end{array}$ \\
\hline $\begin{array}{l}\text { Fibroblast medium } \\
\text { (FB medium) }\end{array}$ & $\begin{array}{l}\text { DMEM } \\
10 \% \text { FBS } \\
1 \times \text { NEAA } \\
100 \mathrm{U} / \mathrm{ml} / 0.1 \mathrm{mg} / \mathrm{ml} \text { Penicillin/Streptomycin }\end{array}$ \\
\hline NCC medium & $\begin{array}{l}\text { DMEM/F-12 } \\
10 \% \text { CEE } \\
1 \times \text { B-27 Supplement } \\
1 \times \text { N-2 Supplement } \\
100 \mathrm{U} / \mathrm{ml} / 0.1 \mathrm{mg} / \mathrm{ml} \text { Penicillin/Streptomycin } \\
10 \mathrm{ng} / \mathrm{ml} \text { EGF (human) } \\
1 \mathrm{ng} / \mathrm{ml} \text { FGF-2 (human, Merck Millipore) } \\
200 \mathrm{nM} 4-\mathrm{OHT}\end{array}$ \\
\hline
\end{tabular}

\subsubsection{Freezing media}

\begin{tabular}{l|l}
\hline Medium & Composition \\
\hline ESC freezing medium & Cryo-SFM (ready to use) \\
Fibroblast freezing medium & $60 \%$ DMEM \\
(FB freezing medium) & $20 \%$ FBS \\
& $20 \%$ DMSO \\
NCC freezing medium & $90 \%$ Complete ES Cell Medium with FBS and LIF \\
& $10 \%$ DMSO
\end{tabular}

\subsubsection{Yeast strains}

\begin{tabular}{l|l}
\hline Yeast strain & Source \\
\hline Y2HGold & Clontech, Saint-Germain-en-Laye (France)
\end{tabular}




\subsubsection{Media and agar plates for yeast culture}

\begin{tabular}{|c|c|}
\hline Medium / Agar plate & Composition \\
\hline SD medium & 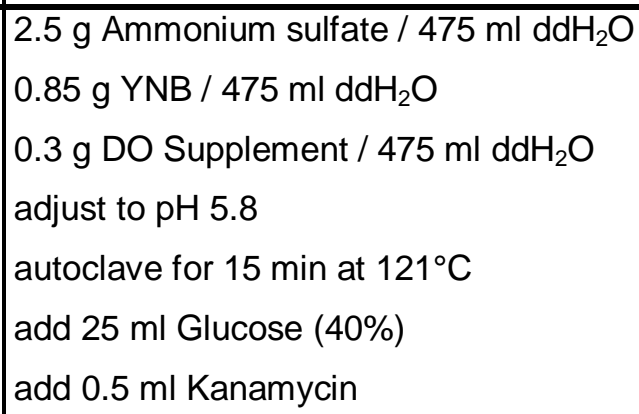 \\
\hline SD medium with Adenine and Histidine & 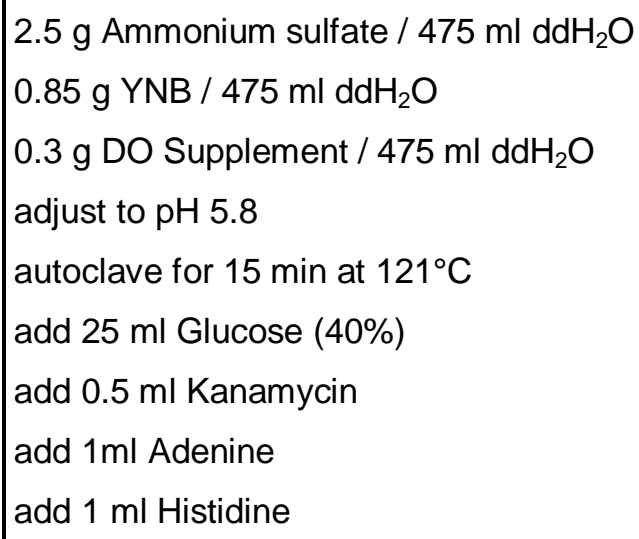 \\
\hline YPDA medium & 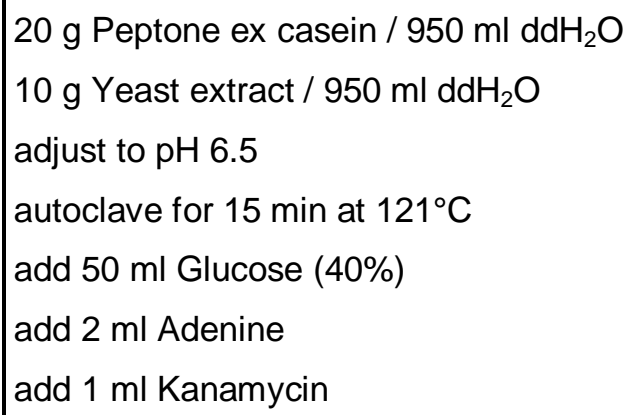 \\
\hline -LT agar plates & 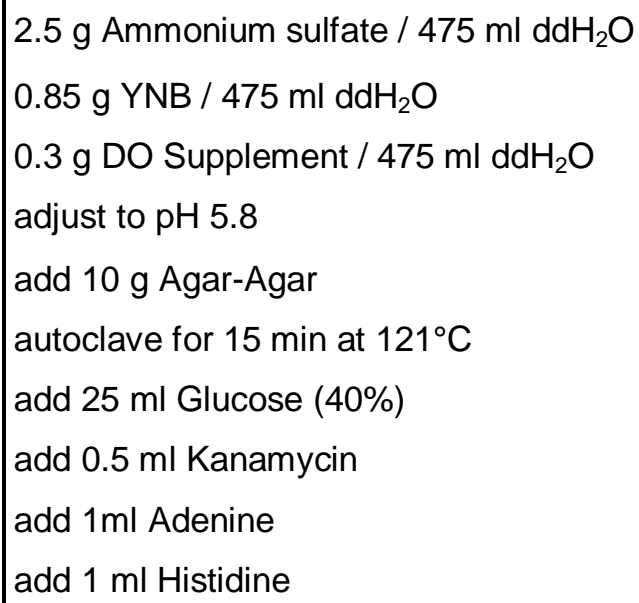 \\
\hline
\end{tabular}


- LTHA agar plates

YPDA agar plates



\subsubsection{Antibodies}

\subsubsection{Primary antibodies}

\begin{tabular}{l|l|l|l|l|l}
\hline Antigen & Company & Code & Species & Clonality & Dilution \\
\hline a-Tubulin & Sigma-Aldrich, Deisenhofen & T5168 & mouse & monoclonal & $\begin{array}{l}\text { ICC 1:10,000 } \\
\text { WB 1:4000 }\end{array}$ \\
\hline Baf47 & Abcam, Cambridge (UK) & ab126734 & rabbit & monoclonal & WB 1:1000 \\
\hline \multirow{2}{*}{ Chd7 } & Cell Signaling, Danvers (USA) & 6505 & rabbit & monoclonal & ICC 1:20 \\
\cline { 2 - 6 } & Abcam, Cambridge, (UK) & ab31824 & rabbit & polyclonal & WB 1:1000 \\
\hline HA & Sigma-Aldrich, Deisenhofen & 11867431001 & rat & monoclonal, & WB 1:1000 \\
& & & & $3 F 10$ & \\
\hline Sema3a & Abcam, Cambridge (UK) & ab23393 & rabbit & polyclonal & ICC 1:20 \\
& & & & WB 1:1000 \\
\hline
\end{tabular}




\subsubsection{Secondary antibodies}

\begin{tabular}{|c|c|c|c|}
\hline Antibody & Company & Code & Dilution \\
\hline $\begin{array}{l}\text { Anti-Rabbit lgG (whole molecule), } \\
F\left(a b^{\prime}\right)_{2} \text { fragment-Cy3 antibody produced } \\
\text { in sheep }\end{array}$ & Sigma-Aldrich, Deisenhofen & $\mathrm{C} 2306$ & ICC 1:500 \\
\hline $\begin{array}{l}\text { F(ab')2-Goat anti-Mouse lgG }(\mathrm{H}+\mathrm{L}) \\
\text { Cross-Adsorbed Secondary Antibody, } \\
\text { Alexa Fluor }{ }^{\circledR} 488\end{array}$ & Invitrogen, Karlsruhe & A11017 & ICC 1:500 \\
\hline Goat lgG anti-Mouse lgG (H+L)-HRPO & Dianova, Hamburg & $115-035-062$ & WB $1: 10,000$ \\
\hline Goat lgG anti-Rabbit lgG (H+L)-HRPO & Dianova, Hamburg & $111-035-003$ & WB 1:10,000 \\
\hline $\begin{array}{l}\text { Peroxidase AffiniPure Goat Anti-Rat } \\
\lg G, F\left(a b^{\prime}\right)_{2} \text { fragment specific }\end{array}$ & $\begin{array}{l}\text { Jackson ImmunoResearch, } \\
\text { West Grove (USA) }\end{array}$ & $112-035-006$ & WB 1:10,000 \\
\hline
\end{tabular}

\subsubsection{Synthetic oligonucleotides}

All synthetic DNA oligonucleotides were purchased from Eurofins MWG Operon (Ebersberg). Ampuwa dissolved primer stocks with a final concentration of $100 \mathrm{pmol} / \mu \mathrm{l}$ were stored at $-20^{\circ} \mathrm{C}$. Primers were used at a concentration of $10 \mathrm{pmol} / \mu \mathrm{l}$ for $\mathrm{PCR}$ experiments.

\subsubsection{Synthetic oligonucleotides for genotyping PCR experiments}

\begin{tabular}{l|l|l|c|c}
\hline Name & Direction & Primer sequence 5' $\rightarrow \mathbf{3}^{\prime}$ & $\begin{array}{c}\text { Annealing } \\
\left({ }^{\circ} \mathbf{C}\right)\end{array}$ & Cycles \\
\hline \multirow{2}{*}{ Whi-Genotype } & forward & CCTGCTGATGACTGGAAGAAAT & 65 & 35 \\
\cline { 2 - 3 } & reverse & CAAAGAAAAGTTCCCAGCAAAC & & \\
\hline
\end{tabular}

\subsubsection{Synthetic oligonucleotides for In-Fusion ${ }^{\circledR}$ experiments}

\begin{tabular}{l|l|l|c|c}
\hline Name & Direction & Primer sequence 5' $\rightarrow \mathbf{3}^{\prime}$ & $\begin{array}{c}\text { Annealing } \\
\left({ }^{\circ} \mathbf{C}\right)\end{array}$ & Cycles \\
\hline hSEMA3A-CMV-S-1 & forward & $\begin{array}{l}\text { ACGCTCTTATGGCCATGGAGGCCATG } \\
\text { GGCTGGTTAACTAGGATTG }\end{array}$ & 60 & 35 \\
\hline hSEMA3A-CMV-E-1 & reverse & $\begin{array}{l}\text { TCGGTCGACCGAATTCCTAGAGGTAAT } \\
\text { GCAGCTCAGACACT }\end{array}$ & \\
\hline
\end{tabular}




\begin{tabular}{l|l|l|l|l}
\hline hSEMA3A-GAD-S-1 & forward & $\begin{array}{l}\text { ACGCTCATATGGCCATGGAGGCCATG } \\
\text { GGCTGGTTAACTAGGATTG }\end{array}$ & 60 & 35 \\
\hline hSEMA3A-GAD-E-1 & reverse & $\begin{array}{l}\text { CACCCGGGTGGAATTCCTAGAGGTAA } \\
\text { TGCAGCTCAGACACT }\end{array}$ & 6 \\
\hline
\end{tabular}

\subsubsection{Synthetic oligonucleotides for mutagenesis experiments}

\begin{tabular}{l|l|l|c|c}
\hline Name & Direction & Primer sequence 5' $\rightarrow 3^{\prime}$ & $\begin{array}{c}\text { Annealing } \\
\left({ }^{\circ} \mathbf{C}\right)\end{array}$ & Cycles \\
\hline \multirow{2}{*}{ SEMA3A_c196t } & forward & $\begin{array}{l}\text { CTTCCTTTTGGATGAGGAATGGAGTAG } \\
\text { GCTGTATGTT }\end{array}$ & 60 & 20 \\
\cline { 2 - 5 } SEMA3A_a2002g & reverse & $\begin{array}{l}\text { AACATACAGCCTACTCCATTCCTCATC } \\
\text { CAAAAGGAAG }\end{array}$ & $\begin{array}{l}\text { AGGTAACCCTGGAAGTCGTTGACACA } \\
\text { GAGCATTTG }\end{array}$ & 60 \\
\cline { 2 - 4 } & forward & $\begin{array}{l}\text { CAAATGCTCTGTGTCAACGACTTCCAG } \\
\text { GGTTACCT }\end{array}$ & 20 \\
\hline
\end{tabular}

\subsubsection{Synthetic oligonucleotides for mycoplasma contamination}

\begin{tabular}{l|l|l|c|c}
\hline Name & Direction & Primer sequence 5' $\rightarrow \mathbf{3}^{\prime}$ & $\begin{array}{c}\text { Annealing } \\
\left({ }^{\circ} \mathbf{C}\right)\end{array}$ & Cycles \\
\hline \multirow{2}{*}{ Myco1 } & forward & ACACCATGGGAGCTGGTAAT & 55 & 35 \\
\cline { 2 - 3 } & reverse & CTTCWTCGACTTYCAGACCCAAGGCAT & 55 \\
\hline
\end{tabular}

\subsubsection{Synthetic oligonucleotides for patient screening}

\begin{tabular}{l|l|l|c|c}
\hline Name & Direction & Primer sequence 5' $\rightarrow \mathbf{3}^{\prime}$ & $\begin{array}{c}\text { Annealing } \\
\left({ }^{\circ} \mathbf{C}\right)\end{array}$ & Cycles \\
\hline \multirow{2}{*}{ SEMA3A 1 } & forward & TCACCTGTTACCTCCAGTTTCC & $65-1$ & 5 \\
\cline { 2 - 3 } & reverse & GGTTTGATGATTTGGGGTTG & 60 & 35 \\
\hline \multirow{2}{*}{ SEMA3A 2 } & forward & TCAGGTTACCATGTACCAACAGTC & $65-1$ & 5 \\
\cline { 2 - 3 } & reverse & TGCATCCATTGATTCATTACTGTC & 60 & 35 \\
\hline \multirow{2}{*}{ SEMA3A 3 } & forward & ACTTCAGTTGCCCCAATGT & $65-1$ & 5 \\
\cline { 2 - 3 } & reverse & CACATGACCCACAAGTGGAA & 60 & 35 \\
\hline \multirow{2}{*}{ SEMA3A 4 } & forward & CAGTCATGCTGATTGCTGAAA & $65-1$ & 5 \\
\cline { 2 - 3 } & reverse & TGAACCACAAGCAAAATAAACTG & 60 & 35 \\
\hline
\end{tabular}




\begin{tabular}{|c|c|c|c|c|}
\hline \multirow{2}{*}{ SEMA3A 5} & forward & TCTCTGATTAACTGATGTGTTGAAA & $65-1$ & 5 \\
\hline & reverse & TTATTTCATAATGGAAAATCTTGGT & 60 & 35 \\
\hline \multirow{2}{*}{ SEMA3A 6} & forward & CATGGTCATAACATGAAACTTGC & $65-1$ & 5 \\
\hline & reverse & CCATCATGAAGTCACCACCA & 60 & 35 \\
\hline \multirow{2}{*}{ SEMA3A 7} & forward & TGGACTGTTCAGAATGGTATTATTT & $65-1$ & 5 \\
\hline & reverse & CCTGTACCTGTATATTTGACTGCG & 60 & 35 \\
\hline \multirow{2}{*}{ SEMA3A 8/9 } & forward & GGAACGATTCGACCACAAAT & $65-1$ & 5 \\
\hline & reverse & TGCAAATTATGAGTACTTGGATAGC & 60 & 35 \\
\hline \multirow{2}{*}{ SEMA3A 10} & forward & GCCATTTTCACCTATGCCTT & $65-1$ & 5 \\
\hline & reverse & TCTGTCTGTAGCTGCATTGTTTT & 60 & 35 \\
\hline \multirow{2}{*}{ SEMA3A 11} & forward & GAACCATTGAGGCCATGTGT & $65-1$ & 5 \\
\hline & reverse & CCAACCCCTGAGATGTTCAA & 60 & 35 \\
\hline \multirow{2}{*}{ SEMA3A 12} & forward & AAAAGGAAGACCGATATCAAAGG & $65-1$ & 5 \\
\hline & \begin{tabular}{|l|} 
reverse \\
\end{tabular} & TGAGAAAACAAAATATGAGCCAAA & 60 & 35 \\
\hline \multirow{2}{*}{ SEMA3A 13} & forward & AGCAATAACCCCAACTTGGTC & $65-1$ & 5 \\
\hline & reverse & ATCAAAAACATGAGGGCAATG & 60 & 35 \\
\hline \multirow{2}{*}{ SEMA3A 14} & forward & AGAAGGCCTTTAAAGAAATTAGCA & $65-1$ & 5 \\
\hline & reverse & TTGATGCACTTATTTGAAGAAAGC & 60 & 35 \\
\hline \multirow{2}{*}{ SEMA3A 15} & forward & TCTGGTAGTGAAAAAGCCATGA & $65-1$ & 5 \\
\hline & reverse & TCTCTTCGGCTGCATTTCTT & 60 & 35 \\
\hline \multirow{2}{*}{ SEMA3A 16} & forward & TGGCAATAACTTGTCTCCTGAA & $65-1$ & 5 \\
\hline & reverse & TGAATGAGCGATTGATTGGT & 60 & 35 \\
\hline \multirow{3}{*}{ SEMA3A 17} & forward & ACAGACACGGAGTTTCAGAGC & \multirow{3}{*}{$\begin{array}{c}65-1 \\
60\end{array}$} & \multirow{3}{*}{$\begin{array}{c}5 \\
35\end{array}$} \\
\hline & reverse & AGATTGGGGTGGTTGATGAG & & \\
\hline & reverse & CCCACCATTGTAAACATCCA & & \\
\hline
\end{tabular}

\subsubsection{Synthetic oligonucleotides for RT-PCR experiments}

\begin{tabular}{l|l|l|c|c}
\hline Name & Direction & Primer sequence 5' $\rightarrow 3^{\prime}$ & $\begin{array}{c}\text { Annealing } \\
\left({ }^{\circ} \mathbf{C}\right)\end{array}$ & Cycles \\
\hline \multirow{2}{*}{ Actg2 } & forward & GGCTTTGCAGGAGATGATGC & $64-1$ & 5 \\
\cline { 2 - 3 } & reverse & GGCAGTGCATAGCCCTCATA & 59 & 25 \\
\hline \multirow{2}{*}{ mChd7_qRT-5 } & forward & GCAGTCAGAGCAGCAGGAAT & $65-1$ & 5 \\
\cline { 2 - 3 } & reverse & GCTCTGGCCCTGTTTTGACT & 60 & 30 \\
\hline \multirow{2}{*}{ Cnn1 } & forward & TGGCCCAGAAATACGACCAT & $64-1$ & 5 \\
\cline { 2 - 3 } Col2a1 & reverse & CCTTTTGTCTTGGCCATGCT & 59 & 25 \\
\cline { 2 - 3 } & forward & GAAGAGCGGAGACTACTGGATT & $64-1$ & 5 \\
\cline { 2 - 3 } & reverse & AGGTAGGCGATGCTGTTCTT & 59 & 25 \\
\hline
\end{tabular}




\begin{tabular}{|c|c|c|c|c|}
\hline \multirow{2}{*}{ Gfap } & forward & GCCTCGGTCCTAGTCGAC & $64-1$ & 5 \\
\hline & reverse & TCCTGTCTATACGCAGCCAG & 59 & 25 \\
\hline \multirow{2}{*}{ Nefh } & forward & GCAGCCAAAGTGAACACAGA & $64-1$ & 5 \\
\hline & reverse & TGTAAGCGGCAATCTCAATGT & 59 & 25 \\
\hline \multirow{2}{*}{ mNrp1RT } & forward & CCACAGAGAAGCCAACCATT & $65-1$ & 5 \\
\hline & reverse & TGAAGTTGCCATCTCCTGTATG & 60 & 30 \\
\hline \multirow{2}{*}{ mNrp2RT } & forward & GGGGAAAACTGCAGCTTTGA & $65-1$ & 5 \\
\hline & reverse & GGCCATCACTCTGCAGTTTC & 60 & 30 \\
\hline \multirow{2}{*}{ P75 } & forward & CTCAGATGAAGCCAACCACG & $63-1$ & 5 \\
\hline & reverse & GGAGCAATAGACAGGAATGAGG & 58 & 30 \\
\hline \multirow{2}{*}{ Pax3_Seaberg } & forward & GGAGGCGGATCTAGAAAGGAAGGA & $64-1$ & 5 \\
\hline & reverse & CCCCCGGAATGAGATGGTTGAA & 59 & 25 \\
\hline \multirow{2}{*}{ PlxnA1RT } & forward & TTGGAATGTGCCTGACCTCT & $65-1$ & 5 \\
\hline & reverse & TGAGGTAGAGCTTCACCACC & 60 & 30 \\
\hline \multirow{2}{*}{ mSema3a_RT_3 } & forward & TGCAGTCCGAAGTCACAGAG & $65-1$ & 5 \\
\hline & reverse & TTGCATGAATCCGTGTTCCAC & 60 & 30 \\
\hline \multirow{2}{*}{ mSema3c_RT_3 } & forward & GGATCGGCAGTGTGTGTGTA & $65-1$ & 5 \\
\hline & reverse & ACAACGTCATCTGGGAAGTCC & 60 & 30 \\
\hline \multirow{2}{*}{ mSema3d_RT_2 } & forward & AAGGCTCTGCTGTCTGTGTG & $68-1$ & 5 \\
\hline & reverse & CTGGAAAGTCTCGGGTGGAC & 63 & 30 \\
\hline \multirow{2}{*}{ mSema3e_RT_4 } & forward & GCATGGGGAAGTTAGGCCAT & $68-1$ & 5 \\
\hline & reverse & CTCGGGTGTAGATCGTGTGG & 63 & 30 \\
\hline \multirow{2}{*}{ Slug } & forward & TGCCATCGAAGCTGAGAAGT & $64-1$ & 5 \\
\hline & reverse & GCAGAAGCGACATTCTGGAG & 59 & 25 \\
\hline \multirow{2}{*}{ Snail_Seaberg } & forward & AAGCCCAACTATAGCGAGCTG & $64-1$ & 5 \\
\hline & reverse & AGTTGAAGATCTTCCGCGACT & 59 & 25 \\
\hline \multirow{2}{*}{ Sox10 } & forward & CCCTTCATTGAGGAGGCTGA & $63-1$ & 5 \\
\hline & reverse & TTGGTCCAGCTCAGTCACAT & 58 & 30 \\
\hline \multirow{2}{*}{ Twist_26.01.15 } & forward & GCCAGGTACATCGACTTCCTG & $64-1$ & 5 \\
\hline & reverse & CCACGCCCTGATTCTTGTGA & 59 & 25 \\
\hline \multirow{2}{*}{ Tyr } & forward & AGCATGCACAATGCCTTACA & $65-1$ & 5 \\
\hline & reverse & GAGCGGTATGAAAGGAACCA & 60 & 30 \\
\hline
\end{tabular}




\subsubsection{Synthetic oligonucleotides for sequencing}

\begin{tabular}{|c|c|c|c|c|}
\hline Name & Direction & Primer sequence $5^{\prime} \rightarrow 3^{\prime}$ & $\begin{array}{c}\text { Annealing } \\
\left({ }^{\circ} \mathrm{C}\right)\end{array}$ & Cycles \\
\hline \multirow{2}{*}{ seq_hSEMA3A-1 } & forward & ATGGGCTGGTTAACTAGGATTG & \multirow{2}{*}{60} & \multirow{2}{*}{30} \\
\hline & reverse & CACATTGTTCTTCCCATTCTGA & & \\
\hline \multirow{2}{*}{ seq_hSEMA3A-2 } & forward & AATGGACAACATTCCTCA & \multirow{2}{*}{60} & \multirow{2}{*}{30} \\
\hline & reverse & GGAATACATCCTGCAGTTCATC & & \\
\hline \multirow{2}{*}{ seq_hSEMA3A-3n } & forward & AGACGCACAAGACGACAAGATA & \multirow{2}{*}{60} & \multirow{2}{*}{30} \\
\hline & reverse & ATTCTCTCTTCAGGGCTGTGG & & \\
\hline seq_hSEMA3A-4 & forward & GGGAACAGTAACAAATGGAAGC & 60 & 30 \\
\hline \multirow{2}{*}{ seq_hSEMA3A-5 } & forward & GACAGAGCAGCATGATTCCA & \multirow{2}{*}{60} & \multirow{2}{*}{30} \\
\hline & reverse & ATGGCTGGATGACTTCTTGC & & \\
\hline \multirow{2}{*}{ seq_hSEMA3A-6 } & forward & AGAGGTTCTGCTGGAAGAAATG & \multirow{2}{*}{60} & \multirow{2}{*}{30} \\
\hline & reverse & ATTGCCTGAATCCTTCTGTTGT & & \\
\hline \multirow{2}{*}{ seq_mSema3A-1 } & forward & CACTGGGATTGCCTGTCTTT & \multirow{2}{*}{60} & \multirow{2}{*}{30} \\
\hline & reverse & GGCCAAGCCATTAAAAGTGA & & \\
\hline \multirow{2}{*}{ seq_mSema3A-2 } & forward & CGGGACTTCGCTATCTTCAG & \multirow{2}{*}{60} & \multirow{2}{*}{30} \\
\hline & reverse & GATGAGATGGGCACTGATGA & & \\
\hline \multirow{2}{*}{ seq_mSema3A-3 } & forward & ACATTTGGCGGATTTGACTC & \multirow{2}{*}{60} & \multirow{2}{*}{30} \\
\hline & reverse & CTGCATCCACTCGGTCTACA & & \\
\hline \multirow{2}{*}{ seq_mSema3A-4 } & forward & GCGCACAAGACGACAAGATA & \multirow{2}{*}{60} & \multirow{2}{*}{30} \\
\hline & reverse & TCTGTGACTTCGGACTGCAT & & \\
\hline \multirow{2}{*}{ seq_mSema3A-5 } & forward & GTGGAAGCACATGCAAGAGA & \multirow{2}{*}{60} & \multirow{2}{*}{30} \\
\hline & reverse & AACTCGTGGGTCCTCCTGT & & \\
\hline \multirow{2}{*}{$\mathrm{pCMV}$} & forward & CTCAGTGGATGTTGCCTTTACTTCT & \multirow{2}{*}{60} & \multirow{2}{*}{30} \\
\hline & reverse & AAGCAATAGCATCACAAATTTCACA & & \\
\hline T7cDNA3 & forward & TAATACGACTCACTATAGG & 60 & 30 \\
\hline
\end{tabular}

\subsubsection{Synthetic oligonucleotides for qRT-PCR experiments}

\begin{tabular}{|c|c|c|c|c|}
\hline Name & Direction & Primer sequence $5^{\prime} \rightarrow 3^{\prime}$ & $\begin{array}{c}\text { Annealing } \\
\left({ }^{\circ} \mathrm{C}\right)\end{array}$ & Cycles \\
\hline \multirow{2}{*}{ mChd7_qRT-3 } & forward & AGAAGAGAAGCTGGAGGACGA & \multirow{2}{*}{60} & \multirow{2}{*}{40} \\
\hline & reverse & TGATTTCCAGGGCCTTGTCC & & \\
\hline \multirow{2}{*}{ qRT_mHprt } & forward & AGCCCCAAAATGGTTAAGGTTGC & \multirow{2}{*}{60} & \multirow{2}{*}{40} \\
\hline & reverse & TTGCAGATTCAACTTGCGCTCAT & & \\
\hline \multirow{2}{*}{ mSema3a_RT-1 } & forward & CGGGACTTCGCTATCTTCAGAACACT & \multirow{2}{*}{60} & \multirow{2}{*}{40} \\
\hline & reverse & TCATCTTCAGGGTTGTCACTCTCTGG & & \\
\hline
\end{tabular}




\begin{tabular}{l|l|l|c|c}
\hline qRT_mTbp & forward & CCCACAACTCTTCCATTCTCAAAC & \multirow{2}{*}{60} & 4 \\
\cline { 2 - 3 } & reverse & TCAAGTTTACAGCCAAGATTCACG & & \\
\hline
\end{tabular}

\subsubsection{Molecular weight standards}

\begin{tabular}{|c|c|}
\hline Application & Standard and Manufacturer \\
\hline DNA molecular weight standards & $\begin{array}{l}100 \text { bp DNA Ladder (Invitrogen, Karlsruhe) } \\
1 \text { kb DNA Ladder (Invitrogen, Karlsruhe) } \\
1 \mathrm{~kb} \text { Plus DNA Ladder (Invitrogen, Karlsruhe) } \\
\text { DNA molecular weight standards were diluted } \\
\text { with loading buffer according to manufacturer's } \\
\text { instructions. }\end{array}$ \\
\hline Protein molecular weight standards & $\begin{array}{l}\text { SeeBlue }{ }^{\circledR} \text { Plus2 Pre-Stained Protein Standard } \\
\text { (Invitrogen, Karlsruhe) } \\
\text { HiMark }^{\mathrm{TM}} \text { Pre-Stained High Molecular Weight } \\
\text { Protein Standard (Invitrogen, Karlsruhe) }\end{array}$ \\
\hline
\end{tabular}

\subsubsection{Animals/ethic statement}

The used mouse line Whirligig (Bosman et al., 2005) was kindly provided from K. P. Steel (Sanger Centre, Cambridge, United Kingdom) and the Helmholtz Zentrum (Munich). The mice were housed in the animal facility of the Institute of Human Genetics (Göttingen) under conventional conditions $\left(12 \mathrm{~h}\right.$ light/dark cycle, $22^{\circ} \mathrm{C}, 55 \pm 5 \%$ relative humidity and free access to food and water). Animal food was purchased from ssniff Spezialdiäten (Soest). All experiments were approved by the Institutional Animal Care and Use Committee of the University of Göttingen and conducted according to the European and German protection of animal act. The number of sacrificed animals and the stress and pain the mice were suffering was kept to a minimum. Euthanasia of mice was performed by $\mathrm{CO}_{2}$-asphyxation or cervical dislocation. 


\subsubsection{Patient samples}

Genomic DNA of patient samples was provided by the genetic diagnostics laboratory of the Institute of Human Genetics (University Medical Center Göttingen) and by collaboration with Conny van Ravenswaaij-Arts of the Department of Genetics (University Medical Center Groningen, The Netherlands). The ethical approval was obtained from the Ethics Committee of the University Göttingen.

\subsubsection{Databases / Online tools / Programs}

\begin{tabular}{|c|c|}
\hline Analysis of / Designing of / Usage for & Database / Online tool / Program \\
\hline Gene domains & $\begin{array}{l}\text { SMART } \\
\text { http://smart.embl-heidelberg.de } \\
\text { UniProtKB } \\
\text { http://www.uniprot.org/ }\end{array}$ \\
\hline Genome variants & $\begin{array}{l}\text { Exome Aggregation Consortium (ExAC) Browser } \\
\text { http://exac.broadinstitute.org/ }\end{array}$ \\
\hline $\begin{array}{l}\text { Information about human genes and genetic } \\
\text { disorders }\end{array}$ & $\begin{array}{l}\text { Online Mendelian Inheritance in Man (OMIM) } \\
\text { https://www.omim.org/ }\end{array}$ \\
\hline In-Fusion ${ }^{\circledR}$ PCR Cloning System & http://bioinfo.clontech.com/infusion/ \\
\hline Mice administration & Tierbase Client 4D v15.1 \\
\hline Nucleotide exchanges & $\begin{array}{l}\text { MutationTaster } \\
\text { http://www.mutationtaster.org/ } \\
\text { PolyPhen-2 } \\
\text { http://genetics.bwh.harvard.edu/pph2/ } \\
\text { SIFT } \\
\text { http://sift.jcvi.org/ }\end{array}$ \\
\hline Nucleotide sequences & $\begin{array}{l}\text { Ensembl genome browser } 87 \\
\text { http://www.ensembl.org/ } \\
\text { National Center for Biotechnology Information } \\
\text { (NCBI) } \\
\text { https://www.ncbi.nlm.nih.gov/ }\end{array}$ \\
\hline
\end{tabular}




\begin{tabular}{|c|c|}
\hline Plasmid maps & $\begin{array}{l}\text { Savvy Version } 0.1 \\
\text { http://www.bioinformatics.org/savvy/ }\end{array}$ \\
\hline Primers & $\begin{array}{l}\text { Primer3 } \\
\text { http://bioinfo.ut.ee/primer3-0.4.0/ }\end{array}$ \\
\hline Protein molecular weights & $\begin{array}{l}\text { ExPASy } \\
\text { http://web.expasy.org/compute_pi/ }\end{array}$ \\
\hline Repetitive gene sequences & $\begin{array}{l}\text { RepeatMasker } \\
\text { http://www.repeatmasker.org/ }\end{array}$ \\
\hline Restriction sites & $\begin{array}{l}\text { NEBcutter V2.0 } \\
\text { http://nc2.neb.com/NEBcutter2/ }\end{array}$ \\
\hline Searching for publications & $\begin{array}{l}\text { PubMed } \\
\text { https://www.ncbi.nlm.nih.gov/pubmed/ }\end{array}$ \\
\hline Sequences & $\begin{array}{l}\text { Chromas Lite } 2.01 \text { (Technelysium Pty Ltd.) } \\
\text { Sequence Pilot 4.3.1 (JSI medical systems) }\end{array}$ \\
\hline Sequence alignments & $\begin{array}{l}\text { Basic Local Alignment Search Tool (BLAST) } \\
\text { provided by NCBI } \\
\text { https://blast.ncbi.nlm.nih.gov/Blast.cgi }\end{array}$ \\
\hline
\end{tabular}

\subsubsection{Statistical analysis}

An unpaired student's $t$ test was performed to test for statistical significance. The calculated $p$-value indicates the probability that the results are not different from each other. A $p$-value smaller than * $p \leq 0.05$ means statistically significant, ${ }^{* *} p \leq 0.01$ means very significant, ${ }^{* * *} p \leq 0.001$ means extremely significant. 


\section{$2.2 \quad$ Methods}

\subsubsection{Molecular biology methods}

\subsubsection{Isolation of nucleic acids}

\subsection{Isolation of genomic DNA for mouse genotyping}

For mouse genotyping, genomic DNA (gDNA) was isolated from tail biopsy taken at the age of four weeks. The tail biopsy was mixed with $200 \mu \mathrm{l}$ of DirectPCR ${ }^{\circledR}$ Lysis Reagent Tail (Peqlab) and $8 \mu \mathrm{l}$ of proteinase $\mathrm{K}(10 \mathrm{mg} / \mathrm{ml})$ and incubated at $55^{\circ} \mathrm{C}$ by shaking at $950 \mathrm{rpm}$ overnight. The tail biopsy from a mouse embryo was mixed with $100 \mu \mathrm{l}$ of DirectPCR Lysis Reagent Tail and $4 \mu \mathrm{l}$ of proteinase $\mathrm{K}(10 \mathrm{mg} / \mathrm{ml})$ and incubated as previously described. The following day, proteinase $K$ was inactivated by incubation for $45 \mathrm{~min}$ at $85^{\circ} \mathrm{C}$. The samples were short centrifuged, transferred on ice and used for genotyping PCR (chapter 2.2.1.2.1.3). Isolated gDNA was finally stored at $4^{\circ} \mathrm{C}$.

\subsection{Minipreparation of plasmid DNA}

Small amounts of plasmid DNA from bacteria were isolated with the ready-to-use buffers (P1/P2/P3) for minipreparation (Qiagen). $3 \mathrm{ml}$ of LB medium containing the appropriate antibiotic was inoculated with a single bacterial colony or a small amount of a glycerol stock and incubated at $37^{\circ} \mathrm{C}$ by shaking at $160 \mathrm{rpm}$ overnight. For preparation of a glycerol stock the following day, $500 \mu \mathrm{l}$ of the bacterial culture was mixed with $320 \mu \mathrm{l}$ of $50 \%$ sterile glycerol and stored at $-80^{\circ} \mathrm{C}$. The extant overnight culture was centrifuged at $4000 \times g$ for $10 \mathrm{~min}$ at $4^{\circ} \mathrm{C}$. The bacterial cell pellet was resuspended in $200 \mu \mathrm{l}$ of $\mathrm{P} 1$ buffer, transferred into a $1.5 \mathrm{ml}$ reaction tube and $200 \mu \mathrm{l}$ of P2 buffer (modified alkaline lysis) was added. The solution was mixed by inverting and incubated for 5 min at RT. After addition of $200 \mu \mathrm{l}$ P3 buffer (neutralization) and repeated mixing by inverting, the solution was centrifuged at $16,000 \times g$ for $10 \mathrm{~min}$ at RT. The received supernatant was transferred into a new $1.5 \mathrm{ml}$ reaction tube. DNA was precipitated with $800 \mu \mathrm{l}$ of $100 \%$ isopropanol (chilled at $-20^{\circ} \mathrm{C}$ ) and repeated mixed by inverting before centrifuged at $16,000 \times \mathrm{g}$ for $30 \mathrm{~min}$ at $4^{\circ} \mathrm{C}$. The DNA pellet was washed with $300 \mu \mathrm{l}$ of $70 \%$ ethanol (chilled at $-20^{\circ} \mathrm{C}$ ) and centrifuged at $16,000 \times g$ for $5 \mathrm{~min}$ at $4^{\circ} \mathrm{C}$. After discarding the supernatant, the plasmid DNA pellet was dried at $56^{\circ} \mathrm{C}$ and resolved in an appropriate volume of Ampuwa 
by gently shaking for $10 \mathrm{~min}$ at $56^{\circ} \mathrm{C}$. Afterwards, isolated plasmid DNA was stored at $-20^{\circ} \mathrm{C}$.

\subsection{Endotoxin-free midipreparation of plasmid DNA}

Large amounts of plasmid DNA from bacteria were isolated with ready-to-use reaction system HiPure Plasmid Midiprep Kit (Invitrogen, Karlsruhe). $50 \mathrm{ml}$ of LB medium, containing the appropriate antibiotic, was inoculated with either $50 \mu \mathrm{l}$ of bacterial overnight culture (taken before minipreparation and stored at $4^{\circ} \mathrm{C}$ ) or a small amount of a glycerol stock and incubated at $37^{\circ} \mathrm{C}$ by shaking at $160 \mathrm{rpm}$ overnight. The overnight culture was centrifuged at $4000 \times \mathrm{g}$ for $10 \mathrm{~min}$ at RT. The bacterial cell pellet was resuspended in $4 \mathrm{ml}$ of R3 buffer, further mixed with $4 \mathrm{ml}$ of L7 buffer (modified alkaline lysis), inverted five times and incubated for $5 \mathrm{~min}$ at RT. After addition of $4 \mathrm{ml} \mathrm{N} 3$ buffer (neutralization) the suspension was mixed by inverting until the suspension was homogenous and centrifuged at $12,000 \times \mathrm{g}$ for $10 \mathrm{~min}$ at RT. The supernatant was loaded onto a with EQ1 buffer equilibrated column. The column was washed twice with $10 \mathrm{ml}$ of $\mathrm{W} 8$ buffer. The DNA was eluted with $5 \mathrm{ml}$ of $\mathrm{E} 4$ buffer and further precipitated by addition of $2.5 \mathrm{ml}$ $100 \%$ isopropanol (chilled at $-20^{\circ} \mathrm{C}$ ). The suspension was mixed by repeated inverting and centrifuged at $16,000 \times \mathrm{g}$ for $30 \mathrm{~min}$ at $4^{\circ} \mathrm{C}$. The DNA pellet was washed with $2.5 \mathrm{ml}$ of $70 \%$ ethanol (chilled at $-20^{\circ} \mathrm{C}$ ). After centrifugation at $16,000 \times g$ for $5 \mathrm{~min}$ at $4^{\circ} \mathrm{C}$, the supernatant was discarded and the DNA pellet was dried at $56^{\circ} \mathrm{C}$. The plasmid DNA pellet was resolved in an appropriate volume of Ampuwa by shaking for $10 \mathrm{~min}$ at $56^{\circ} \mathrm{C}$ and finally stored at $-20^{\circ} \mathrm{C}$.

\subsection{Isolation of total RNA from cultured cells}

For total RNA isolation from cultured cells either fresh or at $-80^{\circ} \mathrm{C}$ stored cell pellets were used. Pellet was thoroughly mixed with $1 \mathrm{ml}$ of Trizol Reagent (Invitrogen), incubated for 5 min at RT and $200 \mu \mathrm{l}$ of chloroform was added. The suspension was vigorously mixed for $15 \mathrm{~s}$, incubated for $15 \mathrm{~min}$ at RT and centrifuged at $18,600 \times \mathrm{g}$ for $15 \mathrm{~min}$ at $4^{\circ} \mathrm{C}$. After centrifugation, the upper aqueous phase containing the RNA was transferred into a new reaction tube. For precipitation of the RNA $500 \mu \mathrm{l}$ of $100 \%$ isopropanol (chilled at $-20^{\circ} \mathrm{C}$ ) was added, the suspension was mixed by inverting, incubated for 10 min before 
centrifuged at $18,600 \times \mathrm{g}$ for $8 \mathrm{~min}$ at $4^{\circ} \mathrm{C}$. The RNA pellet was washed with $1 \mathrm{ml}$ of $75 \%$ ethanol/DEPC- $\mathrm{H}_{2} \mathrm{O}$ and centrifuged at $18,600 \times \mathrm{g}$ for $5 \mathrm{~min}$ at $4^{\circ} \mathrm{C}$. The supernatant was discarded and the RNA pellet was dried at $60^{\circ} \mathrm{C}$. For resolving the pellet, an appropriate volume of DEPC- $\mathrm{H}_{2} \mathrm{O}$ was used by shaking for $10 \mathrm{~min}$ at $60^{\circ} \mathrm{C}$. The isolated RNA was stored at $-80^{\circ} \mathrm{C}$.

\subsection{Isolation of RNA from mouse tissues}

The mouse tissue was initially homogenized using the TissueLyser LT (Qiagen). Together with a stainless steel bead (ø $5 \mathrm{~mm}$, Qiagen) and $1 \mathrm{ml}$ of Trizol Reagent (Invitrogen) a small sample of the tissue was transferred into a $2 \mathrm{ml}$ reaction tube. The tissue was homogenized in the TissueLyser LT for $5 \mathrm{~min}$ at 50 1/s oscillation. Afterwards, the reaction tube was kept on ice for $5 \mathrm{~min}$. After centrifugation at $16,000 \times \mathrm{g}$ for $10 \mathrm{~min}$ at $4^{\circ} \mathrm{C}$, the lysed cells containing supernatant was transferred into a new $1.5 \mathrm{ml}$ reaction tube. The RNA isolation was continued by using the total RNA isolation protocol from cultured cells (chapter 2.2.1.1.4) starting with addition of $200 \mu \mathrm{l}$ chloroform to the supernatant with the lysed cells.

\subsection{Determination of nucleic acid concentration}

Nucleic acid concentrations and purities were determined by measuring the absorption at $260 \mathrm{~nm}$ using the NanoDrop 2000c UV-Vis spectrophotometer (Peqlab) according to manufacturer's instructions. Contaminations of protein and salt were estimated with the absorbance ratios of $A_{260 / 280}$ and $A_{260 / 230}$, respectively.

\subsubsection{Polymerase Chain Reactions}

\subsection{Amplification of DNA}

The polymerase chain reaction (PCR) represents a standard in vitro method to amplify specific DNA fragments (Mullis et al., 1986). The double stranded template DNA is denatured at high temperatures (denaturation). This allows short oligonucleotides (primers) to bind to the now single stranded DNA (annealing). Bound oligonucleotides are 
extended by a polymerase (elongation). Periodic repetition of denaturation, annealing and elongation results in an exponential increase of specific DNA fragments. The result of the PCR can be visualized by agarose gel electrophoresis (chapter 2.2.1.3).

\subsection{Standard PCR}

The standard PCR was used to control the efficiency of cDNA synthesis from RNA (chapter 2.2.1.2.2). Specific primers for the housekeeping gene mouse Hprt (hypoxanthine-guanine phosphoribosyltransferase) (chapter 2.1.12.8) and the Q5 ${ }^{\circledR} \mathrm{High}$ Fidelity DNA Polymerase (New England Biolabs) with the supplied reagents were used.

The following reagents for $25 \mu$ of reaction mixture were pipetted into a PCR reaction tube:

\begin{tabular}{l|r}
\hline \multicolumn{1}{c|}{ Component } & \multicolumn{1}{c}{ Volume } \\
\hline cDNA & $1.0 \mu \mathrm{l}$ \\
\hline Q5 Reaction Buffer (5x) & $5.0 \mu \mathrm{l}$ \\
\hline Q5 High GC Enhancer (5x) & $5.0 \mu \mathrm{l}$ \\
\hline Primer forward (10 pmol/ $\mu \mathrm{l})$ & $1.25 \mu \mathrm{l}$ \\
\hline Primer reverse $(10 \mathrm{pmol} / \mu \mathrm{l})$ & $1.25 \mu \mathrm{l}$ \\
\hline dNTP mix (10 mM) & $0.5 \mu \mathrm{l}$ \\
\hline Q5 High-Fidelity DNA Polymerase $(2 \mathrm{U} / \mu \mathrm{l})$ & $0.25 \mu \mathrm{l}$ \\
\hline Ampuwa & $10.75 \mu \mathrm{l}$ \\
\hline
\end{tabular}

The reaction mixture was incubated in a thermocycler using the following standard PCR program:

\begin{tabular}{l|c|c|c}
\hline \multicolumn{1}{c|}{ Step } & Temperature & Time & Repeats \\
\hline Initial denaturation & $98^{\circ} \mathrm{C}$ & $30 \mathrm{~s}$ & 1 \\
\cline { 1 - 3 } Denaturation & $98^{\circ} \mathrm{C}$ & $10 \mathrm{~s}$ & \multirow{2}{*}{25} \\
\cline { 1 - 2 } Annealing & $60^{\circ} \mathrm{C}$ & $30 \mathrm{~s}$ & \\
\cline { 1 - 3 } Elongation & $72^{\circ} \mathrm{C}$ & $20 \mathrm{~s}$ & 1 \\
\cline { 1 - 3 } Final elongation & $72^{\circ} \mathrm{C}$ & 2 min & 1 \\
\hline Storage & $4^{\circ} \mathrm{C}$ & $\infty$ & \\
\hline
\end{tabular}


After completion of the thermocycler program, loading buffer was added to $5 \mu \mathrm{l}$ of the reaction mixture and loaded on a 1.5\% agarose gel together with 100 bp DNA Ladder (Invitrogen).

\subsection{Touchdown PCR}

Generally, the components of the reaction mixture were the same as for standard PCR (chapter 2.2.1.2.1.1) with the exception of the touchdown PCR using patients DNA. To increase specificity, sensitivity and yield of PCR, a touchdown PCR program was applied.

The reaction mixture was incubated in a thermocycler using the following touchdown PCR program:

\begin{tabular}{l|c|c|c}
\hline \multicolumn{1}{c|}{ Step } & Temperature & Time & Repeats \\
\hline Initial denaturation & $98^{\circ} \mathrm{C}$ & $30 \mathrm{~s}$ & 1 \\
\hline Denaturation & $98^{\circ} \mathrm{C}$ & $10 \mathrm{~s}$ & \multirow{2}{*}{5} \\
\cline { 1 - 2 } Annealing & $(2.1 .12 .6)-1^{\circ} \mathrm{C}$ & $30 \mathrm{~s}$ & \\
\cline { 1 - 3 } Elongation & $72^{\circ} \mathrm{C}$ & $20 \mathrm{~s}$ & \multirow{2}{*}{$(2.1 .12 .6)$} \\
\cline { 1 - 3 } Denaturation & $98^{\circ} \mathrm{C}$ & $10 \mathrm{~s}$ & \\
\hline Annealing & $(2.1 .12 .6)$ & $30 \mathrm{~s}$ & 1 \\
\hline Elongation & $72^{\circ} \mathrm{C}$ & $20 \mathrm{~s}$ & 1 \\
\hline Final elongation & $72^{\circ} \mathrm{C}$ & $2 \mathrm{~min}$ & \\
\cline { 1 - 3 } Storage & $4^{\circ} \mathrm{C}$ & $\infty$ & \\
\hline
\end{tabular}

After completion of the thermocycler program, loading buffer was added to $3-5 \mu \mathrm{l}$ of the reaction mixture and loaded on a 1.5\% agarose gel together with 100 bp DNA Ladder (Invitrogen).

Touchdown PCR with patients DNA samples for mutational analysis

For touchdown PCR with patient DNA samples the HotStarTaq ${ }^{\circledR}$ Plus Master Mix Kit (Qiagen) and specific primers (chapter 2.1.12.5) were used. 
The following reagents for $25.5 \mu \mathrm{l}$ of reaction mixture were pipetted into a 96 -well plate:

\begin{tabular}{l|r}
\hline \multicolumn{1}{c|}{ Component } & \multicolumn{1}{c}{ Volume } \\
\hline gDNA & $1.0 \mu \mathrm{l}$ \\
\hline Primer forward $(10 \mathrm{pmol} / \mu \mathrm{l})$ & $1.0 \mu \mathrm{l}$ \\
\hline Primer reverse $(10 \mathrm{pmol} / \mu \mathrm{l})$ & $1.0 \mu \mathrm{l}$ \\
\hline HotStarTaq Plus Master Mix & $12.5 \mu \mathrm{l}$ \\
\hline Ampuwa & $10.0 \mu \mathrm{l}$ \\
\hline
\end{tabular}

The reaction mixture was incubated in a thermocycler using the following touchdown PCR program:

\begin{tabular}{l|c|c|c}
\hline \multicolumn{1}{c|}{ Step } & Temperature & Time & Repeats \\
\hline Initial denaturation & $95^{\circ} \mathrm{C}$ & $7 \mathrm{~min}$ & 1 \\
\hline Denaturation & $95^{\circ} \mathrm{C}$ & $30 \mathrm{~s}$ & \multirow{2}{*}{5} \\
\cline { 1 - 3 } Annealing & $(2.1 .12 .5)-1^{\circ} \mathrm{C}$ & $1 \mathrm{~min}$ & \\
\cline { 1 - 3 } Elongation & $72^{\circ} \mathrm{C}$ & $1 \mathrm{~min}$ & \multirow{2}{*}{$(2.1 .12 .5)$} \\
\cline { 1 - 3 } Denaturation & $95^{\circ} \mathrm{C}$ & $30 \mathrm{~s}$ & \\
\hline Annealing & $(2.1 .12 .5)$ & $1 \mathrm{~min}$ & 1 \\
\hline Elongation & $72^{\circ} \mathrm{C}$ & $1 \mathrm{~min}$ & 1 \\
\hline Final elongation & $72^{\circ} \mathrm{C}$ & $5 \mathrm{~min}$ & \\
\cline { 1 - 3 } Storage & $4^{\circ} \mathrm{C}$ & $\infty$ & \\
\hline
\end{tabular}

After completion of the thermocycler program, loading buffer was added to $3 \mu \mathrm{l}$ of the reaction mixture and loaded on a $1.0 \%$ agarose gel together with $1 \mathrm{~kb}$ Plus DNA Ladder (Invitrogen).

\subsection{Genotyping PCR}

The genotyping PCR was used for mouse genotyping using gDNA isolated from tail biopsy (chapter 2.2.1.1.1). Specific primers for the DNA region which contains the nonsense mutation c.2918G $>A$ ( $p . W 973 X)$ in case of the heterozygous or homozygous genotype (chapter 2.1.12.1) and the Immolase ${ }^{\mathrm{TM}}$ DNA Polymerase (Bioline) with the supplied reagents were used. 
The following reagents for $50 \mu \mathrm{l}$ of reaction mixture were pipetted into a PCR reaction tube:

\begin{tabular}{l|c}
\hline \multicolumn{1}{c|}{ Component } & Volume \\
\hline gDNA & $2.0 \mu \mathrm{l}$ \\
\hline ImmoBuffer (10x) & $5.0 \mu \mathrm{l}$ \\
\hline $\mathrm{MgCl}_{2}$ Solution $(50 \mathrm{mM})$ & $1.5 \mu \mathrm{l}$ \\
\hline Primer forward $(10 \mathrm{pmol} / \mu \mathrm{l})$ & $1.0 \mu \mathrm{l}$ \\
\hline Primer reverse $(10 \mathrm{pmol} / \mu \mathrm{l})$ & $1.0 \mu \mathrm{l}$ \\
\hline dNTP mix $(10 \mathrm{mM})$ & $1.0 \mu \mathrm{l}$ \\
\hline Immolase DNA Polymerase $(5 \mathrm{U} / \mu \mathrm{l})$ & $0.4 \mu \mathrm{l}$ \\
\hline Ampuwa & $38.1 \mu \mathrm{l}$ \\
\hline
\end{tabular}

The reaction mixture was incubated in a thermocycler using the following standard PCR program:

\begin{tabular}{l|c|c|c}
\hline \multicolumn{1}{c|}{ Step } & Temperature & Time & Repeats \\
\hline Initial denaturation & $94^{\circ} \mathrm{C}$ & $5 \mathrm{~min}$ & 1 \\
\hline Denaturation & $94^{\circ} \mathrm{C}$ & $30 \mathrm{~s}$ & \multirow{2}{*}{35} \\
\hline Annealing & $65^{\circ} \mathrm{C}$ & $45 \mathrm{~s}$ & \\
\hline Elongation & $72^{\circ} \mathrm{C}$ & $1 \mathrm{~min}$ & \\
\hline Final elongation & $72^{\circ} \mathrm{C}$ & $7 \mathrm{~min}$ & 1 \\
\hline Storage & $4^{\circ} \mathrm{C}$ & $\infty$ & 1 \\
\hline
\end{tabular}

After completion of the thermocycler program, loading buffer was added to $3 \mu \mathrm{l}$ of the reaction mixture and loaded on a 1.5\% agarose gel together with 100 bp DNA Ladder (Invitrogen). The purified PCR product was further used for sequencing analysis (chapter 2.2.1.2.4) to distinguish between the wildtype, heterozygous and homozygous state of Whirligig mice.

\subsection{Mutagenesis PCR}

The mutagenesis PCR was used to create specific, targeted changes in double stranded plasmid DNA with the QuikChange II XL Site-Directed Mutagenesis Kit according to 
manufacturer's instructions (chapter 2.2.1.4.4). Specific primers for mutagenesis (chapter 2.1.12.3) and the PfuUltra ${ }^{\circledR}$ High-Fidelity DNA Polymerase (Agilent Technologies) with the supplied reagents were used.

The following reagents for $50 \mu \mathrm{l}$ of reaction mixture were pipetted into a PCR reaction tube:

\begin{tabular}{l|r}
\hline \multicolumn{1}{c|}{ Component } & \multicolumn{1}{c}{ Volume } \\
\hline Double stranded plasmid DNA & $(75 \mathrm{ng}) \times \mu \mathrm{l}$ \\
\hline Reaction buffer $(10 \mathrm{x})$ & $5.0 \mu \mathrm{l}$ \\
\hline QuikSolution & $3.0 \mu \mathrm{l}$ \\
\hline Primer forward $(125 \mathrm{ng} / \mu \mathrm{l})$ & $1.0 \mu \mathrm{l}$ \\
\hline Primer reverse $(125 \mathrm{ng} / \mu \mathrm{l})$ & $1.0 \mu \mathrm{l}$ \\
\hline dNTP mix & $2.0 \mu \mathrm{l}$ \\
\hline PfuUltra High-Fidelity DNA Polymerase $(2.5 \mathrm{U} / \mu \mathrm{l})$ & $1.0 \mu \mathrm{l}$ \\
\hline Ampuwa & $\mathrm{x \mu l}$ \\
\hline
\end{tabular}

The reaction mixture was incubated in a thermocycler using the following PCR program:

\begin{tabular}{l|c|c|c}
\hline \multicolumn{1}{c|}{ Step } & Temperature & Time & Repeats \\
\hline Initial denaturation & $95^{\circ} \mathrm{C}$ & $1 \mathrm{~min}$ & 1 \\
\hline Denaturation & $95^{\circ} \mathrm{C}$ & $50 \mathrm{~s}$ & \multirow{2}{*}{20} \\
\hline Annealing & $60^{\circ} \mathrm{C}$ & $1 \mathrm{~min}$ & \\
\hline Elongation & $68^{\circ} \mathrm{C}$ & $6 \mathrm{~min}^{*}$ & \\
\hline Final elongation & $68^{\circ} \mathrm{C}$ & $7 \mathrm{~min}$ & 1 \\
\hline Storage & $4^{\circ} \mathrm{C}$ & $\infty$ & 1 \\
\hline
\end{tabular}

*1 min/kb of plasmid length

After completion of the thermocycler program, the mutagenesis protocol was continued with Dpnl digestion of the amplification products (chapter 2.2.1.4.4). 


\subsection{Mycoplasma contamination PCR}

To detect a possible contamination of eukaryotic cells by mycoplasma, a PCR with mycoplasma specific primers (chapter 2.1.12.4) and the Platinum ${ }^{\circledR}$ Taq DNA Polymerase (Invitrogen) with the supplied reagents was performed. Therefore, $200 \mu \mathrm{l}$ of used cell culture medium was incubated for $5 \mathrm{~min}$ at $95^{\circ} \mathrm{C}$. After mixing and centrifugation at $1500 \times g$ for $1 \mathrm{~min}$ the supernatant was used for reaction mixture. A mycoplasma contaminated cell culture medium was used as positive control.

The following reagents for $50 \mu \mathrm{l}$ of reaction mixture were pipetted into a PCR reaction tube:

\begin{tabular}{l|c}
\hline \multicolumn{1}{c|}{ Component } & \multicolumn{1}{c}{ Volume } \\
\hline Supernatant from cell culture & $1.0 \mu \mathrm{l}$ \\
\hline PCR Buffer $(10 \mathrm{x})$ & $5.0 \mu \mathrm{l}$ \\
\hline $\mathrm{MgCl}_{2}(50 \mathrm{mM})$ & $2.0 \mu \mathrm{l}$ \\
\hline Primer forward $(10 \mathrm{pmol} / \mu \mathrm{l})$ & $0.5 \mu \mathrm{l}$ \\
\hline Primer reverse $(10 \mathrm{pmol} / \mu \mathrm{l})$ & $0.5 \mu \mathrm{l}$ \\
\hline dNTP mix $(10 \mathrm{mM})$ & $1.0 \mu \mathrm{l}$ \\
\hline Platinum Taq DNA Polymerase $(5 \mathrm{U} / \mu \mathrm{l})$ & $0.2 \mu \mathrm{l}$ \\
\hline Ampuwa & $39.8 \mu \mathrm{l}$ \\
\hline
\end{tabular}

The reaction mixture was incubated in a thermocycler using the following PCR program:

\begin{tabular}{l|c|c|c}
\hline \multicolumn{1}{c|}{ Step } & Temperature & Time & Repeats \\
\hline Initial denaturation & $94^{\circ} \mathrm{C}$ & $1 \mathrm{~min}$ & 1 \\
\cline { 1 - 3 } Denaturation & $94^{\circ} \mathrm{C}$ & $30 \mathrm{~s}$ & \multirow{2}{*}{35} \\
\cline { 1 - 2 } Annealing & $55^{\circ} \mathrm{C}$ & $2 \mathrm{~min}$ & \\
\hline Elongation & $72^{\circ} \mathrm{C}$ & $1 \mathrm{~min}$ & 1 \\
\cline { 1 - 3 } Final elongation & $72^{\circ} \mathrm{C}$ & $7 \mathrm{~min}$ & 1 \\
\hline Storage & $4^{\circ} \mathrm{C}$ & $\infty$ & \\
\hline
\end{tabular}

After completion of the thermocycler program, loading buffer was added to $10 \mu \mathrm{l}$ of the reaction mixture and loaded on a 1.5\% agarose gel together with 100 bp DNA Ladder (Invitrogen). 


\subsection{Reverse transcription}

To remove remaining DNA; a DNase digestion (Sigma-Aldrich) prior to transcription of mRNA into cDNA using the SuperScript ${ }^{\circledR}$ II Reverse Transcriptase (Invitrogen) was performed. $5 \mu \mathrm{g}$ of RNA was diluted with DEPC- $\mathrm{H}_{2} \mathrm{O}$ in a total volume of $8 \mu \mathrm{l}$ and further mixed with $1 \mu \mathrm{l}$ of $10 \mathrm{x}$ reaction buffer and $1 \mu \mathrm{l}$ of DNase I by pipetting. After incubation for $15 \mathrm{~min}$ at $\mathrm{RT}$, the reaction was stopped by addition of $1 \mu \mathrm{l}$ Stop Solution, following incubation for $10 \mathrm{~min}$ at $70^{\circ} \mathrm{C}$ and $1 \mathrm{~min}$ on ice. Afterwards, DNase I digested RNA was mixed with $1 \mu \mathrm{l}$ of Random Hexamers (Invitrogen) and $1 \mu \mathrm{l}$ of $10 \mathrm{mM}$ dNTPs and incubated for $5 \mathrm{~min}$ at $65^{\circ} \mathrm{C}$. After incubation for $1 \mathrm{~min}$ on ice, $2 \mu \mathrm{l}$ of $0.1 \mathrm{M} \mathrm{DTT}$ and $4 \mu \mathrm{l}$ of $5 \mathrm{x}$ FirstStrand Buffer was added and mixed by pipetting. After incubation for 2 min at $42^{\circ} \mathrm{C}, 1 \mu \mathrm{l}$ of SuperScript II Reverse Transcriptase was added. The solution was mixed by pipetting and incubated for $50 \mathrm{~min}$ at $42^{\circ} \mathrm{C}$. The reaction was stopped by enzyme inactivation at $70^{\circ} \mathrm{C}$ for $15 \mathrm{~min}$. Amplified cDNA was cooled down for $5 \mathrm{~min}$ on ice and stored at $-20^{\circ} \mathrm{C}$.

The efficiency of cDNA synthesis was controlled by PCR analysis using specific primers for the housekeeping gene mouse Hprt (chapter 2.2.1.2.1.1).

\subsection{Quantitative Real-Time PCR}

To quantify the relative mRNA expression levels of a certain gene of interest a quantitative Real-Time PCR (qRT-PCR) analysis was performed by using fluorescent labeled dNTPs for PCR reactions. After each PCR cycle the fluorescent intensity of labeled double stranded DNA was measured using the 7900HT Fast Real-Time PCR System (Applied Biosystems). During the exponential phase a threshold value of PCR cycles was determined in a $\mathrm{Ct}$ value. This value defines the PCR cycle with the optimal PCR conditions and was used for quantification calculations. The generated data were evaluated with the SDS 2.2.1 software (Applied Biosystems) and transferred to MS Excel (Microsoft) for further calculations using the $\Delta \Delta$-Ct method. The mRNA expression of two housekeeping genes, mouse Hprt and mouse Tbp (TATA box binding protein) were used for normalization.

Prior to the qRT-PCR analysis, the used cDNA was usually diluted 1:10 with Ampuwa. The analysis was carried out in $10 \mu \mathrm{l}$ reactions with $2.5 \mu \mathrm{l}$ of diluted cDNA, $2.5 \mu \mathrm{l}$ of primer solution with forward and reverse primer ( $1 \mathrm{pmol} / \mu \mathrm{l}$ each primer) and $5.0 \mu$ l of QuantiTect ${ }^{\circledR}$ 
SYBR $^{\circledR}$ Green PCR Kit (Qiagen). The reactions were performed in triplicate in a 384-well plate on 7900HT Fast Real-Time PCR System using the following PCR program:

\begin{tabular}{l|c|c|c}
\hline \multicolumn{1}{c|}{ Step } & Temperature & Time & Repeats \\
\hline & $50^{\circ} \mathrm{C}$ & $2 \mathrm{~min}$ & 1 \\
\hline Initial denaturation & $95^{\circ} \mathrm{C}$ & $15 \mathrm{~min}$ & 1 \\
\hline Denaturation & $94^{\circ} \mathrm{C}$ & $15 \mathrm{~s}$ & \multirow{2}{*}{40} \\
\hline Annealing & $60^{\circ} \mathrm{C}$ & $30 \mathrm{~s}$ & \\
\hline \multirow{2}{*}{ Elongation } & $72^{\circ} \mathrm{C}$ & $1 \mathrm{~min}$ & \\
\hline \multirow{2}{*}{ Dissociation curve } & $95^{\circ} \mathrm{C}$ & $15 \mathrm{~s}$ & 1 \\
\cline { 2 - 4 } & $60^{\circ} \mathrm{C}$ & $15 \mathrm{~s}$ & 1 \\
\cline { 2 - 4 } & $95^{\circ} \mathrm{C}$ & $15 \mathrm{~s}$ & 1 \\
\hline
\end{tabular}

\subsection{Sequencing analysis}

The sequencing analysis was performed based on the chain-terminating Sanger sequencing in order to determine the nucleotide sequence of a DNA fragment of interest (Sanger et al., 1977). During the elongation step of the sequence reaction, deoxynucleotides and dideoxynucleotides, which are labeled with different fluorescent dyes, were incorporated into the DNA. The elongation of the chain was terminated selectively at A, C, G or T when dideoxynucleotides were incorporated at the 3' end of the growing chain. Further elongation of the chain was prevented, because the chain lacks a 3'-hydroxyl group once a dideoxynucleotide was incorporated. The nucleotides were determined due to the detected dye and the positions of the nucleotides within the DNA strand were determined due to the length of the fragments.

The generated PCR product or plasmid was purified before sequencing analysis using the ready to use reaction system MSB $^{\circledR}$ Spin PCRapace Kit (Invitek) or the MultiScreen ${ }^{\circledR}$ PCR96 Filter Plates (Merck Millipore). The purification was done according to manufacturer's instructions.

The sequencing analysis was carried out in a $10 \mu$ reaction using the BigDye ${ }^{\circledR}$ Terminator v1.1 Cycle Sequencing Kit (Applied Biosystems).

For sequencing of gDNA isolated from tail biopsy for mouse genotyping, $6 \mu$ l of purified gDNA, $1 \mu \mathrm{l}$ of forward or reverse primer (10 pmol/ $\mu \mathrm{l}), 1 \mu \mathrm{l}$ of BigDye Terminator Ready Reaction Mix and $2 \mu \mathrm{l}$ of BigDye Terminator $5 x$ Sequencing Buffer were pipetted into a PCR reaction tube. 
For sequencing of a generated plasmid, $0.5 \mu \mathrm{l}$ of purified plasmid DNA, $1 \mu \mathrm{l}$ of forward or reverse primer $(10 \mathrm{pmol} / \mu \mathrm{l}), 1 \mu \mathrm{l}$ of BigDye Terminator Ready Reaction Mix, $2 \mu \mathrm{l}$ of BigDye Terminator 5x Sequencing Buffer and $5.5 \mu \mathrm{l}$ of Ampuwa were pipetted into a PCR reaction tube.

For sequencing of a patient sample $5 \mu$ of purified DNA, $1 \mu \mathrm{l}$ of forward or reverse primer (10 pmol/ $\mu \mathrm{l}), 2 \mu \mathrm{l}$ of BigDye Terminator Ready Reaction Mix and $2 \mu \mathrm{l}$ of BigDye Terminator 5x Sequencing Buffer were pipetted into a 96 -well plate.

The reaction mixture was incubated in a thermocycler using the following PCR program:

\begin{tabular}{l|c|c|c}
\hline \multicolumn{1}{c|}{ Step } & Temperature & Time & Repeats \\
\hline Denaturation & $95^{\circ} \mathrm{C}$ & $20 \mathrm{~s}$ & \multirow{2}{*}{30} \\
\cline { 1 - 3 } Annealing & $60^{\circ} \mathrm{C}$ & 2 min & \\
\hline Storage & $8^{\circ} \mathrm{C}$ & $\infty$ & 1 \\
\hline
\end{tabular}

After completion of the thermocycler program each sample was mixed with $10 \mu \mathrm{l}$ of Ampuwa. The final sequencing analysis was performed in house sequencing service (Institute of Human Genetics, Göttingen) using the 3500xL Genetic Analyzer (Applied Biosystems). The generated data were evaluated with the Chromas Lite 2.01 software (Technelysium Pty Ltd.) or the Sequence Pilot 4.3.1 software (JSI medical systems).

\subsubsection{Agarose gel electrophoresis of DNA}

The agarose gel electrophoresis is used to separate DNA fragments within an electric field according to their length.

Depending on the expected fragment size of the DNA fragments, gels with an agarose concentration of $0.8-2.0 \%(\mathrm{w} / \mathrm{v})$ were prepared. The appropriate amount of agarose was dissolved in the appropriate amount of $0.5 x$ Tris-Borate EDTA (TBE) buffer by boiling in a microwave. Either Roti ${ }^{\circledR}$-Safe (Carl Roth) or ethidium bromide (Inno-Train Diagnostik) were added to visualize the DNA later under UV light before the mixture was poured into a horizontal gel chamber. After polymerization of the gel, it was loaded with the samples and an appropriate DNA length standard (chapter 2.1.13) in order to be able to determine the length of the DNA fragments. The separation of the DNA fragments was performed in 
$0.5 x$ TBE buffer by the application of an electric field (100-120 V) and afterwards visualized using an UV transilluminator.

\subsection{Purification of DNA fragments from agarose gels}

The purification of DNA fragments from agarose gels was performed using the ready to use reaction system QIAquick ${ }^{\circledR}$ Gel Extraction Kit (Qiagen, Hilden). After separation of DNA fragments, the DNA fragment of interest was excised from the gel with a sterile scalpel on an UV transilluminator and the gel piece was transferred into a reaction tube and weighed. The 3-fold volume of QG Buffer was added according to the weight of the excised DNA fragment $(1 \mathrm{mg}$ is equivalent to $100 \mu \mathrm{l})$. The mixture was incubated for $10 \mathrm{~min}$ at $50^{\circ} \mathrm{C}$ by shaking and mixing every $2-3 \mathrm{~min}$. After dissolving of the gel piece further purification was performed according to manufacturer's instructions. The DNA was eluted with an appropriate volume of Ampuwa and stored at $-20^{\circ} \mathrm{C}$.

\subsubsection{Cloning techniques}

\subsection{Restriction digestion of plasmid DNA}

Enzymatic cleavage of plasmid DNA was performed with restriction endonucleases derived from the companies Invitrogen and New England Biolabs. Generally, per $1 \mu \mathrm{g}$ DNA 10 units of the enzyme were incubated in $1 x$ appropriate buffer at the required temperature for at least $2 \mathrm{~h}$, preferentially $3 \mathrm{~h}$, in a total volume of 20-50 $\mu \mathrm{l}$ filled up with Ampuwa. The complete digestion of the plasmid DNA was checked by agarose gel electrophoresis (chapter 2.2.1.3).

If for the restriction digestion two different enzymes had to be used, either a buffer compatible for both enzymes was chosen or a sequential restriction digestion was performed. For sequential restriction digestion, the digested DNA had to be precipitated after the use of the first restriction enzyme. Therefore, the reaction volume was filled up with Ampuwa to a total volume of $100 \mu \mathrm{l} .10 \mu \mathrm{l}$ of $3 \mathrm{M}$ sodium acetate ( $\mathrm{pH}$ 5.2) was added and the solution was mixed for $12 \mathrm{~s}$. After addition of $300 \mu \mathrm{l} 100 \%$ ethanol (chilled at $-20^{\circ} \mathrm{C}$ ), the solution was mixed by inverting and incubated either for $5 \mathrm{~min}$ at RT or overnight at $-20^{\circ} \mathrm{C}$. The precipitated DNA was centrifuged at $16,000 \times g$ for $10 \mathrm{~min}$ at RT 
and the received DNA pellet was washed with $1 \mathrm{ml}$ of $70 \%$ ethanol (chilled at $-20^{\circ} \mathrm{C}$ ). After centrifugation at $16,000 \times g$ for $5 \mathrm{~min}$ at $\mathrm{RT}$, the pellet was dried at $56^{\circ} \mathrm{C}$ and resolved in an appropriate volume of Ampuwa by shaking for $10 \mathrm{~min}$ at $56^{\circ} \mathrm{C}$. Afterwards, restriction digestion with the second restriction enzyme was performed.

\subsection{Cloning of DNA fragments using In-Fusion ${ }^{\circledR}$ HD Cloning Kit}

For cloning of DNA fragments into vectors the In-Fusion ${ }^{\circledR}$ HD Cloning Kit (Clontech) was used according to manufacturer's instructions. The gene of interest was amplified by PCR using gene-specific In-Fusion primers. For the In-Fusion cloning reaction the linearized vector, the purified PCR fragment and $2 \mu$ of $5 x$ In-Fusion HD Enzyme Premix were adjusted with Ampuwa to a total reaction volume of $10 \mu \mathrm{l}$. The reaction mixture was incubated for $15 \mathrm{~min}$ at $37^{\circ} \mathrm{C}$ followed by an additional incubation for $15 \mathrm{~min}$ at $50^{\circ} \mathrm{C}$. After addition of $40 \mu \mathrm{l}$ TE buffer (Invitrogen), E. coli DH5a competent cells were transformed with $2.5 \mu \mathrm{l}$ of the reaction mixture (chapter 2.2.1.4.3).

\subsection{Transformation of competent cells}

For transformation of competent cells with the In-Fusion reaction mixture, $50 \mu \mathrm{l}$ of $E$. coli DH5a competent cells (Invitrogen) was thawed on ice and $2.5 \mu$ of the reaction mixture was added. The mixture was incubated for $30 \mathrm{~min}$ on ice and after a heat shock for $45 \mathrm{~s}$ at $42^{\circ} \mathrm{C}$ placed for $2 \mathrm{~min}$ on ice. Afterwards, $450 \mu \mathrm{l}$ of S.O.C. medium (Invitrogen) was added and the bacteria were incubated for $1 \mathrm{~h}$ at $37^{\circ} \mathrm{C}$ by slight shaking. $50 \mu \mathrm{l}$ and $100 \mu \mathrm{l}$ of the transformed bacteria were separately spread on LB agar plates containing an antibiotic appropriate for the cloning vector and incubated at $37^{\circ} \mathrm{C}$ overnight.

For transformation of competent cells with Dpnl-treated mutagenesis reaction mixture, $100 \mu \mathrm{l}$ of $E$. coli DH5a competent cells was thawed on ice and $5 \mu \mathrm{l}$ of the reaction mixture was added. After incubating the mixture for 30 min on ice, a heat shock was performed for $45 \mathrm{~s}$ at $42^{\circ} \mathrm{C}$. The mixture was further placed for $2 \mathrm{~min}$ on ice and $700 \mu \mathrm{l}$ of S.O.C. medium was added. By slight shaking, the bacteria were incubated for $1 \mathrm{~h}$ at $37^{\circ} \mathrm{C}$. On LB agar plates containing an antibiotic appropriate for the cloning vector, $250 \mu \mathrm{l}$ and $550 \mu \mathrm{l}$ of the transformed bacteria were spread separately. The agar plates were incubated at $37^{\circ} \mathrm{C}$ overnight. 


\subsection{Mutagenesis}

The QuikChange II XL Site-Directed Mutagenesis Kit (Agilent Technologies) was used to create specific, targeted changes in double stranded plasmid DNA. PCR reaction using PfuUltra High-Fidelity DNA Polymerase and specific primers were performed (chapter 2.2.1.2.1.4). The amplification products were digested by addition of $1 \mu \mathrm{l} D \mathrm{p}$ l restriction enzyme $(10 \mathrm{U} / \mu \mathrm{l})$ directly to each amplification reaction, following incubation for $1 \mathrm{~h}$ at $37^{\circ} \mathrm{C}$ and transformation into E. coli DH5a competent cells (chapter 2.2.1.4.3). The next day, minipreparation of plasmid DNA (chapter 2.2.1.1.2) was performed followed by test restriction digestion of the isolated plasmid DNA (chapter 2.2.1.4.1) using appropriate restriction enzymes to identify positive clones. Plasmid DNA was purified with MSB Spin PCRapace Kit and the correct nucleotide sequences of the generated plasmids were validated by sequencing analysis (chapter 2.2.1.2.4).

\subsubsection{Yeast two-hybrid assay}

The yeast two-hybrid assay represents an in vivo method to identify novel protein interactions, confirm putative direct interactions and define interacting domains using yeast cells. The Matchmaker ${ }^{\circledR}$ Gold Yeast two-Hybrid System (Clontech) and the yeast strain Y2HGold (Clontech) were used.

$50 \mathrm{ml}$ of YPDA medium was inoculated with 3-4 yeast colonies from an YPDA agar plate and incubated $(16-18 \mathrm{~h})$ at $30^{\circ} \mathrm{C}$ by shaking at $220 \mathrm{rpm}$ overnight. $4-8 \mathrm{ml}$ of the overnight culture $\left(\mathrm{OD}_{600}>1.5\right)$ was transferred into $300 \mathrm{ml}$ of YPDA medium $\left(\mathrm{OD}_{600} \sim 0.3\right)$ and incubated for $3-4 \mathrm{~h}$ at $30^{\circ} \mathrm{C}$ by shaking at $220 \mathrm{rpm}$ until an $\mathrm{OD}_{600}$ between $0.4-0.6$ was reached. The yeast cell suspension was portioned into $50 \mathrm{ml}$ falcons and centrifuged at $1000 \times g$ for $5 \mathrm{~min}$ at RT. Meanwhile, Yeastmaker ${ }^{\mathrm{TM}}$ Carrier DNA (10 mg/ml) (Clontech) was denatured for $10 \mathrm{~min}$ at $95^{\circ} \mathrm{C}$ and placed for $5 \mathrm{~min}$ on ice. Each yeast cell pellet was resuspended in $30 \mathrm{ml}$ Ampuwa and centrifuged at $1000 \times \mathrm{g}$ for $5 \mathrm{~min}$ at RT. Thereafter, each yeast cell pellet was resuspended in $1.5 \mathrm{ml}$ of freshly prepared TE/LiAc solution. $10 \mu \mathrm{l}$ of denatured Carrier DNA was added to $1 \mu \mathrm{g}$ of each plasmid and mixed by pipetting. After addition of $100 \mu \mathrm{l}$ yeast cell solution and mixing, $600 \mu \mathrm{l}$ of freshly prepared PEG/LiAc solution was added and mixed. The reaction mixture was incubated for $30 \mathrm{~min}$ at $30^{\circ} \mathrm{C}$ by shaking at $500 \mathrm{rpm}$. Afterwards, $70 \mu \mathrm{l}$ of DMSO solution was added and mixed by inverting. Next, heat shock for $15 \mathrm{~min}$ at $42^{\circ} \mathrm{C}$ was performed, followed by incubation 
for $2 \mathrm{~min}$ on ice and centrifugation at $16,000 \times g$ for $1 \mathrm{~min}$. Each yeast cell pellet was resuspended in $300 \mu \mathrm{l}$ of Ampuwa and plated as drops of $10 \mu \mathrm{l}$ each on -LT and -LTHA agar plates, respectively. The agar plates were incubated for $5-7 \mathrm{~d}$ at $30^{\circ} \mathrm{C}$.

\subsubsection{Protein manipulation methods}

\subsubsection{Protein isolation from eukaryotic cells}

For protein isolation, eukaryotic cells were harvested from cell culture vessel using an appropriate protocol (chapter 2.2.4.2). Nuclear and cytoplasmic protein extracts were isolated using the NE-PER ${ }^{\circledR}$ Nuclear and Cytoplasmic Extraction Reagents Kit (Thermo Scientific) according to manufacturer's instructions.

\subsubsection{Protein isolation from yeast cells}

For protein isolation from yeast cells, $8 \mathrm{ml}$ of SD medium with addition of adenine and histidine (chapter 2.1.10.1) was inoculated with a single yeast colony from a -LT agar plate and incubated $(16-18 \mathrm{~h})$ at $30^{\circ} \mathrm{C}$ by shaking at $250 \mathrm{rpm}$ overnight. The overnight culture $\left(\mathrm{OD}_{600} \sim 1.0\right)$ was centrifuged at $1000 \times \mathrm{g}$ for $5 \mathrm{~min}$ at $\mathrm{RT}$. The yeast cell pellet was resuspended in $500 \mu \mathrm{l}$ of $2 \mathrm{M}$ chilled LiAc and incubated for $5 \mathrm{~min}$ on ice. After centrifugation at $1000 \times g$ for $5 \mathrm{~min}$ at RT, the cell pellet was resuspended in $100 \mu \mathrm{l}$ of $0.4 \mathrm{M}$ chilled $\mathrm{NaOH}$, incubated for $5 \mathrm{~min}$ on ice and centrifuged at $1000 \times \mathrm{g}$ for $5 \mathrm{~min}$. The cell pellet was resuspended in $100 \mu \mathrm{l}$ of sample buffer containing $25 \mu \mathrm{l}$ of NuPAGE ${ }^{\circledR}$ LDS Sample Buffer (4x) (Invitrogen), $10 \mu \mathrm{l}$ of $1 \mathrm{M}$ DTT and $65 \mu \mathrm{l}$ of Ampuwa. After yeast proteins were denatured for $10 \mathrm{~min}$ at $70^{\circ} \mathrm{C}$, the mixture was placed for $5 \mathrm{~min}$ on ice and was further centrifuged at $16,000 \times g$ for $1 \mathrm{~min}$ to pellet cell debris. The protein containing supernatant was transferred into a new reaction tube and $28 \mu \mathrm{l}$ of the supernatant was used for SDS-PAGE (chapter 2.2.2.6). 


\subsubsection{Protein concentration from cell culture medium}

Proteins from cell culture medium were concentrated using the Vivaspin ${ }^{\circledR}$ Turbo 15 centrifugal concentrators (30 kDa MWCO) (Sartorius) according to manufacturer's instructions.

\subsubsection{Determination of protein concentration}

Protein concentration was determined according to the method of Bradford (1976). The used solution Roti ${ }^{\circledR}$-Nanoquant (5x) (Carl Roth) consists of the Coomassie Brilliant Blue dye that has the property to bind unspecifically to proteins in acidic solution, resulting in a shift of its absorption maximum from $465 \mathrm{~nm}$ to $595 \mathrm{~nm}$.

A Bovine Serum Albumin Standard Ampule (Thermo Scientific) was diluted to generate standard protein concentrations for a calibration curve to determine the protein concentration by extrapolating to this curve.

To determine protein concentration $5 x$ Roti-Nanoquant was diluted with $\mathrm{dd}_{2} \mathrm{O}$ to a final $1 \mathrm{x}$ concentration and further stored at $4^{\circ} \mathrm{C}$ up to 3 month. Protein sample was diluted 1:100 with Ampuwa and $50 \mu \mathrm{l}$ of the sample was pipetted into a 96-well plate in triplicate. Ampuwa was used as control. Each diluted protein sample was mixed with $200 \mu$ of 1x Roti-Nanoquant and incubated for $5 \mathrm{~min}$ at RT. The absorptions were measured using the Synergy ${ }^{\mathrm{TM}} \mathrm{Mx}$ plate reader (BioTek) and calculated with the provided Gen5 ${ }^{\mathrm{TM}}$ software.

\subsubsection{Co-immunoprecipitation}

Co-immunoprecipitation (Co-IP) is a technique to identify protein-protein interactions by using a target protein antibody for the indirect capture of proteins that are bound to the target protein. These protein complexes can be further analyzed for example by western blot analysis to identify unknown complex members.

To pre-clear isolated protein extract of proteins which can bind unspecifically to the beads, 500-1000 $\mu \mathrm{g}$ of protein extract and $20 \mu \mathrm{l}$ of Protein G Magnetic Beads (New England Biolabs) were gently mixed, filled up with lysis buffer (chapter 2.1.6) to a total volume of $500 \mu \mathrm{l}$ and rotated on the Stuart ${ }^{\circledR} \mathrm{SB} 2$ rotator (Bibby Scientific) for $1 \mathrm{~h}$ at $4^{\circ} \mathrm{C}$. Afterwards, a magnetic field was applied for 1 min by placing the reaction tube into a 6-Tube Magnetic 
Separation Rack (New England Biolabs) to pull the beads to the side of the tube. The supernatant was pipetted into a new reaction tube, $2 \mu \mathrm{g}$ of appropriate antibody (chapter 2.1.11.1) was added and the mixture was incubated on the rotator at $4^{\circ} \mathrm{C}$ overnight. No antibody was added to the negative control. The following day, $20 \mu \mathrm{l}$ of Protein $\mathrm{G}$ Magnetic Beads was added and the mixture was incubated on the rotator for $2 \mathrm{~h}$ at $4^{\circ} \mathrm{C}$. After brief centrifugation, a magnetic field was applied for $1 \mathrm{~min}$ and the supernatant was removed. The beads were washed with $500 \mu$ l of lysis buffer and incubated on the rotator for $5 \mathrm{~min}$ at $4^{\circ} \mathrm{C}$. The mixture was briefly centrifuged and the washing step was additionally repeated 4 times as previously described. For the final washing step, $500 \mu \mathrm{l}$ of TBS buffer was used. Thereafter, a magnetic field was applied for $1 \mathrm{~min}$, the supernatant was removed and the bead pellet was gently mixed with $16.3 \mu \mathrm{l}$ of lysis buffer, $6.25 \mu \mathrm{l}$ of LDS Sample Buffer $(4 \mathrm{x})$ and $2.5 \mu \mathrm{l}$ of $1 \mathrm{M}$ DTT. Proteins were denatured for $10 \mathrm{~min}$ at $70^{\circ} \mathrm{C}$ and separated from the beads meanwhile. The mixture was placed for $5 \mathrm{~min}$ on ice and after applying a magnetic field for $1 \mathrm{~min}$, the supernatant was completely used for SDS-PAGE (chapter 2.2.2.6).

\subsubsection{SDS polyacrylamide gel electrophoresis}

SDS polyacrylamide gel electrophoresis (SDS-PAGE) was used for the separation of proteins according to their molecular weight based on the method described by (Laemmli, 1970). The method bases on the use of gels and buffers with an operating $\mathrm{pH}$ of 7.0. This neutral $\mathrm{pH}$ increases the stability of both proteins and gels and leads to a better result of electrophoresis.

In general, samples consisted of $30 \mu \mathrm{g}$ protein, 0.25 volume LDS Sample Buffer (4x) and $10 \% 1 \mathrm{M}$ DTT (reducing agent), filled up with Ampuwa to a total volume of $20-28 \mu \mathrm{l}$, depending on the maximum sample loading volume of the gel. Proteins were denatured for $10 \mathrm{~min}$ at $70^{\circ} \mathrm{C}$ and placed on ice for $5 \mathrm{~min}$. After brief centrifugation, protein samples were loaded onto a gradient gel (NuPAGE ${ }^{\circledR} 3-8 \%$ Tris-Acetate Gel or NuPAGE ${ }^{\circledR} 4-12 \%$ Bis-Tris Gel, Invitrogen) placed in Novex ${ }^{\circledR}$ Mini-Cell Electrophoresis System (Invitrogen). Pre-stained molecular weight standard $\left(10 \mu \mathrm{l}\right.$ of SeeBlue ${ }^{\circledR}$ Plus2 Pre-Stained Protein Standard or $20 \mu \mathrm{l}$ of HiMark ${ }^{\mathrm{TM}}$ Pre-Stained High Molecular Weight Protein Standard, Invitrogen) was additionally loaded on the gel for determination the size of the separated proteins. Gel electrophoresis was performed for $10 \mathrm{~min}$ at $80 \mathrm{~V}$ and further at either $120 \mathrm{~V}$ (10-well gel) or $160 \mathrm{~V}$ (20-well gel) and depending on the composition of the gel, in 
1x NuPAGE ${ }^{\circledR}$ Tris-Acetate SDS Running Buffer or $1 \times$ NuPAGE $^{\circledR}$ MES SDS Running Buffer (Invitrogen).

\subsubsection{Transfer of proteins from a polyacrylamide gel to a membrane}

For detection of proteins by western blot analysis, separated proteins from a polyacrylamide gel were transferred to a membrane based on the method described by (Gershoni and Palade, 1983). Proteins $<150 \mathrm{kDa}$ were transferred on a nitrocellulose membrane (Amersham ${ }^{\mathrm{TM}}$ Protran $^{\mathrm{TM}}$ Supported $0.45 \mu \mathrm{m}$ NC, GE Healthcare), whereas proteins $>150 \mathrm{kDa}$ were transferred on a PVDF membrane (Amersham ${ }^{\mathrm{TM}}$ Hybond $^{\mathrm{TM}}$-P PVDF Transfer Membrane $0.45 \mu \mathrm{m}$, GE Healthcare).

One membrane and four Blotting Paper Sheets (Sartorius) were cut to the size of the gradient gel. The nitrocellulose membrane was activated in $\mathrm{dd}_{2} \mathrm{O}$ and further equilibrated in chilled transfer buffer I (chapter 2.1.6), while the PVDF membrane was activated in $100 \%$ methanol for approximately $30 \mathrm{~s}$ and then equilibrated in chilled transfer buffer II (chapter 2.1.6) for $5 \mathrm{~min}$. Blotting papers and foam pads were soaked in appropriate transfer buffer. For transfer, the tank blotting system Mini Protean ${ }^{\circledR}$ || Cell (Bio-Rad) was used and the components were placed in a provided cassette on top of each other as follows: two sheets of blotting paper, membrane, polyacrylamide gel and two sheets of blotting paper. Potential air bubbles were carefully removed. The cassette was inserted into the tank in the way that the gel was located near to the cathode of the blotting system. The tank further contained appropriate and chilled transfer buffer and a cooling unit. The transfer of proteins $<150 \mathrm{kDa}$ was performed at $250 \mathrm{~mA}$ per gel for $1 \mathrm{~h}$ at $4^{\circ} \mathrm{C}$. The transfer of proteins $>150 \mathrm{kDa}$ was performed at $25 \mathrm{~V}$ overnight. Additionally, the buffer tank was put in an ice box and the buffer was agitated by a magnetic stirrer to avoid the formation of an ion gradient and to insure homogeneity of the buffer temperature.

\subsubsection{Protein detection on membranes using antibodies}

After protein transfer to a membrane, unspecific binding sites were blocked by incubating the membrane, depending on the used antibody, with blocking solution I or II (chapter 2.1.6) for at least $1 \mathrm{~h}$ at RT. Primary antibody (chapter 2.1.11.1) was diluted, depending on the antibody, in antibody diluent solution I or II (chapter 2.1.6) at $4{ }^{\circ} \mathrm{C}$ overnight. The 
following day, the membrane was washed with TBST buffer three times for $10 \mathrm{~min}$ to remove unbound antibody. The membrane was further incubated with appropriate secondary antibody (chapter 2.1.11.2) diluted, depending on the antibody, in antibody diluent solution I or II for 1.5-2 $\mathrm{h}$ at RT. To remove unbound antibody, the membrane was washed three times for $10 \mathrm{~min}$ with TBST buffer. The Immobilon $^{\mathrm{TM}}$ Western Chemiluminescent HRP Substrate (Merck Millipore) was used to visualize membrane bound proteins and was prepared according to manufacturer's instructions. The membrane was covered with the prepared solution, placed between a plastic wrap and incubated for $5 \mathrm{~min}$ at RT. The chemiluminescent signals were digitally detected using FluorChem ${ }^{\circledR} \mathrm{Q}$ (Alpha Innotech) and evaluated with the provided AlphaView ${ }^{\circledR} \mathrm{Q}$ software (Alpha Innotech).

\subsubsection{Histological methods}

\subsubsection{Dissection of mouse tissues and embryos}

For dissection of mouse tissues, the mouse was killed by $\mathrm{CO}_{2}$-asphyxation and the required tissues were dissected. For RNA isolation (chapter 2.2.1.1.5) the tissues were immediately frozen in liquid nitrogen and further stored at $-80^{\circ} \mathrm{C}$.

For dissection of mouse embryos, one-to-one matings of wildtype Whirligig $\left(\mathrm{Chd} 7^{+/+}\right.$) female and wildtype Whirligig $\left(\mathrm{Chd}^{+/+}\right)$male mice were set up. Female Whirligig mice were daily checked for a vaginal plug, to determine the age of the embryos. When a vaginal plug was observed, the day was counted as embryonic stage 0.5 (E0.5). At the desired embryonic stage, the pregnant Whirligig mouse was killed by cervical dislocation and the uterus was removed. The embryos were dissected out of the yolk sacs, a tail biopsy was performed for gDNA isolation (chapter 2.2.1.1.1), further genotyping PCR (chapter 2.2.1.2.1.3) and sequencing analysis (chapter 2.2.1.2.4) to confirm the genotype. The embryos were immediately frozen in liquid nitrogen and further stored at $-80^{\circ} \mathrm{C}$ for RNA isolation (chapter 2.2.1.1.5). 


\subsubsection{Fixation of cells on slides}

For histological analysis, 09-1 cells were grown on culture slides (BD Biosciences) providing eight chambers. Before plating the cells, slides were coated with matrigel (chapter 2.2.4.1.2).

Before further histological analysis, cells were washed two to three times with DPBS and fixed with $4 \%$ PFA for 10 min at RT.

\subsubsection{Immunofluorescence staining}

Before immunofluorescence staining, 09-1 cells were fixed (chapter 2.2.3.2) and further washed two to three times with ice cold DPBS. Cells were permeabilized with $0.1 \%$ Triton X-100 solution for $10 \mathrm{~min}$. After washing the cells with DBPS three times for $5 \mathrm{~min}$, unspecific binding sites were blocked by incubation with blocking solution (chapter 2.1.6) for at least $1 \mathrm{~h}$ at RT. Cells were incubated with the appropriate primary antibody (chapter 2.1.11.1) diluted in antibody diluent solution (chapter 2.1.6) in a humidified chamber at $4^{\circ} \mathrm{C}$ overnight. The following day, cells were washed with DPBS three times for $10 \mathrm{~min}$ and incubated with the appropriate secondary antibody (chapter 2.1.11.2) diluted in antibody diluent solution (chapter 2.1.6) in a humidified chamber for 1.5-2 $\mathrm{h}$ at RT. Afterwards, cells were washed with DPBS three times for $10 \mathrm{~min}$ and shortly air-dried. Cells were mounted with Fluoroshield ${ }^{\mathrm{TM}}$ with Dapi (Sigma-Aldrich), sealed with nail polish and stored in the dark at $4^{\circ} \mathrm{C}$. Immunofluorescence staining was analyzed with the confocal laser scanning microscope IX81 (Olympus) using the provided FV10-ASW1.7 software.

\subsubsection{Cell culture methods}

\subsubsection{Coating of cell culture vessels}

\subsection{Preparation of fibronectin coated cell culture vessels}

Human fibronectin (Merck Millipore) was used for the preparation of fibronectin coated cell culture vessels. The fibronectin was diluted in chilled DPBS to a concentration of $50 \mu \mathrm{g} / \mathrm{ml}$. One cell culture plate $(\varnothing 10 \mathrm{~cm}$ ) was coated with $5 \mathrm{ml}$ fibronectin solution and 
left on the culture vessel for at least $10 \mathrm{~min}$. Then, the solution was aspirated and could be reused several times when stored at $4^{\circ} \mathrm{C}$. The culture vessel was left open until it got dry (approximately $10 \mathrm{~min}$ ) and afterwards the cells were plated on the coated culture vessel.

\subsection{Preparation of matrigel coated cell culture vessels}

For the preparation of matrigel coated cell culture vessels the Matrigel ${ }^{\circledR}$ Basement Membrane Matrix (Corning) was used. During the work with matrigel, chilled pipette tips and DPBS were used. After thawing on ice at $4^{\circ} \mathrm{C}$, aliquots of $100 \mu$ l were prepared on ice and further stored at $-20^{\circ} \mathrm{C}$. Before use, required quantity of aliquots was thawed on ice at $4^{\circ} \mathrm{C}$ either at least for $1 \mathrm{~h}$ or overnight. One aliquot was diluted 1:50 in DPBS, respectively. To coat one cell culture plate $(\varnothing 10 \mathrm{~cm}), 5 \mathrm{ml}$ of matrigel solution was used and left on the culture vessel for at least $1 \mathrm{~h}$ at RT before use. In advance coated culture vessels were stored up to $5-6 \mathrm{~d}$ at $4^{\circ} \mathrm{C}$. The matrigel solution was carefully aspirated just before plating the cells.

\subsubsection{Culture of eukaryotic cells}

\subsection{Culture and passaging of murine JoMa1 and JoMa1.3 cells}

Murine JoMa1 and JoMa1.3 cells (chapter 2.1.9) were cultured for 3-4 d on fibronectin coated cell culture vessels (chapter 2.2.4.1.1) until they reached a confluence of approximately $80 \%$. The NCC medium (chapter 2.1 .9 .1 ) was carefully aspirated and the cells were washed with DPBS. The DPBS was removed from the cells and 1-4 $\mathrm{ml}$ of Trypsin-EDTA (PAN-Biotech), depending on the size of the culture vessel, was applied. After incubation for $3-5 \mathrm{~min}$ at $37^{\circ} \mathrm{C}$ and $5 \% \mathrm{CO}_{2}$ in a humidified incubator, the detachment of the cells from the culture vessel was controlled under the light microscope. To inactivate the Trypsin-EDTA treatment, an equal volume of Trypsin Inhibitor (SigmaAldrich) was added and the cell suspension was centrifuged at $150 \times g$ for $5 \mathrm{~min}$. The supernatant was discarded and the cell pellet was resuspended in NCC medium. The cells were generally split 1:10 and plated on fibronectin coated culture vessels in an appropriate volume of NCC medium. The cells were cultivated at $37^{\circ} \mathrm{C}$ in a $5 \% \mathrm{CO}_{2}$ 
humidified incubator. The medium was daily replaced with freshly added 4-OHT to remove not adherent cells and in order to keep the cells in their proliferating and undifferentiated state.

\subsection{Culture and passaging of murine 09-1 cells}

Until the murine 09-1 cells (chapter 2.1.9) reached a confluence of approximately $80 \%$ they were cultured for 3-4 $d$ on matrigel coated cell culture vessels (chapter 2.2.4.1.2). After the aspiration of the ESC medium (chapter 2.1.9.1), the cells were washed with DPBS that was removed afterwards. Depending on the size of the culture vessel, the cells were treated with 1-4 ml Trypsin-EDTA (PAN-Biotech) and incubated in a $37^{\circ} \mathrm{C}$ and $5 \% \mathrm{CO}_{2}$ humidified incubator for 3-5 min. Under the light microscope the detachment of the cells was controlled. By addition of an equal volume of ESC medium, the TrypsinEDTA treatment was stopped and the cell suspension was centrifuged at $300 \times g$ for 3 min. After discarding the supernatant, the cell pellet was resuspended in ESC medium and the cells were typically split 1:100. The cells were plated in ESC medium on matrigel coated cell culture vessels and cultivated at $37^{\circ} \mathrm{C}$ in a $5 \% \mathrm{CO}_{2}$ humidified incubator. The next day, the medium was replaced to remove not adherent cells and changed every 2-3 d thereafter.

\subsection{Culture and passaging of human HEK293 cells}

The human HEK293 cells (chapter 2.1.9) were cultured for 3-4 d in cell culture flasks until they reached a confluence of approximately $80 \%$. The FB medium (chapter 2.1.9.1) was aspirated and the cells were washed with DPBS. After aspiration of the DPBS, the cells were detached from the culture vessel using 1-4 $\mathrm{ml}^{\text {TrypLE }}{ }^{\mathrm{TM}}$ Express (Gibco), depending on the size of the culture vessel. The detachment of the cells was controlled under the light microscope after incubation in a $37^{\circ} \mathrm{C}$ and $5 \% \mathrm{CO}_{2}$ humidified incubator. To inactivate the TrypLE ${ }^{\mathrm{TM}}$ Express treatment, an equal volume of FB medium was added. The cell suspension was centrifuged at $190 \times \mathrm{g}$ for $5 \mathrm{~min}$. The supernatant was discarded and the cell pellet resuspended in FB medium. A typically split ratio was 1:4 to 1:6. The cells were plated in culture flasks and cultivated at $37^{\circ} \mathrm{C}$ in a $5 \% \mathrm{CO}_{2}$ humidified incubator. 
To remove not adherent cells, the medium was changed the next day and then replaced every $2-3 \mathrm{~d}$.

\subsection{Cryopreservation and thawing of eukaryotic cells}

Cells were cryopreserved from confluent cell culture vessels. JoMa1 or JoMa1.3 cell pellet was resuspended in $1 \mathrm{ml}$ chilled ready-to-use Cryo-SFM (PromoCell) medium. O9-1 cell pellet was resuspended in $1 \mathrm{mI}$ NCC medium with 10\% DMSO (chapter 2.1.9.2), whereas HEK293 cell pellet was resuspended in $500 \mu \mathrm{l}$ of FB medium (chapter 2.1.9.1) and subsequently mixed with $500 \mu \mathrm{l}$ of FB freezing medium (chapter 2.1.9.2). The appropriate mixture was transferred into well labeled cryovials, which were immediately placed in a Mr. Frosty ${ }^{\mathrm{TM}}$ Freezing Container (Kisker) and then frozen at $-80^{\circ} \mathrm{C}$. For long term storage the cryovials were placed in liquid nitrogen.

For the procedure of thawing the cryovial was incubated in a $37^{\circ} \mathrm{C}$ water bath until the cells were almost completely thawed. The cells were carefully mixed with a larger volume of appropriate culture medium (chapter 2.1.9.1) and then centrifuged using appropriate conditions. After removal of the supernatant, the cells were resuspended in culture medium, seeded on appropriate cell culture vessel and cultivated at $37^{\circ} \mathrm{C}$ in a $5 \% \mathrm{CO}_{2}$ humidified incubator. The next day, not adherent cells were removed by changing the culture medium. The further cell culture procedure was performed according to the appropriate cell line as described above.

\subsubsection{Transfection of eukaryotic cells}

\subsection{Transfection of plasmids into eukaryotic cells}

Eukaryotic cells were transfected with plasmids in order to induce a protein overexpression. Lipofectamine ${ }^{\circledR} 2000$ Transfection Reagent (Invitrogen) was used as transfection reagent. This transfection reagent contains lipids which form vesicles with a bilayer sheet, so called liposomes, in aqueous solution. Liposomes and nucleic acids can form nucleic acid lipid complexes which eukaryotic cells can actively take up by endocytosis. The nucleic acids are able to enter the nucleus when the nuclear membrane 
dissolves during mitosis. For efficient transfection, the division rate of the cells must be high as possible.

One day before transfection, cells were plated on cell culture plates or in cell culture flasks. In general, $1.2 \times 10^{6} 09-1$ cells $/ \mathrm{ml}$ were plated on culture plates $(\varnothing 10 \mathrm{~cm})$ and $5.0 \times 10^{6} \mathrm{HEK} 293 \mathrm{cells} / \mathrm{ml}$ were plated in culture flasks $\left(75 \mathrm{~cm}^{2}\right)$. The following day, $30 \mu \mathrm{g}$ of plasmid DNA was gently mixed with Opti-MEM ${ }^{\circledR}$ I Reduced Serum Medium (Gibco) in a total volume of $1875 \mu \mathrm{l}$ and incubated for $5 \mathrm{~min}$ at RT. Furthermore, $75 \mu \mathrm{l}$ of Lipofectamine 2000 was also gently mixed with $1800 \mu \mathrm{l}$ of Opti-MEM and incubated for 5 min at RT. Afterwards, the plasmid DNA and the Lipofectamine 2000 mixture were combined, gently mixed and incubated for $25 \mathrm{~min}$ at RT. Before the transfection mixture was added to the cells, the culture medium was aspirated and the cells were washed with DPBS. $9 \mathrm{ml}$ of ESC medium (chapter 2.1.9.1) was used as culture medium for 09-1 cells. As culture medium for HEK293 cells, $9 \mathrm{ml}$ of DMEM (PAN-Biotech) with 1x NEAA (Gibco) was used. The transfection mixture was applied dropwise to the cells, gently mixed with the culture medium and incubated for $24 \mathrm{~h}$ at $37^{\circ} \mathrm{C}$ in a $5 \% \mathrm{CO}_{2}$ humidified incubator. After 4-5 h, $9 \mathrm{ml}$ of culture medium composed of DMEM with $20 \%$ FBS (PAN-Biotech) and $1 \mathrm{x}$ NEAA was added to the transfection reagent containing culture medium of HEK293 cells. After incubation time, protein was isolated from the transfected cells.

\subsection{Transfection of short interfering RNAs}

Short interfering RNAs (siRNAs) are used for silencing of a specific gene. Thereby, siRNA binds to a specific mRNA and marks it for degradation. This results in a reduced expression of the specific gene. Three different mouse siRNAs against Chd7 (s232009, s232010, s232011, Silencer ${ }^{\circledR}$ Select Pre-Designed \& Validated siRNA, ambion ${ }^{\circledR}$, Life Technologies, Darmstadt) were used. A siRNA with a sequence that do not target any gene product was used as control siRNA (Silencer ${ }^{\circledR}$ Select Negative Control No. 1 siRNA, ambion $^{\circledR}$, Life Technologies, Darmstadt). Lipofectamine 2000 was used as transfection reagent. Thereby, complexes of lipids and siRNA oligonucleotides can be formed which facilitate the uptake of the siRNA molecules into the cells.

One day before transfection, murine 09-1 cells were plated on matrigel coated 6-well cell culture plates. Per well $0.15 \times 10^{6}$ cells were plated in $2.5 \mathrm{ml}$ of ESC medium (chapter 2.1.9.1). The following day, $6 \mu \mathrm{l}$ of siRNA (stock concentration: $10 \mu \mathrm{M}$ ) was gently mixed with $294 \mu \mathrm{l}$ of Opti-MEM and incubated for $5 \mathrm{~min}$ at RT. Furthermore, $12 \mu \mathrm{l}$ of 
Lipofectamine 2000 was also gently mixed with $288 \mu \mathrm{l}$ of Opti-MEM and incubated for 5 min at RT. Afterwards, the siRNA and the Lipofectamine 2000 mixture were combined, gently mixed and incubated for $25 \mathrm{~min}$ at RT. In the meantime, the cells were washed with DPBS and $2.0 \mathrm{ml}$ of ESC medium was added to each well. $500 \mu \mathrm{l}$ of the transfection mixture was applied dropwise to the cells and gently mixed with the culture medium. Each well contained a final siRNA concentration of $20 \mathrm{nM}$. The cells were incubated for $48 \mathrm{~h}$ at $37^{\circ} \mathrm{C}$ in a $5 \% \mathrm{CO}_{2}$ humidified incubator. After incubation time, RNA was isolated from the transfected cells. 


\section{$3 \quad$ Results}

\subsection{Characterization of murine NCC lines}

To choose a suitable cell line for in vitro analyses of NCCs, which are the affected cell type leading to the phenotype seen in CHARGE syndrome patients, the expression pattern of different molecular markers in the two murine cell lines JoMa and O9-1 were examined. For testing the NCC behavior of the different cell lines, the NCC marker genes Sox10 (SRY (sex determining region Y)-box 10), Ngfr (nerve growth factor receptor (TNFR superfamily, member 16)), Snai2 (snail family zinc finger 2), Pax3 (paired box 3), Snai1 (snail family zinc finger 1) and Twist1 (twist basic helix-loop-helix transcription factor 1) were used. Furthermore, the expression of the neuronal marker Nefh (neurofilament, heavy polypeptide), the glia marker Gfap (glial fibrillary acidic protein) and the two smooth muscle markers Cnn1 (calponin 1) and Actg2 (actin, gamma 2, smooth muscle enteric) were investigated. The expression of the melanocyte marker Tyr (tyrosinase) and the chondrocyte marker Col2a1 (collagen, type II, alpha 1) was also analyzed.

\subsubsection{Molecular marker expression in murine JoMa cells}

The murine cell line JoMa1 and the clonally derived subline JoMa1.3 were established and described as immortalized neural crest stem cell lines by Maurer et al. (2007).

To monitor the initial situation both JoMa cell lines were tested for the expression pattern of different molecular marker genes by RT-PCR analysis on cDNA obtained from total RNA of JoMa1 and JoMa1.3 cells (Figure 4 A-C). Whole mouse embryo (E10.5) cDNA was used as positive control for Sox10, Ngfr, Snai2, Pax3, Snai1, Twist1, Tyr and Col2a1. Mouse brain cDNA was used as positive control for Nefh and Gfap, whereas mouse uterus cDNA was used as positive control for $\mathrm{Cnn} 1$ and $A c t g 2 . \mathrm{H}_{2} \mathrm{O}$ was used as negative control, respectively. All analyzed NCC markers were expressed in both JoMa cell lines in exception of Sox10. The neuronal marker Nefh could be detected with a weak signal only in JoMa1 cells, whereas the analyzed glia marker Gfap and the smooth muscle marker Cnn1 were not detected in JoMa cells. The second tested smooth muscle marker gene Actg2 were weakly expressed in both JoMa cell lines. Tyr, the melanocyte marker, was 
detected with a weak signal only in the JoMa1 cells. An expression of the chondrocyte marker Col2a1 was observed in JoMa1 cells as well as in JoMa1.3 cells. Earlier and later cell passages were investigated in the same way with similar results (data not shown).

The data obtained by the RT-PCR analysis demonstrate based on the tested marker genes that JoMa1 and JoMa1.3 cells are undifferentiated NCCs.

A

NCC markers

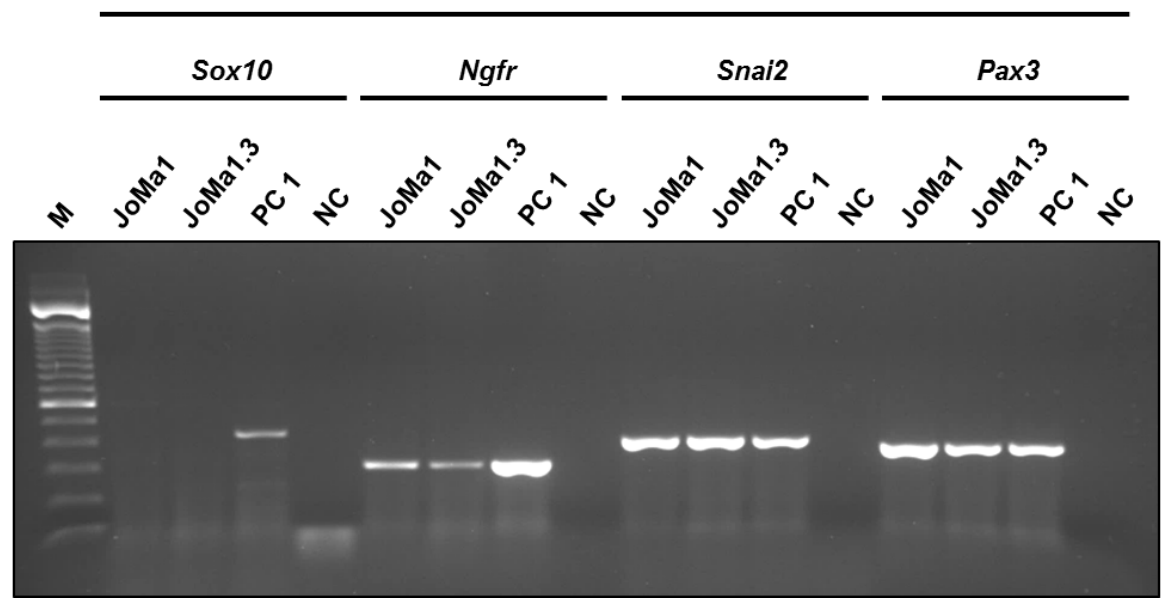

B








Figure 4: Expression analysis of NCC, neuronal, glia, smooth muscle, melanocyte and chondrocyte marker genes in JoMa1 cells and JoMa1.3 cells. RT-PCR analysis performed on cDNA obtained from total RNA of JoMa1 cells and JoMa1.3 cells. The results proved the expression of the NCC markers Ngfr, Snai2, Pax3, Snai1 and Twist1, the neuronal marker Nefh, the smooth muscle marker Actg2, the melanocyte marker Tyr and the chondrocyte marker Col2a1. (A) Expression analysis of the NCC markers Sox10 (444 bp), Ngfr (310 bp), Snai2 (407 bp) and Pax3 (374 bp). (B) Expression analysis of the NCC markers Snai1 (420 bp) and Twist1 (331 bp), the neuronal marker Nefh (301 bp) and the glia marker Gfap (454 bp). (C) Expression analysis of the smooth muscle markers Cnn1 (340 bp) and Actg2 (458 bp), the melanocyte marker Tyr (216 bp) and the chondrocyte marker Col2a1 (321 bp). M: 100 bp DNA Ladder; PC 1: positive control 1 with whole mouse embryo (E10.5) cDNA, PC 2: positive control 2 with mouse brain cDNA, PC 3: positive control 3 with mouse uterus cDNA; NC: negative control with $\mathrm{H}_{2} \mathrm{O}$.

\subsubsection{Molecular marker expression in murine 09-1 cells}

The murine cell line 09-1 was established by Ishii et al. (2012). A whole-genome expression profiling of 09-1 cells revealed that this cell line has characteristics of both neural crest and stem cells (Ishii et al., 2012).

The 09-1 cell line was characterized by RT-PCR analysis on cDNA obtained from total RNA of 09-1 cells using the same marker genes like with the JoMa1 cells and JoMa1.3 cells to monitor the initial situation also of this cell line (Figure $5 \mathrm{~A}-\mathrm{C}$ ). Whole mouse embryo (E10.5) cDNA was used as positive control for Sox10, Ngfr, Snai2, Pax3, Snai1, Twist1, Tyr and Col2a1. Mouse brain cDNA was used as positive control for Nefh and Gfap, whereas mouse uterus cDNA was used as positive control for Cnn1 and Actg2. 
$\mathrm{H}_{2} \mathrm{O}$ was used as negative control, respectively. O9-1 cells express the tested NCC markers although the expression of Pax3 was weak. As in both JoMa cell lines no expression of Sox10 could be detected. Furthermore, there were no expression of the neuronal marker Nefh, the glia marker Gfap and the melanocyte marker Tyr observed. The smooth muscle marker Cnn1 was weak expressed, whereas the other tested smooth muscle marker Actg2 and the chondrocyte marker Col2a1 were strongly expressed. Earlier and later cell passages were investigated in the same way with similar results (data not shown).

The data obtained by the RT-PCR analysis demonstrate based on the tested marker genes that 09-1 cells are, like the JoMa cells, undifferentiated NCCs.

A

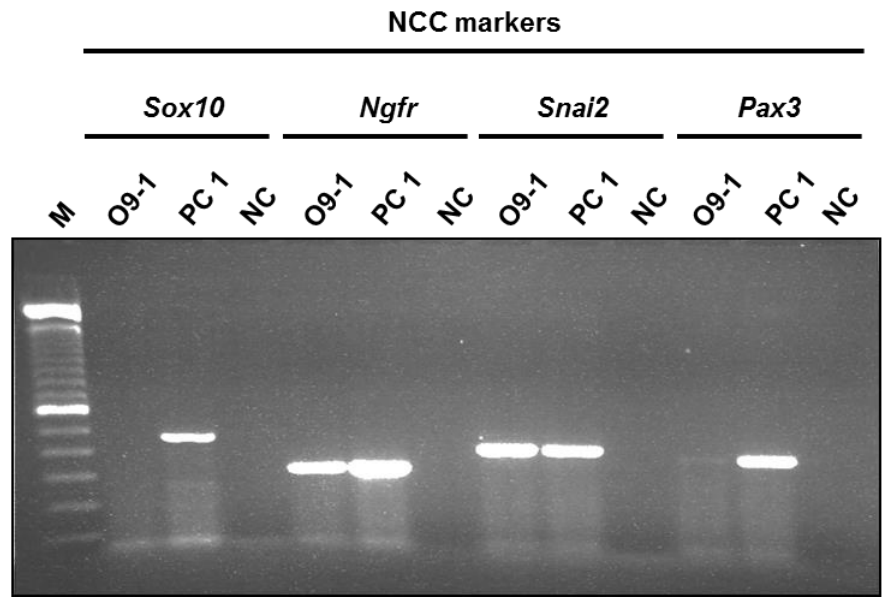

B

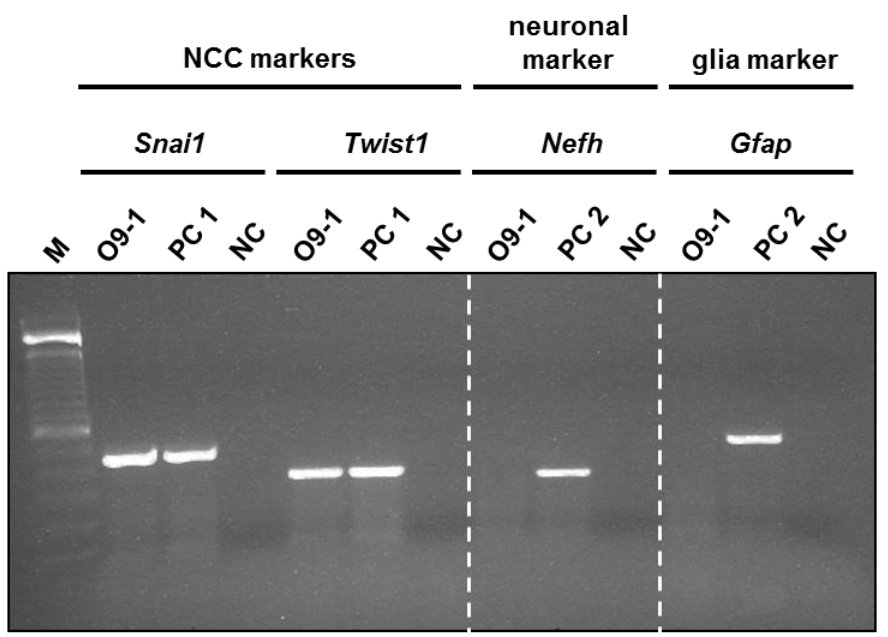




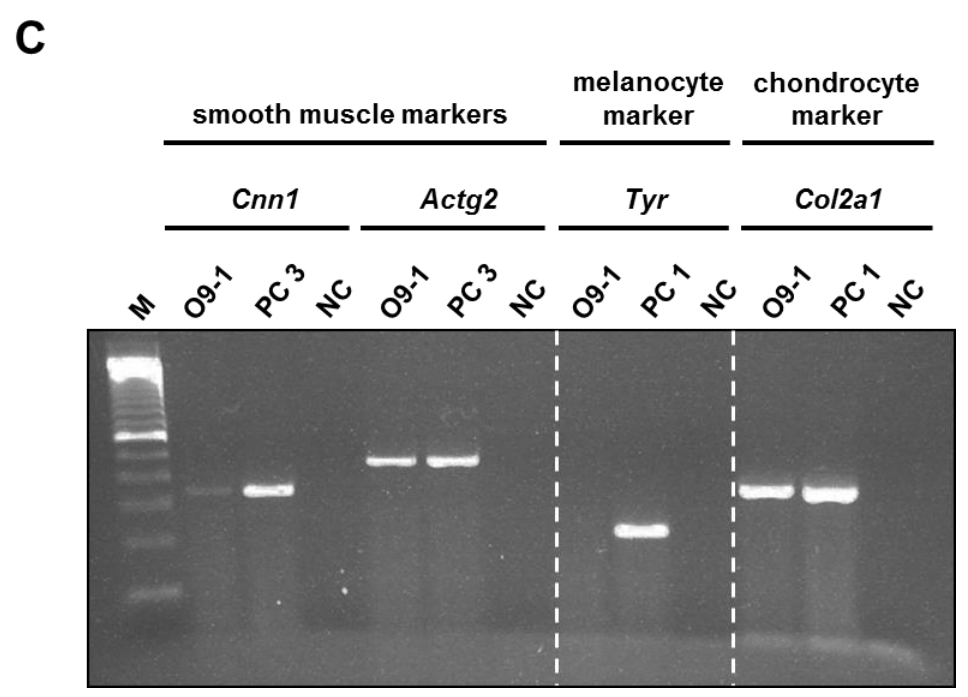

Figure 5: Expression analysis of NCC, neuronal, glia, smooth muscle, melanocyte and chondrocyte marker genes in 09-1 cells. RT-PCR analysis performed on cDNA obtained from total RNA of 09-1 cells. The results revealed the expression of the NCC markers Ngfr, Snai2, Pax3, Snai1 and Twist1, the smooth muscle markers Cnn and Actg2 as well as the chondrocyte marker Col2a1. (A) Expression analysis of the NCC markers Sox10 (444 bp), Ngfr (310 bp), Snai2 (407 bp) and Pax3 (374 bp). (B) Expression analysis of the NCC markers Snai1 (420 bp) and Twist1 (331 bp), the neuronal marker Nefh (301 bp) and the glia marker Gfap (454 bp). (C) Expression analysis of the smooth muscle markers Cnn1 (340 bp) and Actg2 (458 bp), the melanocyte marker Tyr (216 bp) and the chondrocyte marker Col2a1 (321 bp). M: 100 bp DNA Ladder; PC 1: positive control 1 with whole mouse embryo (E10.5) CDNA, PC 2: positive control 2 with mouse brain cDNA, PC 3: positive control 3 with mouse uterus CDNA; NC: negative control with $\mathrm{H}_{2} \mathrm{O}$.

\subsubsection{Chd7 expression in murine JoMa cells and 09-1 cells}

To determine whether JoMa cells and 09-1 cells also express Chd7, the gene mutated in most patients with CHARGE syndrome, RT-PCR analysis on cDNA obtained from total RNA of JoMa cells and 09-1 cells was performed. $\mathrm{H}_{2} \mathrm{O}$ was used as negative control, respectively. As illustrated in Figure $6 \mathrm{~A}$ and B, JoMa1 and JoMa1.3 cells as well as 09-1 cells showed expression of Chd7. 
A

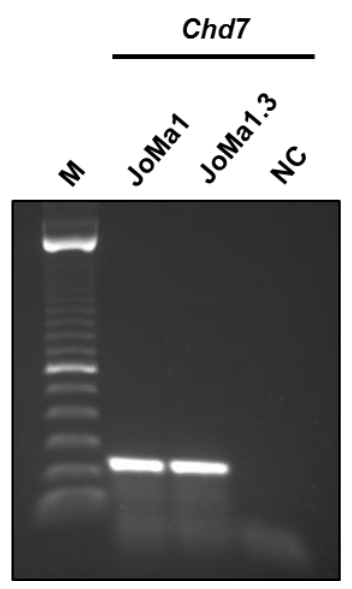

B

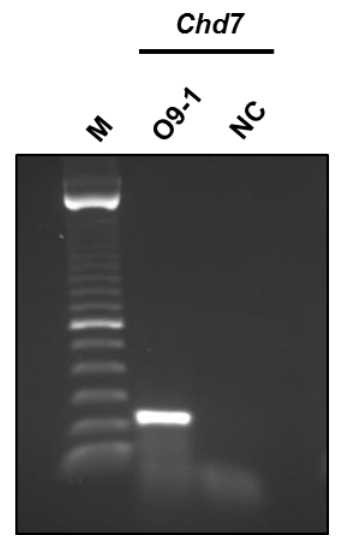

Figure 6: RT-PCR analysis of Chd7 expression in JoMa cells and 09-1 cells. The RT-PCR analysis performed on cDNA obtained from total RNA of (A) JoMa1 and JoMa1.3 cells as well as (B) 09-1 cells proved the expression of Chd7 (238 bp) in both murine NCC lines. M: 100 bp DNA Ladder; NC: negative control with $\mathrm{H}_{2} \mathrm{O}$.

The Chd7 expression was also investigated on protein level. Therefore, western blot analysis with an anti-Chd7 antibody was performed to detect Chd7 using nuclear protein extracts of JoMa cells and 09-1 cells. $30 \mu \mathrm{g}$ of protein was applied per lane. No clear band, but rather a smear could be detected in extracts of JoMa1 and JoMa1.3 cells (Figure $7 \mathrm{~A}$ ). In contrast, a strong $\sim 336 \mathrm{kDa}$ protein band corresponding to the estimated size of Chd7 was received in O9-1 cell extract (Figure 7 B).

A



B

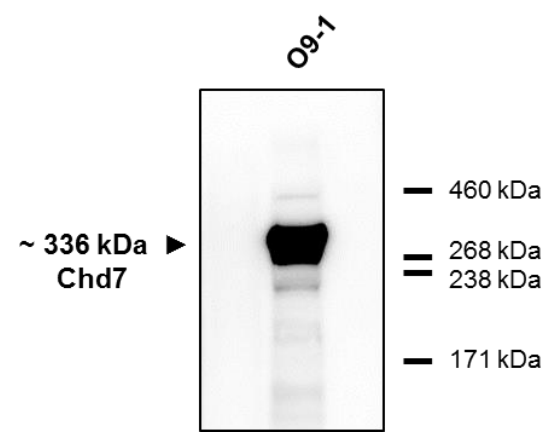

Figure 7: Western blot analysis of Chd7 expression in JoMa cells and 09-1 cells. Western blot analysis using nuclear protein extracts of JoMa1 and JoMa1.3 cells as well as 09-1 cells with an anti-Chd7 antibody to detect Chd7. $30 \mu \mathrm{g}$ of protein was used per lane. (A) A noticeable protein band was received in neither JoMa1 nor JoMa1.3 cell extracts. (B) In the protein extract of 09-1 cells, a protein band corresponding to the estimated size of Chd7 ( 336 kDa) was detected. Molecular weights of proteins and marker bands are indicated in kilo Dalton $(\mathrm{kDa})$. 
The RT-PCR analysis results of the characterized NCC lines revealed that both JoMa cell lines in comparison with the 09-1 cell line differ not much between the tested marker genes and the expression of Chd7. However, the western blot analysis revealed a definite strong Chd7 expression on protein level in 09-1 cells compared to both JoMa cell lines. In addition, 09-1 cells derived from murine cranial NCCs (Ishii et al., 2012), whereas the JoMa cell lines derived from murine trunk NCCs (Maurer et al., 2007). Most of the symptoms seen in CHARGE syndrome patients can be explained by a defect of the induction and migration of cranial NCCs. This is consistent with the observed strong Chd7 expression in the cranial NCC line 09-1. These facts led to the decision to choose the O9-1 cells as a suitable cell line for the investigation of NCCs in vitro.

To confirm the data of the RT-PCR and the western blot analysis concerning the expression of Chd7 in the NCC line 09-1, immunocytochemical staining was performed. Representative images of 09-1 cells are shown in Figure 8. The cell nucleus was stained with DAPI, whereas the cytoskeleton was stained for $\alpha$-Tubulin using an anti- $\alpha$-Tubulin antibody. To detect the target protein Chd7, an anti-Chd7 antibody was used (Figure $8 \mathrm{C}$ ). With secondary antibodies conjugated with either Alexa488 ( $\alpha$-Tubulin) or Cy3 (Chd7), the localization of the proteins were visualized. Cells were incubated without first antibody as negative control. The overlay image revealed that Chd7 was localized specifically in the nucleus (Figure $8 \mathrm{D}$ ).

In summary, the results obtained by western blot analysis and immunocytochemical staining indicate an intense expression of $C h d 7$ in 09-1 cells. 



Figure 8: Chd7 immunocytochemical staining of 09-1 cells. Representative images of 09-1 cells identified the nuclear localization of Chd7. Cell DNA in nucleus was stained with DAPI (blue, A and E) and cytoskeleton was stained with an anti-a-Tubulin antibody (green, B). Localization of Chd7 was analyzed by an anti-Chd7 antibody (red, C). Incubation without first antibody was used as negative control (F and $\mathbf{G})$. Overlay images are shown in the last column (D and H). Alexa488 (B and F) and Cy3 ( $C$ and $G$ ) conjugated secondary antibodies were used for visualization. Representative scale bars are indicated in the overlay images. Scale bars: D: $20 \mu \mathrm{m}$; $\mathrm{H}: 10 \mu \mathrm{m}$.

\subsection{9-1 cells express semaphorin receptors and class 3 semaphorins}

NCCs are known to express semaphorin receptors namely plexins and neuropilins and they are guided by semaphorins during embryogenesis. In order to verify the expression of semaphorin receptors in the NCC line O9-1, RT-PCR analysis was performed on cDNA obtained from total RNA of O9-1 cells. $\mathrm{H}_{2} \mathrm{O}$ was used as negative control, respectively. The results proved the expression of Plxna1 (plexin A1), Nrp1 (neuropilin 1) and Nrp2 (neuropilin 2) (Figure 9). 


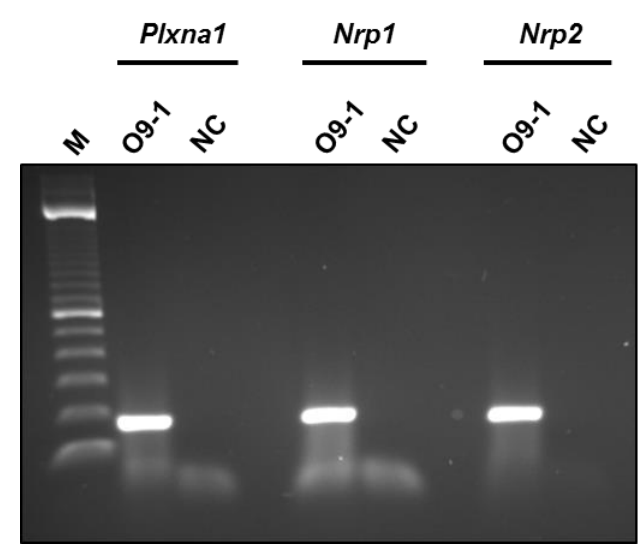

Figure 9: Expression analysis of semaphorin receptors in 09-1 cells. The RT-PCR analysis performed on cDNA obtained from total RNA of 09-1 cells confirmed the expression of Plxna1 (170 bp), Nrp1 (195 bp) and Nrp2 (202 bp). M: 100 bp DNA Ladder; NC: negative control with $\mathrm{H}_{2} \mathrm{O}$.

So far, it is not known that NCCs express semaphorins itself. Nevertheless, RT-PCR analysis on cDNA obtained from total RNA of 09-1 cells revealed the expression of class 3 semaphorins (Sema3a, 3c, 3d and 3e) in NCCs for the first time (Figure 10), suggesting an additional function of these group of semaphorins. $\mathrm{H}_{2} \mathrm{O}$ was used as negative control, respectively.

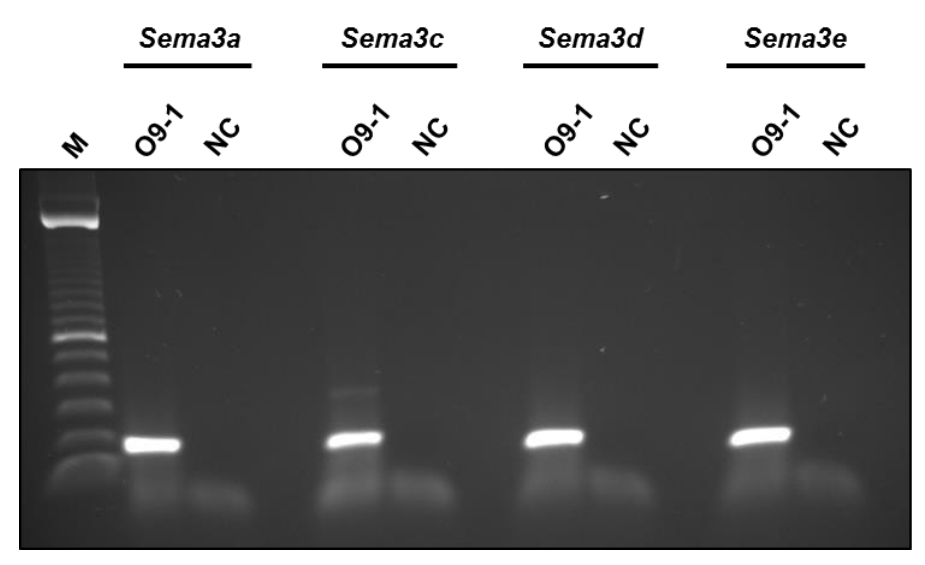

Figure 10: Expression analysis of class 3 semaphorins in 09-1 cells. RT-PCR analysis performed on cDNA obtained from total RNA of 09-1 cells revealed the expression of Sema3a (186 bp), Sema3c (207 bp), Sema3d (186 bp) and Sema3e (183 bp). M: 100 bp DNA Ladder; NC: negative control with $\mathrm{H}_{2} \mathrm{O}$. 
To confirm the data of the RT-PCR analysis concerning the expression of Sema3a in 09-1 cells, immunocytochemical staining was used. Representative images of 09-1 cells are shown in Figure 11. The cell nucleus was stained with DAPI. The cytoskeleton was stained for $\alpha$-Tubulin with an anti- $\alpha$-Tubulin antibody. The target protein Sema3a was detected using an anti-Sema3a antibody (Figure $11 \mathrm{C}$ ). With either Alexa488 ( $\alpha$-Tubulin) or Cy3 (Sema3a) conjugated secondary antibodies, the localization of the proteins were visualized. No staining was detected in negative controls using no primary antibody. The overlay image possessed that Sema3a was localized specifically in the nucleus as well as in the cytoplasm of O9-1 cells (Figure $11 \mathrm{D}$ ).
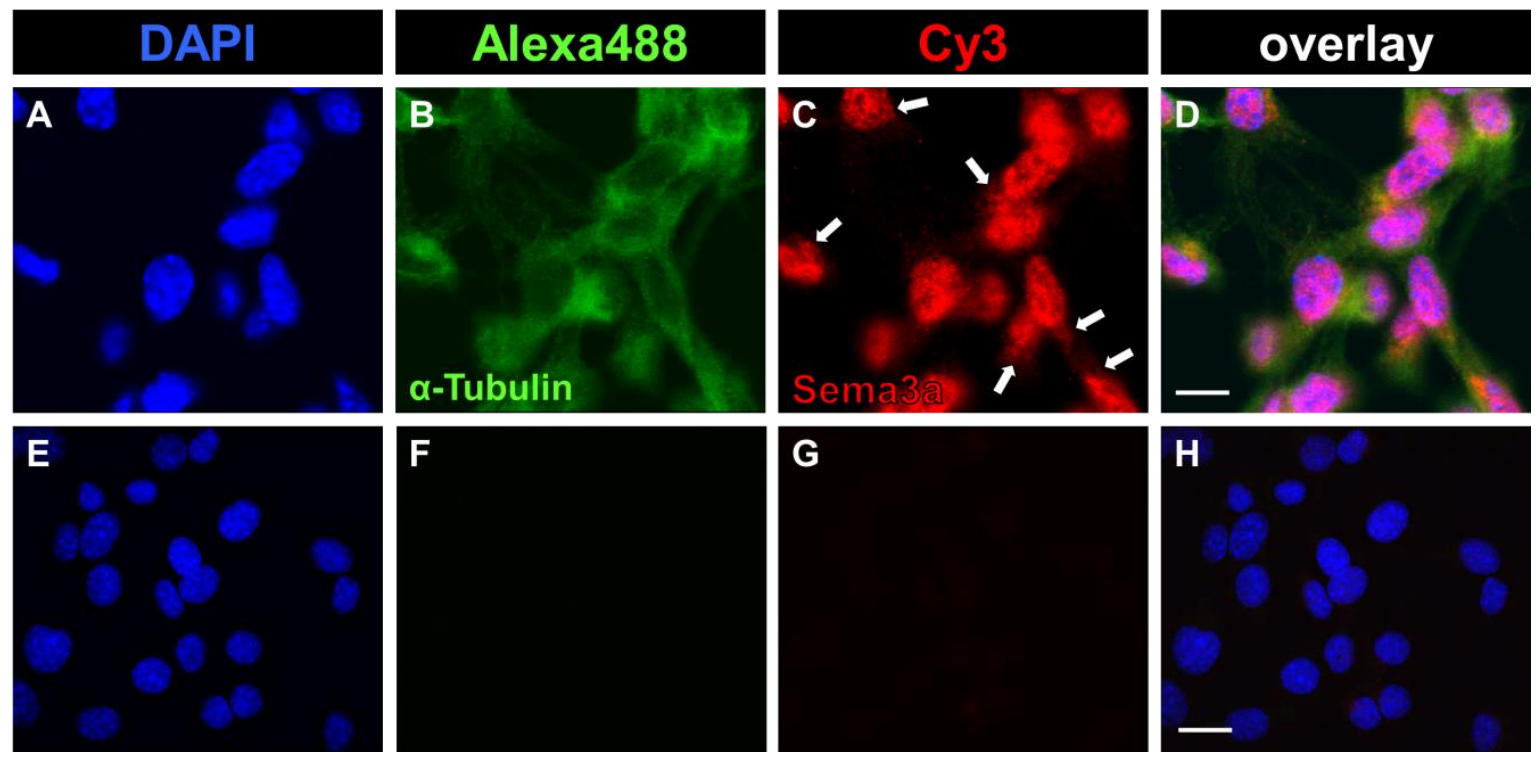

Figure 11: Sema3a immunocytochemical staining of 09-1 cells. Representative images of 09-1 cells revealed cytoplasmic and nuclear localization of Sema3a. Cell DNA in nucleus was stained with DAPI (blue, A and E) and cytoskeleton was stained with an anti- $\alpha$-Tubulin antibody (green, B). Localization of Sema3a was analyzed by an anti-Sema3a antibody (red, C). Red arrows indicate cytoplasm areas, with high accumulation of Sema3a. Incubation without first antibody was used as negative control ( $\mathbf{F}$ and $\mathbf{G}$ ). Overlay images are shown in the last column ( $\mathbf{D}$ and $\mathbf{H}$ ). Alexa488 ( $\mathrm{B}$ and $\mathrm{F}$ ) and Cy3 ( $\mathrm{C}$ and $\mathrm{G}$ ) conjugated secondary antibodies were used for visualization. Representative scale bars are indicated in the overlay images. Scale bars: D: $20 \mu \mathrm{m}$; $\mathrm{H}: 10 \mu \mathrm{m}$. 


\subsection{Sema3a is expressed, processed and secreted by 09-1 cells and exists in monomeric and dimeric isoforms}

Related class 3 semaphorins are described to exist in monomeric, dimeric and processed isoforms (Adams et al., 1997; Christensen et al., 2005). A schematic representation of the full-length Sema3a protein composition with the predicted processing sites and the potential isoforms, resulting from proteolytic processing, is illustrated in Figure 12.

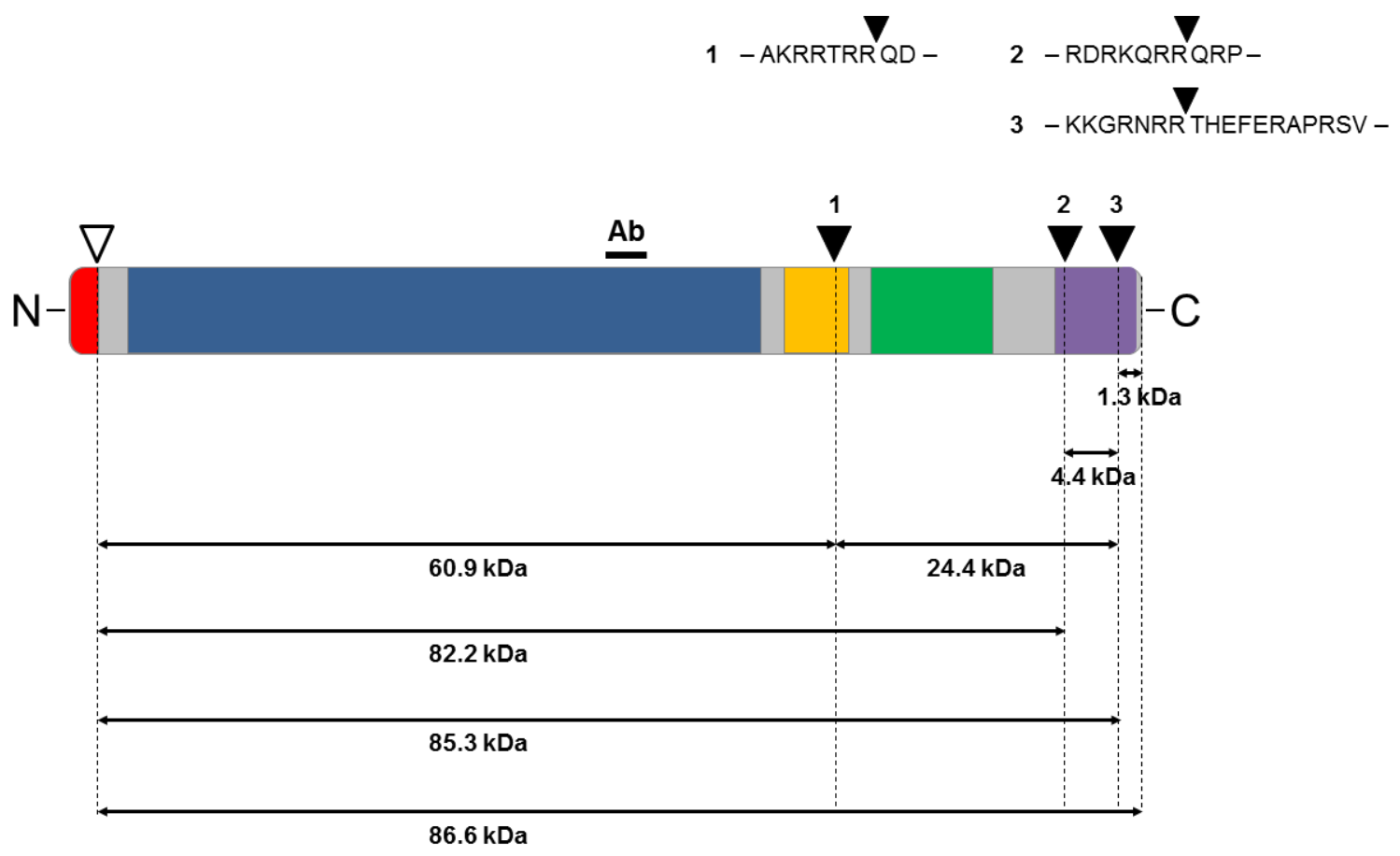

Figure 12: Schematic representation of full-length Sema3a protein. Positions of the signal sequence (red), the Sema (Semaphorin) domain (blue), the PSI (plexin-semaphorin-integrin) domain (yellow), the Ig (immunoglobulin)-like domain (green), the basic domain (purple) and the predicted processing sites 1,2 and 3 (black arrowheads) are indicated. Sema3a sequences containing the processing sites are represented within the figure (top). Processing site after signal sequence (open arrowhead) is permanently used. Theoretical molecular weights of cleavage products are shown in kilo Dalton $(\mathrm{kDa})$. Ab: binding region of the used anti-Sema3a antibody. Adapted from Adams et al. (1997) and Christensen et al. (2005).

After Sema3a expression was identified in 09-1 cells by RT-PCR analysis (chapter 3.2, Figure 10), the expression of Sema3a was investigated on protein level using cytoplasmic and nuclear 09-1 protein extracts. Secreted Sema3a was analyzed using concentrated 
cell culture medium of 09-1 cells. The cytoplasmic and nuclear protein extracts were separately isolated and the medium was concentrated after $24 \mathrm{~h}$ of usage. In order to check monomeric and dimeric Sema3a protein isoforms, the different proteins were treated with the reducing agent DTT (+DTT) or without reducing agent (-DTT). Medium and protein extracts were analyzed by western blot analysis using an anti-Sema3a antibody and $30 \mu \mathrm{g}$ of protein per lane.

The representative results are shown in Figure 13. The monomeric isoforms of $\sim 87 \mathrm{kDa}$ (p87), 85 kDa (p85), 82 kDa (p82) and $\sim 61 \mathrm{kDa}$ (p61) were observed in concentrated medium with reduction, whereas the dimeric isoforms of $\sim 174 \mathrm{kDa}$ (p87:p87) and $\sim 170 \mathrm{kDa}$ (p85:p85) as well as the monomeric isoforms of $\sim 82 \mathrm{kDa}$ (p82) and $\sim 61 \mathrm{kDa}$ (p61) were detected in concentrated medium without reduction. In cytoplasmic extract with reducing agent, the dimeric isoform of $\sim 170 \mathrm{kDa}(\mathrm{p} 85: \mathrm{p} 85)$ and the monomeric isoform of 85 kDa (p85) were observed. The dimeric isoform p85:p85 possibly indicates an incomplete cleavage of the disulfide bond between the dimer although the reducing agent DTT was added. In cytoplasmic extract without reducing agent, the dimeric isoform of $\sim 170 \mathrm{kDa}$ (p85:p85) was received. The monomeric isoform of $\sim 85 \mathrm{kDa}$ (p85) was observed in nuclear extract with reduction, whereas the dimeric isoform of $\sim 170 \mathrm{kDa}$ (p85:p85) was received without reduction. In nuclear extract, with and without addition of reducing agent, potentially unspecific protein bands due to unspecific binding of the used antibody were detected. To proof this assumption, 09-1 cells with a full-lengths Sema3a plasmid containing a HA-tag between the signal sequence and the semaphorin domain should be performed. By detection the tag using an anti-HA antibody, the same protein band pattern without the potentially unspecific bands should be received.

Detection of $\mathrm{a}$-Tubulin ( $50 \mathrm{kDa}$ protein band) served as loading control for the cytoplasmic extracts. However, the detection of $\alpha$-Tubulin in the nuclear extracts revealed the additional existence of cytoplasmic proteins. This fact can be possibly explained by the used protein extraction method. The nuclear protein Baf47 ( 47 kDa protein band) was solely received in the nuclear extracts.

Collectively, the investigation of Sema3a in 09-1 cells on protein level revealed that the expressed full-length Sema3a protein undergoes proteolytic processing to shorter isoforms. Moreover, the existence of monomeric and dimeric isoforms as well as the secretion of Sema3a was demonstrated. The obtained results are summarized in Table 1 and Figure 14. 


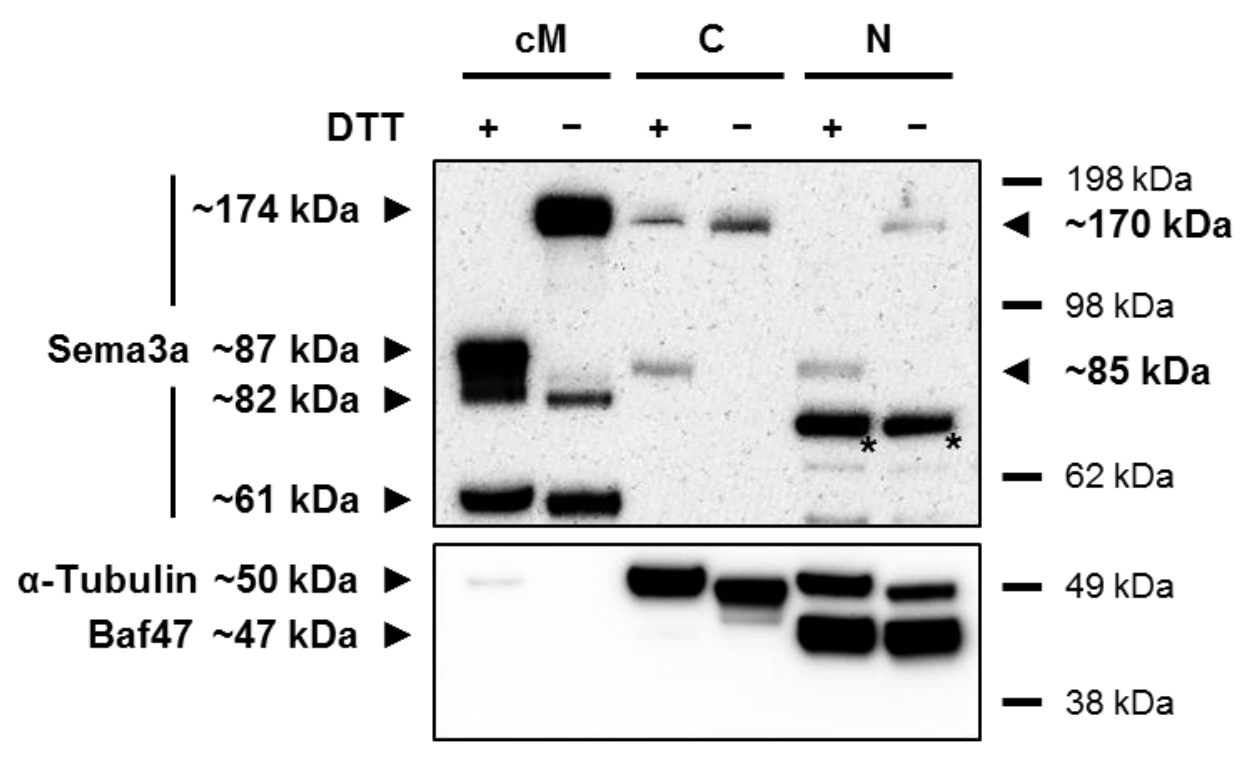

Figure 13: Isoforms of Sema3a resulting from proteolytic processing and dimerization. Cell culture medium was concentrated (cM) after $24 \mathrm{~h}$ of usage. Cytoplasmic (C) and nuclear (N) protein extracts of 09-1 cells were separately isolated. $30 \mu \mathrm{g}$ of protein treated with the reducing agent DTT (+DTT) or without reducing agent (-DTT) was used per lane. The Sema3a isoforms were analyzed by western blot analysis using an anti-Sema3a antibody. In concentrated medium with reducing agent the monomeric isoforms of $\sim 87 \mathrm{kDa}(\mathrm{p} 87), \sim 85 \mathrm{kDa}(\mathrm{p} 85), \sim 82 \mathrm{kDa}(\mathrm{p} 82)$ and $\sim 61 \mathrm{kDa}$ (p61) were detected, whereas in concentrated medium without reducing agent the dimeric isoforms of $\sim 174 \mathrm{kDa}$ (p87:p87) and $\sim 170 \mathrm{kDa}$ (p85:p85) and the monomeric isoforms of $\sim 82 \mathrm{kDa}$ (p82) and $\sim 61 \mathrm{kDa}$ (p61) were observed. In cytoplasmic extract with reduction, the dimeric isoform of $\sim 170 \mathrm{kDa}$ (p85:p85) and the monomeric isoform of $\sim 85 \mathrm{kDa}$ (p85) were received. The dimeric isoform p85:p85 possibly indicates an incomplete cleavage of the disulfide bond between the two p85 monomeric isoforms despite DTT addition. Without reduction, solely the dimeric isoform of $\sim 170 \mathrm{kDa}$ (p85:p85) was observed. In the nuclear extract with reducing agent, the monomeric isoform of $\sim 85 \mathrm{kDa}$ (p85) was received, whereas without reducing agent the dimeric isoform of $\sim 170 \mathrm{kDa}$ (p85:p85) was observed. The detection of $\alpha$-Tubulin ( $50 \mathrm{kDa}$ protein band) served as loading control for the cytoplasmic extracts, revealing the additional existence of cytoplasmic proteins also in the nuclear extracts which can be possible traced back to the used protein extraction method. The nuclear protein Baf47 ( $47 \mathrm{kDa}$ protein band) was exclusively detected in the nuclear extracts. Stars $(*)$ illustrate potentially unspecific protein bands due to unspecific binding of the anti-Sema3a antibody. Molecular weights of proteins and marker bands are indicated in kilo Dalton $(\mathrm{kDa})$. 
Table 1: Sema3a isoforms detected in concentrated medium as well as cytoplasmic and nuclear protein extracts of 09-1 cells. Medium and protein extracts were treated with the reducing agent DTT (+DTT) and without reducing agent (-DTT) and analyzed by western blot analysis using an anti-Sema3a antibody. Approximate molecular weights of the isoforms are given in kilo Dalton $(\mathrm{kDa})$.

\begin{tabular}{c|c|c|c|c|c}
\hline \multicolumn{2}{c|}{$\begin{array}{c}\text { concentrated medium } \\
\text { +DTT }\end{array}$} & \multicolumn{2}{c|}{ cytoplasmic extract } & \multicolumn{2}{c}{ nuclear extract } \\
\hline $87 \mathrm{kDa}$ & $174 \mathrm{kDa}$ & $170 \mathrm{kDa}$ & $170 \mathrm{kDa}$ & $85 \mathrm{kDa}$ & $170 \mathrm{kDa}$ \\
$85 \mathrm{kDa}$ & $170 \mathrm{kDa}$ & $85 \mathrm{kDa}$ & & & \\
$82 \mathrm{kDa}$ & $82 \mathrm{kDa}$ & & & & \\
$61 \mathrm{kDa}$ & $61 \mathrm{kDa}$ & & & & \\
\hline
\end{tabular}

A

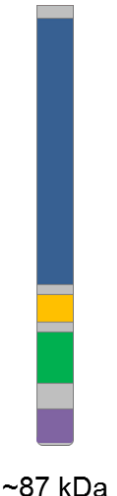

p87

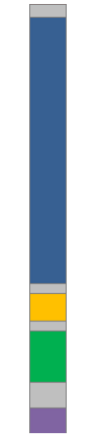

$\sim 85 \mathrm{kDa}$

p85

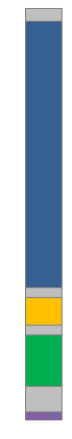

$\sim 82 \mathrm{kDa}$

p82

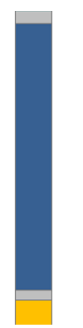

$\sim 61 \mathrm{kDa}$

p61

B

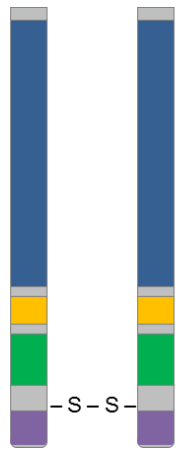

$\sim 174 \mathrm{kDa}$

p87:p87

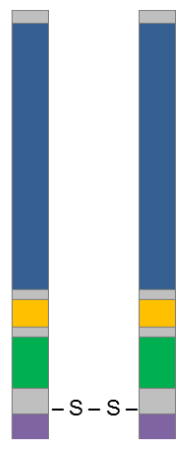

$\sim 170 \mathrm{kDa}$

p85:p85

Figure 14: Schematic representation of proteolytic processed and dimerized Sema3a isoforms identified in concentrated medium as well as cytoplasmic and nuclear protein extracts of 09-1 cells. Isoforms resulting from combined proteolytic processing and dimerization of Sema3a full length protein are shown, which were detectable with the used anti-Sema3a antibody by western blot analysis. (A) Four monomeric isoforms were observed: $\sim 87 \mathrm{kDa}$ (p87), $\sim 85 \mathrm{kDa}$ (p85), $\sim 82 \mathrm{kDa}$ (p82) and $\sim 61 \mathrm{kDa}$ (p61). (B) Two dimeric isoforms were detected: the dimeric isoform of $\sim 174 \mathrm{kDa}$ (p87:p87) consist of two $\sim 87 \mathrm{kDa}$ (p87) monomeric isoforms, whereas the dimeric isoform of $\sim 170 \mathrm{kDa}$ (p85:p85) consist of two $\sim 85 \mathrm{kDa}$ (p85) monomeric isoforms. Disulfide bonds between the dimeric isoforms are indicated with $-\mathrm{S}-\mathrm{S}-$. Approximate molecular weights of the isoforms are displayed in kilo Dalton $(\mathrm{kDa})$ and in short version ( $\mathrm{p}$ ) (bottom). Adapted from Adams et al. (1997) and Christensen et al. (2005). 


\subsection{Downregulation of Chd7 has influence on the expression level of Sema3a in 09-1 cells}

In order to determine whether the downregulation of Chd7 has an influence on the expression level of Sema3a in NCCs, murine 09-1 cells, which have a high basal Chd7 expression level (chapter 3.1.3), were transfected with one of three different short interfering RNAs (siRNA 1, 2, 3) against Chd7. Total RNA lysates were isolated from O9-1 cells $48 \mathrm{~h}$ after siRNA transfection and reverse transcribed into cDNA. Afterwards, the efficiency of the siRNAs was tested by qRT-PCR analysis. All three Chd7-specific siRNAs proved to be effective as seen by a reduced Chd7 expression compared with control siRNA transfected cells (Figure 15). With siRNA 1 or siRNA 3 transfected 09-1 cells had a significantly reduced relative Chd7 expression between $65-70 \%$.

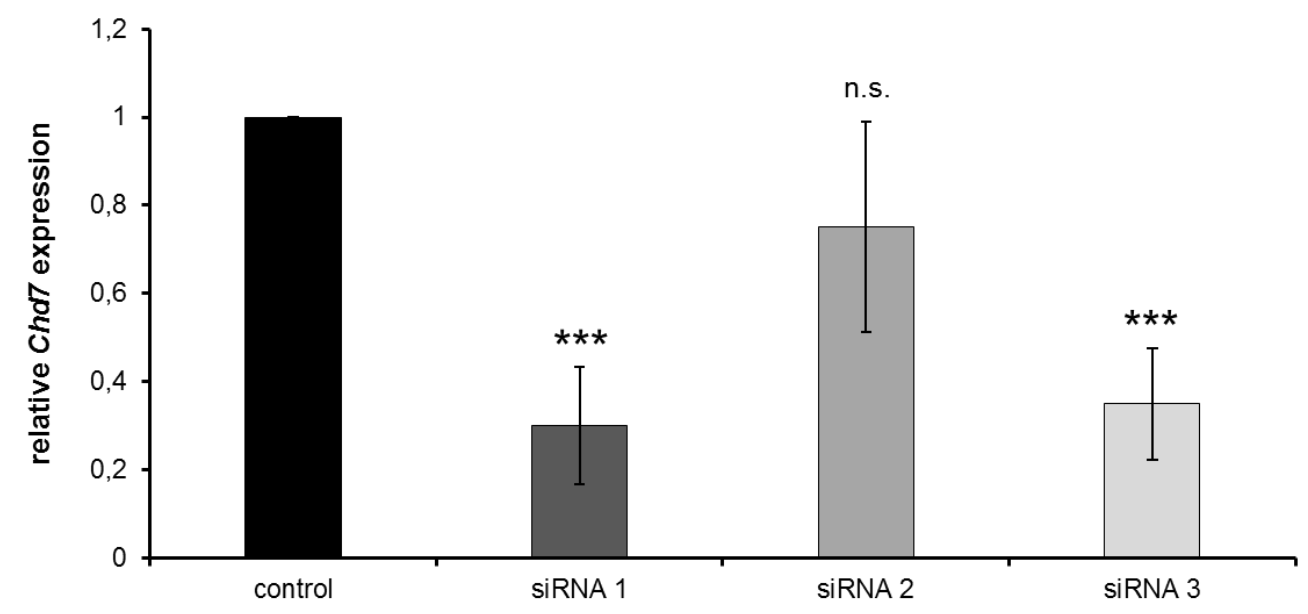

Figure 15: Analysis of the Chd7 expression in 09-1 cells after Chd7 downregulation. 09-1 cells were transfected with one of three different Chd7-specific siRNAs (siRNA 1, 2, 3). Total RNA lysates of transfected cells were isolated $48 \mathrm{~h}$ after siRNA transfection and reverse transcribed into cDNA which was subjected to qRT-PCR analysis. The transfection of Chd7specific siRNAs induced a reduction of the Chd7 expression compared with control siRNA transfected cells, demonstrating the effectiveness of the siRNAs. With siRNA 1 or siRNA 3 transfected cells had a significantly decreased relative Chd7 expression between $65-70 \%$. Data are presented as mean \pm standard of qRT-PCR analysis performed in triplicate. ${ }^{* * *} p \leq 0.001$, n.s.: not significant (student's $t$ test). 
After demonstrating the effectiveness especially of the Chd7-specific siRNA 1 and siRNA 3, the influence on the Sema3a expression was investigated. Therefore, total RNA lysates of 09-1 cells transfected with siRNA 1 or siRNA 3 were isolated $48 \mathrm{~h}$ after transfection. Isolated RNA was reverse transcribed into cDNA and used for qRT-PCR analysis. With Chd7-specific siRNA 1 or siRNA 3 transfected cells had a significantly decreased relative Sema3a expression of the approximately $30 \%$ in comparison with control siRNA transfected cells (Figure 16).

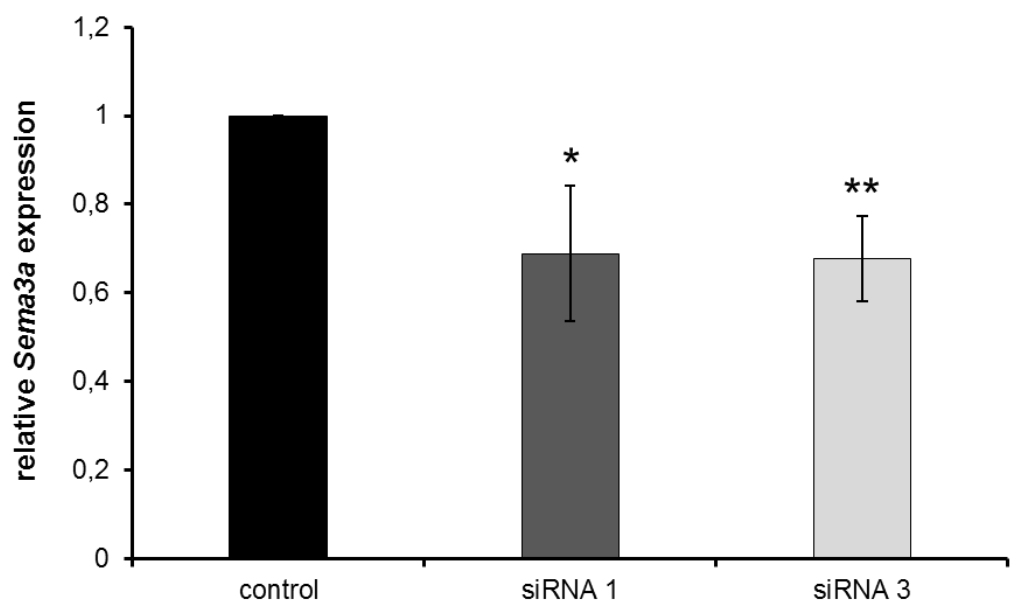

Figure 16: Analysis of the Sema3a expression in 09-1 cells after Chd7 downregulation. Total RNA lysates of 09-1 cells transfected with one of two different Chd7-specific siRNAs (siRNA 1,3 ) were isolated $48 \mathrm{~h}$ after transfection. Into cDNA reverse transcribed RNA was subjected to qRT-PCR analysis to check the Sema3a expression after Chd7 downregulation. Both single transfected Chd7-specific siRNAs induced a significant reduction of the relative Sema3a expression of approximately $30 \%$ compared with control siRNA transfected cells. Data are presented as mean \pm standard of qRT-PCR analysis performed in triplicate. ${ }^{*} p \leq 0.05,{ }^{* *} p \leq 0.01$ (student's $t$ test).

\subsection{Chd7 co-immunoprecipitates with Sema3a in 09-1 cells}

In order to analyze the existence of $\mathrm{Chd7}$ and Sema3a in the same nuclear protein complex in NCCs, co-immunoprecipitation (Co-IP) experiments using protein extract of 09-1 cells were performed. Nuclear extract was incubated with an anti-Chd7 antibody to precipitate Chd7. By western blot analysis using an anti-Sema3a antibody, a $\sim 85 \mathrm{kDa}$ protein band corresponding to the estimated size of the monomeric p85-Sema3a isoform was detected (Figure $17 \mathrm{~A}$ ). This band is probable more intense than in the input control 
due to the high amount of applied protein in immunoprecipitation and a strong binding of the anti-Chd7 antibody. To verify the results in a reciprocal experiment, nuclear extract was incubated with an anti-Sema3a antibody to precipitate Sema3a. A 336 kDa protein band corresponding to the estimated size of Chd7 was detected by western blot analysis using an anti-Chd7 antibody (Figure 17 B). Nuclear extract without antibody incubation served as negative control to prove that Sema3a (Figure 17 A) and Chd7 (Figure 17 B) are not capable to bind unspecific to the magnetic beads used for the Co-IP experiments. $30 \mu \mathrm{g}$ of nuclear protein per lane was used as input control to demonstrate that Sema3a (Figure $17 \mathrm{~A}$ ) and Chd7 (Figure $17 \mathrm{~B}$ ) are expressed in the nucleus of 09-1 cells.

In conclusion, the data obtained by the Co-IP experiments demonstrate an interaction of Chd7 with Sema3a in 09-1 cells.

A

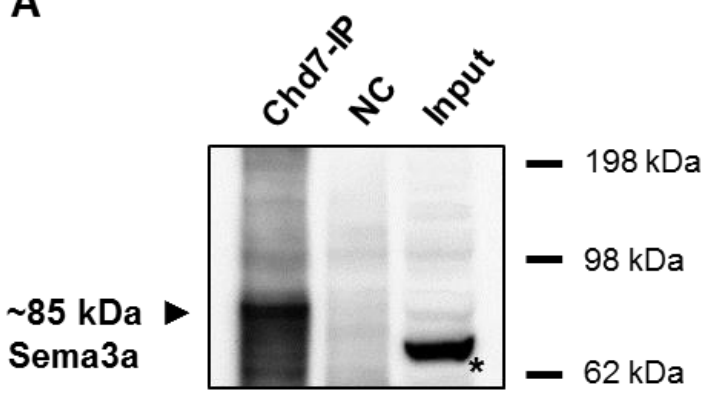

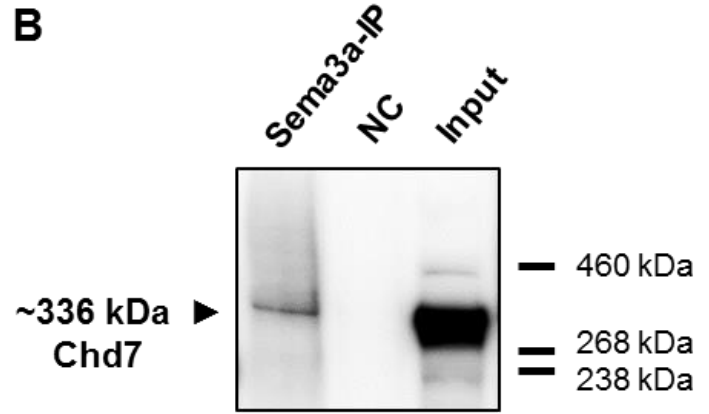

Figure 17: Co-immunoprecipitation of Chd7 with Sema3a in 09-1 cells. The Co-IP experiments were performed with nuclear protein extract of 09-1 cells. (A) Chd7 was precipitated with an anti-Chd7 antibody. A $\sim 85 \mathrm{kDa}$ protein band corresponding to the estimated size of the monomeric p85-Sema3a isoform was detected by western blot analysis using an anti-Sema3a antibody. This band is probable more intense than in the input control due to the high amount of applied protein in immunoprecipitation and a strong binding of the anti-Chd7 antibody. (B) The reciprocal experiment was performed to verify the results. Sema3a was precipitated with an anti-Sema3a antibody. By western blot analysis using an anti-Chd7 antibody, a $~ 336 \mathrm{kDa}$ protein band corresponding to the estimated size of Chd7 was detected. Nuclear extract without antibody incubation was used as negative control (NC) to exclude unspecific binding of Sema3a (A) or Chd7 (B) to the magnetic beads used for the Co-IP experiments. $30 \mu \mathrm{g}$ of nuclear protein per lane was used as input control to demonstrate the expression of Sema3a (A) and Chd7 (B) in the nucleus of 09-1 cells. Star $(*)$ illustrate potentially unspecific protein band due to unspecific binding of the used anti-Sema3a antibody. Molecular weights of proteins and marker bands are indicated in kilo Dalton $(\mathrm{kDa})$. 


\subsection{Detection of the non-synonymous SEMA3A variant c.2002A>G (p.1668V) in a CHD7-positive CHARGE syndrome patient}

CHARGE syndrome is described as a phenotypically heterogeneous disorder. For this reason, it might be possible that there are other genes in addition to $\mathrm{CHD7}$ which might act as modifiers leading to a mild or a severe phenotype when these genes are mutated. One of these candidate genes could be $S E M A 3 A$, known to contribute to the phenotype of the CHARGE-related disorder Kallmann syndrome. Therefore, 5 CHARGE patients with a mild phenotype and 10 CHARGE patients with a severe phenotype, but all carrying pathogenic mutations in $C H D 7$, were screened for additional mutations in SEMA3A (MIM 603961). All 17 coding exons and flanking intronic sequences of SEMA3A (NM_006080.2) were analyzed by amplifying these regions by PCR using genomic DNA and screened for mutations by sequencing analysis. In one patient with a severe phenotype, the non-synonymous variant c.2002A $>G$ (p.l668V) was found (Figure 18) which is located in the Ig-like domain of SEMA3A (Figure 19). While the in silico programs PolyPhen-2 and SIFT predicted that this new variant is tolerated, the program MutationTaster predicted it as disease causing. Moreover, the alteration is not listed in the database of the Exome Aggregation Consortium (ExAC). Hanchate et al. (2012) characterized two other SEMA3A mutations: the variant c.196C>T (p.R66W) should lead to an impaired SEMA3A secretion, whereas the variant c.2062A>G (p.T688A) should reduce the signal activity of SEMA3A. To clarify the new variant $p .1668 \mathrm{~V}$, functional analysis on human embryonic kidney 293 cells (HEK293 cells) were performed. The wild type SEMA3A and the SEMA3A variant p.R66W were used as referees. 


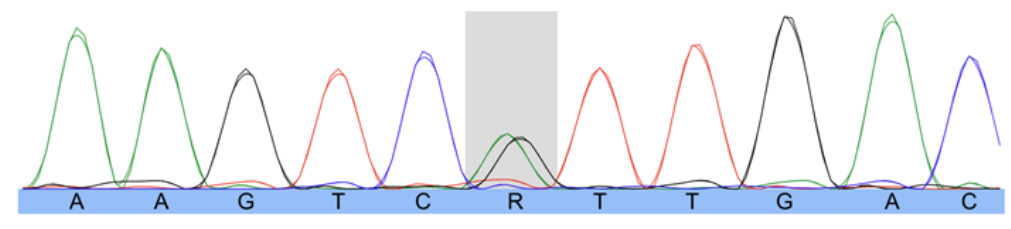

forward sequence

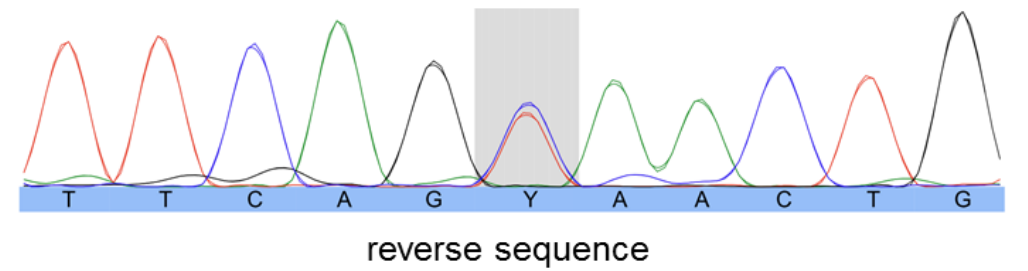

Figure 18: SEMA3A mutation found in a CHD7-positive CHARGE syndrome patient. All 17 coding exons and flanking intronic sequences of the SEMA3A gene were sequenced in 5 CHARGE patients with a mild phenotype and 10 CHARGE patients with a severe phenotype, but all carrying pathogenic mutations in the $C H D 7$ gene. One non-synonymous variant was identified in a patient with a severe phenotype: c. $2002 \mathrm{~A}>\mathrm{G}$ (p.1668V). The mutation is indicated by gray background in the forward and the reverse sequence, respectively.

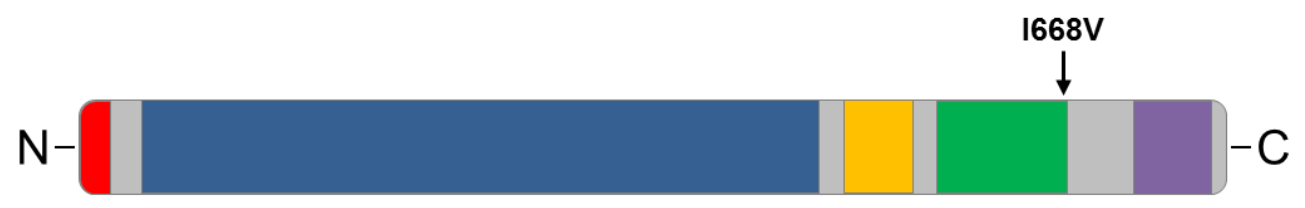

Figure 19: Schematic representation of full length SEMA3A protein with identified mutation. The non-synonymous variant c.2002A $>\mathrm{G}$ (p.1668V), which was identified in a CHD7-positive CHARGE syndrome patient, is located in the Ig-like domain (green) of SEMA3A.

\subsection{Generation of SEMA3A plasmids}

The plasmid SEMA3A-WT-pcDNA3.1/myc-His B (NP_006071.1, kindly provided by my research group), which contains the human SEMA3A wild type (WT) sequence, was used to generate pcDNA3.1/myc-His B plasmids carrying the c.196C>T (p.R66W) and the c. $2002 A>G$ (p.1668V) mutation, respectively. The plasmid with the variant c.196C>T (p.R66W) was used as referee, because this mutation was characterized by Hanchate 
et al. (2012) leading to an impaired SEMA3A secretion. The mutations were generated using the QuikChange II XL Site-Directed Mutagenesis Kit (Agilent Technologies) (chapter 2.2.1.4.4). The generated plasmids SEMA3A-R66W-pcDNA3.1/myc-His B and SEMA3A-1668V-pcDNA3.1/myc-His B were validated for the correct nucleotide sequence and reading frame by sequencing analysis.

Afterwards, the SEMA3A WT sequence and the two SEMA3A mutation sequences were separately cloned into the pCMV-HA vector (Invitrogen), containing a hemagglutinin (HA) epitope tag, as well as into the pGADT7 vector (Clontech) using the In-Fusion ${ }^{\circledR}$ HD Cloning Kit (Clontech) (chapter 2.2.1.4.2). Sequencing analysis was used to validate the correct nucleotide sequence and reading frame of the generated plasmids. The analysis revealed the existence of the HA-tag in front of the SEMA3A signal sequence in the generated plasmids.

\subsection{SEMA3A variants have no effect on the expression, processing and secretion as well as dimerization of SEMA3A}

The effect of the SEMA3A variant p.1668V, which was detected in a CHD7-positive CHARGE syndrome patient (chapter 3.6), was analyzed concerning expression, processing and dimerization as well as secretion of SEMA3A. As referees, SEMA3A WT and the SEMA3A variant p.R66W were used.

Therefore, transient overexpression of SEMA3A WT and SEMA3A variants was performed with the generated SEMA3A-pCMV-HA plasmids (chapter 3.7) using HEK293 cells. This cell line was chosen to test the plasmids in a human cell system by western blot analysis.

HEK293 cells were single transfected with SEMA3A-pCMV-HA plasmids containing the human sequence of SEMA3A WT or of the SEMA3A variants c.196C >T (p.R66W) and c. $2002 A>G$ (p.1668V) to initially analyze the expression of the overexpressed plasmids and if the overexpressed SEMA3A undergoes proteolytic processing. HEK293 cells without plasmid treatment (untransfected) were used to investigate the SEMA3A expression level. Cytoplasmic and nuclear protein extracts were separately isolated $24 \mathrm{~h}$ after transfection. Western blot analysis was performed using $30 \mu \mathrm{g}$ of protein per lane. Endogenous and overexpressed SEMA3A was detected with an anti-SEMA3A antibody 
(Figure $20 \mathrm{~A}$ ), while an anti-HA antibody was used for the detection of the plasmid containing HA-tag (Figure $20 \mathrm{~B}$ ). In cytoplasmic and nuclear protein extracts of untransfected HEK293 cells only two potentially unspecific protein bands due to unspecific binding of the used anti-SEMA3A antibody were detected, demonstrating that HEK293 cells express almost undetectable levels of SEMA3A (Figure 20 A). In cytoplasmic and nuclear protein extracts of transfected HEK293 cells the monomeric SEMA3A isoforms of $\sim 87 \mathrm{kDa}$ (p87) and $\sim 85 \mathrm{kDa}$ (p85) were detected (Figure $20 \mathrm{~A}$ ), suggesting proteolytic processing of the overexpressed SEMA3A. Moreover, the results demonstrate that the overexpressed SEMA3A WT plasmid in comparison with the overexpressed SEMA3A variant plasmids show the same expression pattern in the nucleus and cytoplasm of HEK293 cells.

Using an anti-HA antibody, no protein bands were detected neither in the cytoplasmic nor in the nuclear protein extracts except from the positive control, indicating the HA-tag gets lost in HEK293 cells after transfection of the SEMA3A plasmids due to proteolytic cleavage (Figure $20 \mathrm{~B}$ ). For this reason the HA-tag could not be detected with the anti-HA antibody. Nevertheless, due to an almost undetectable SEMA3A expression level in HEK293 cells, the overexpressed SEMA3A was detected in further experiments using an anti-SEMA3A antibody. To proof the assumption concerning the unspecific protein bands, HEK293 cells should be transfected with SEMA3A WT and SEMA3A variant plasmids containing a HA-tag between the signal sequence and the semaphorin domain. Consequently, the potentially unspecific protein bands should not be detected using an anti-HA antibody.

Detection of $\alpha$-Tubulin ( $50 \mathrm{kDa}$ protein band) served as loading control (Figure $20 \mathrm{~B}$ ). Although $\alpha$-Tubulin is expressed in the cytoplasm it was also detected in the nuclear protein extracts which can possibly be traced back on the used protein extraction method. 
A

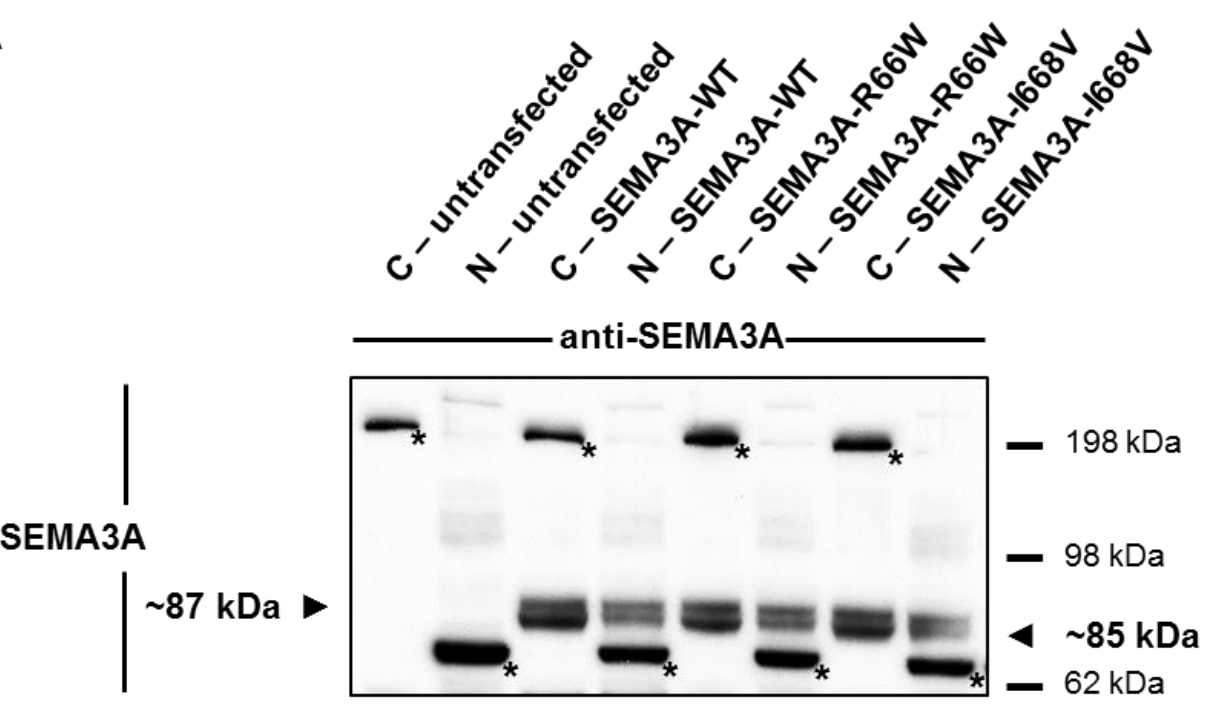

B

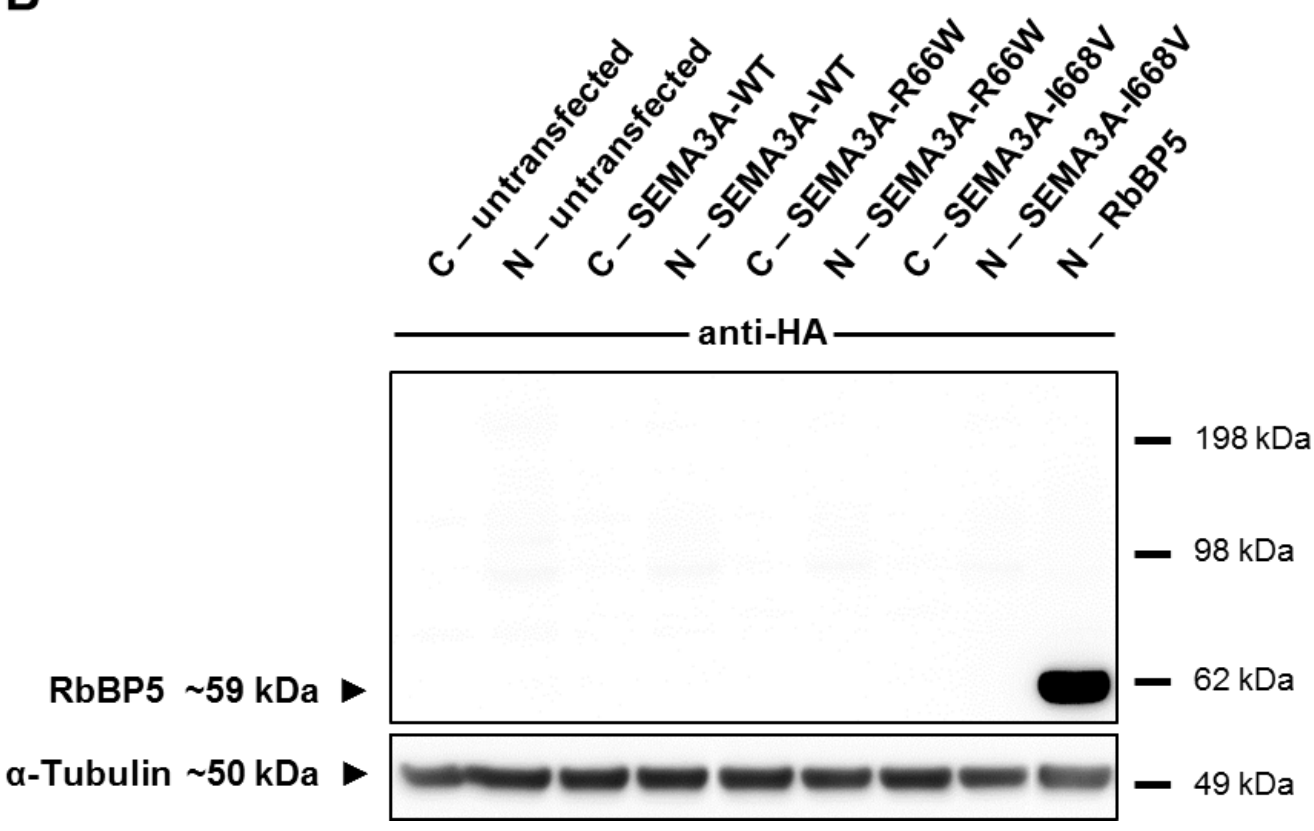

Figure 20: Expression and proteolytic processing of transient overexpressed SEMA3A WT and SEMA3A variants in HEK293 cells. HEK293 cells were single transfected with SEMA3APCMV-HA plasmids containing the human sequence of SEMA3A WT or of the SEMA3A variants c.196C>T (p.R66W) and c.2002A>G (p.1668V). Cytoplasmic $(\mathrm{C})$ and nuclear $(\mathrm{N})$ protein extracts were separately isolated $24 \mathrm{~h}$ after transfection. $30 \mu \mathrm{g}$ of protein was used per lane. (A) Endogenous and overexpressed SEMA3A was detected by western blot analysis using an antiSEMA3A antibody. In untransfected cytoplasmic and nuclear extracts no specific SEMA3A protein bands were detected, demonstrating that HEK293 cells express almost undetectable levels of SEMA3A. In transfected cytoplasmic and nuclear extracts the monomeric SEMA3A isoforms of $\sim 87 \mathrm{kDa}$ (p87) and $\sim 85 \mathrm{kDa}$ (p85) were observed, suggesting proteolytic processing of the 
overexpressed SEMA3A. Nevertheless, no differences in the cytoplasmic and nuclear expression pattern of the overexpressed SEMA3A WT plasmid in comparison with the overexpressed SEMA3A variant plasmids were observed. Stars $(*)$ illustrate potentially unspecific protein bands due to unspecific binding of the used anti-SEMA3A antibody. (B) In the transfected cytoplasmic and nuclear extracts no SEMA3A protein bands were detected with an anti-HA antibody, suggesting the HA-tag loss of the generated SEMA3A plasmids due to proteolytic cleavage. Nuclear protein extract of HEK293 cells transfected with RbBP5-pCMV-HA plasmid ( $\sim 59 \mathrm{kDa}$ protein band) was used as positive control. The detection of $\alpha$-Tubulin ( $50 \mathrm{kDa}$ protein band) served as loading control. Due to the protein extraction method, $\alpha$-Tubulin was detected in the cytoplasmic and the nuclear protein extracts. Molecular weights of proteins and marker bands are indicated in kilo Dalton $(\mathrm{kDa})$.

In addition, the dimerization and secretion of overexpressed SEMA3A were analyzed. Unspecific binding of the used anti-SEMA3A antibody in unused, concentrated cell culture medium and concentrated cell culture medium of untransfected HEK293 cells was excluded by western blot analysis (data not shown). Single transfections of HEK293 cells with SEMA3A-pCMV-HA plasmids containing the human sequence of SEMA3A WT or of the SEMA3A variants c.196C>T (p.R66W) and c.2002A>G (p.1668V) were performed. $24 \mathrm{~h}$ after transfection, the cell culture medium was concentrated and the cytoplasmic and nuclear protein extracts were separately isolated. Western blot analysis was performed using $30 \mu \mathrm{g}$ of protein per lane treated with the reducing agent DTT (+DTT) or without reducing agent (-DTT). The overexpressed SEMA3A was detected with an anti-SEMA3A antibody. The representative results are shown in Figure 21. In concentrated medium with reducing agent, the secreted monomeric isoforms of $\sim 87 \mathrm{kDa}(\mathrm{p} 87$ ) and $\sim 61 \mathrm{kDa}$ (p61) were detected. In concentrated medium without reducing agent, the secreted dimeric isoforms of $\sim 174 \mathrm{kDa}(\mathrm{p} 87: 87)$ and $\sim 112 \mathrm{kDa}$ (p87:p25) as well as the secreted monomeric isoforms of $\sim 87 \mathrm{kDa}(\mathrm{p} 87)$ and $\sim 61 \mathrm{kDa}(\mathrm{p} 61)$ were received. The secreted monomeric p87-SEMA3A isoform without reducing agent is displayed by a lower protein band possibly due to the missing of DTT. In cytoplasmic and nuclear extracts with reduction, the monomeric isoforms of $\sim 87 \mathrm{kDa}$ (p87) and $\sim 85 \mathrm{kDa}$ (p85) were observed, whereas without reduction a $\sim 170 \mathrm{kDa}$ protein band was identified, potentially representing the dimeric p85:p85-SEMA3A isoform.

Based on these findings, no consequences on the secretion and dimerization of SEMA3A could be identified by comparing HEK293 cells transfected with the SEMA3A WT plasmid and HEK293 cells transfected with the two SEMA3A plasmids with mutation. Thus, the 
impaired SEMA3A secretion due to the p.R66W mutation described by Hanchate et al. (2012) could not be validated.

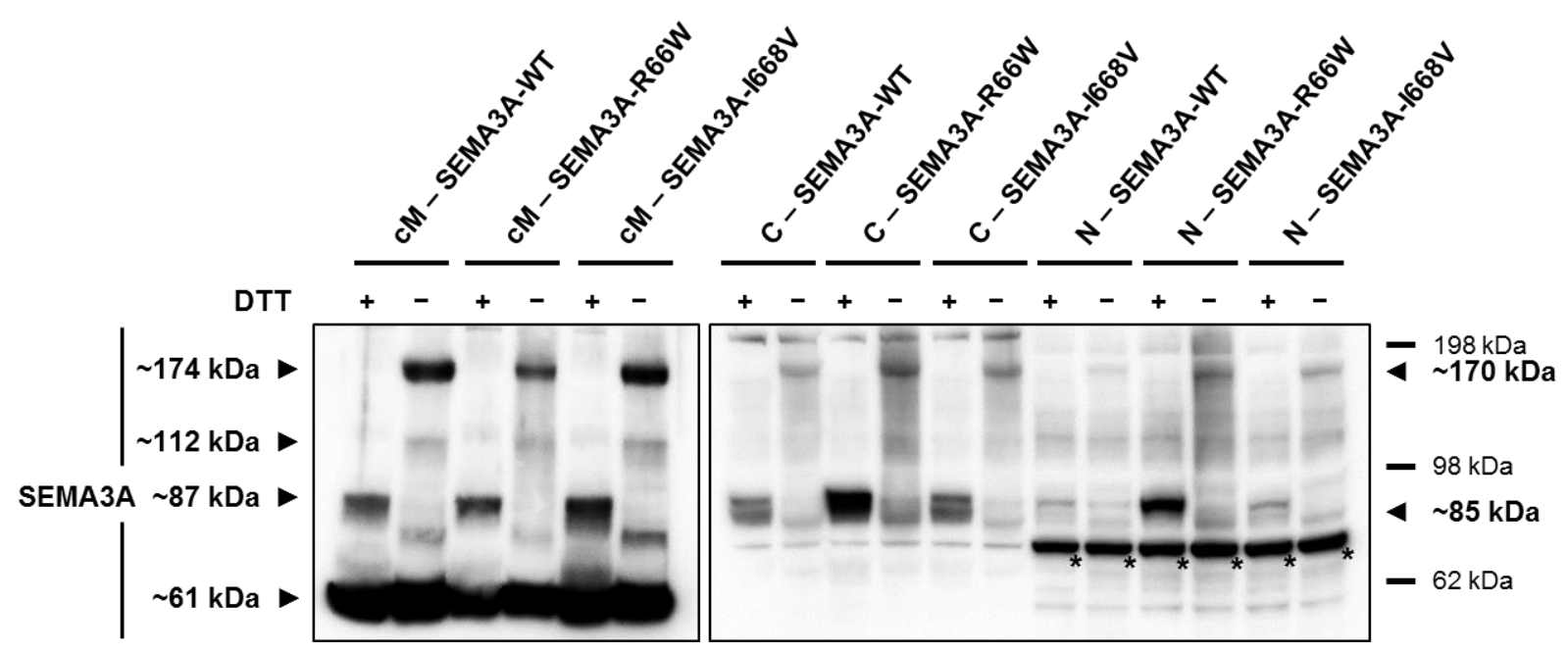

Figure 21: Secretion and dimerization of transient overexpressed SEMA3A WT and SEMA3A variants in HEK293 cells. HEK293 cells were single transfected with SEMA3A-pCMV-HA plasmids containing the human sequence of SEMA3A WT or of the SEMA3A variants C.196C>T (p.R66W) and c.2002A>G (p.1668V). $24 \mathrm{~h}$ after transfection, the cell culture medium was concentrated $(\mathrm{cM})$ and the cytoplasmic $(\mathrm{C})$ and nuclear $(\mathrm{N})$ protein extracts were separately isolated. Western blot analysis was performed with an anti-SEMA3A antibody for the detection of overexpressed SEMA3A proteins. $30 \mu \mathrm{g}$ of protein treated with the reducing agent DTT (+DTT) or without reducing agent (-DTT) was used per lane. In concentrated medium with reduction, the secreted monomeric isoforms of $\sim 87 \mathrm{kDa}$ (p87) and $\sim 61 \mathrm{kDa}$ (p61) were detected. In concentrated medium without reduction, the secreted dimeric isoforms of $\sim 174 \mathrm{kDa}$ (p87:p87) and $\sim 112 \mathrm{kDa}$ (p87:p25) as well as the secreted monomeric isoforms of $\sim 87 \mathrm{kDa}$ (p87) and $\sim 61 \mathrm{kDa}$ (p61) were received. The secreted monomeric p87-SEMA3A isoform without reducing agent is displayed by a lower protein band possibly due to the missing of DTT. In cytoplasmic and nuclear extracts with reducing agent, the monomeric isoforms of $\sim 87 \mathrm{kDa}$ (p87) and $\sim 85 \mathrm{kDa}$ (p85) were detected, whereas without reducing agent a $\sim 170 \mathrm{kDa}$ protein band was identified, potentially representing the dimeric p85:p85-SEMA3A isoform. Consequently, the SEMA3A variants had no effect on the secretion and dimerization of SEMA3A by comparing HEK293 cells transfected with the SEMA3A WT plasmid and HEK293 cells transfected with the two SEMA3A plasmids with mutation. Stars $(*)$ illustrate potentially unspecific protein bands due to unspecific binding of the used antibody. Molecular weights of proteins and marker bands are indicated in kilo Dalton (kDa). 


\subsection{CHD7 co-immunoprecipitates with SEMA3A WT and SEMA3A variants}

Chd7 and Sema3a interact in the nucleus of the murine NCC line 09-1 (chapter 3.5). To investigate this interaction in HEK293 cells and in order to check whether the interaction holds up in presence of SEMA3A mutations, Co-IP experiments were performed. Therefore, HEK293 cells were single transfected with SEMA3A-pCMV-HA plasmids containing the human sequence of SEMA3A WT or of the SEMA3A variants c.196C>T (p.R66W) and c.2002A>G (p.1668V). Nuclear extracts were isolated $24 \mathrm{~h}$ after transfection. The immunoprecipitation of endogenous CHD7 was performed using an anti-CHD7 antibody. Endogenous and overexpressed SEMA3A was detected by western blot analysis using an anti-SEMA3A antibody (Figure 22). A $~ 87 \mathrm{kDa}$ protein band corresponding to the estimated size of the monomeric p87-SEMA3A isoform was detected. Because of the high amount of applied protein in the immunoprecipitation, an interaction of endogenous CHD7 and endogenous SEMA3A was also identified in untransfected HEK293 cells. Nuclear extract of HEK293 cells transfected with SEMA3A WT plasmid without incubation of an anti-CHD7 antibody served as negative control to prove that SEMA3A is not capable to bind unspecific to the magnetic beads used for the Co-IP experiments. $30 \mu \mathrm{g}$ nuclear protein of untransfected and transfected HEK293 cells per lane was used as input and transfection control, validating the SEMA3A overexpression due to the detection of the monomeric isoforms of $\sim 87 \mathrm{kDa}$ (p87) and $\sim 85$ kDa (p85) in nuclear extracts of transfected HEK293 cells.

In summary, the data obtained by the Co-IP experiments reveal an interaction of CHD7 with the overexpressed SEMA3A WT and the overexpressed SEMA3A variants in HEK293 cells. 


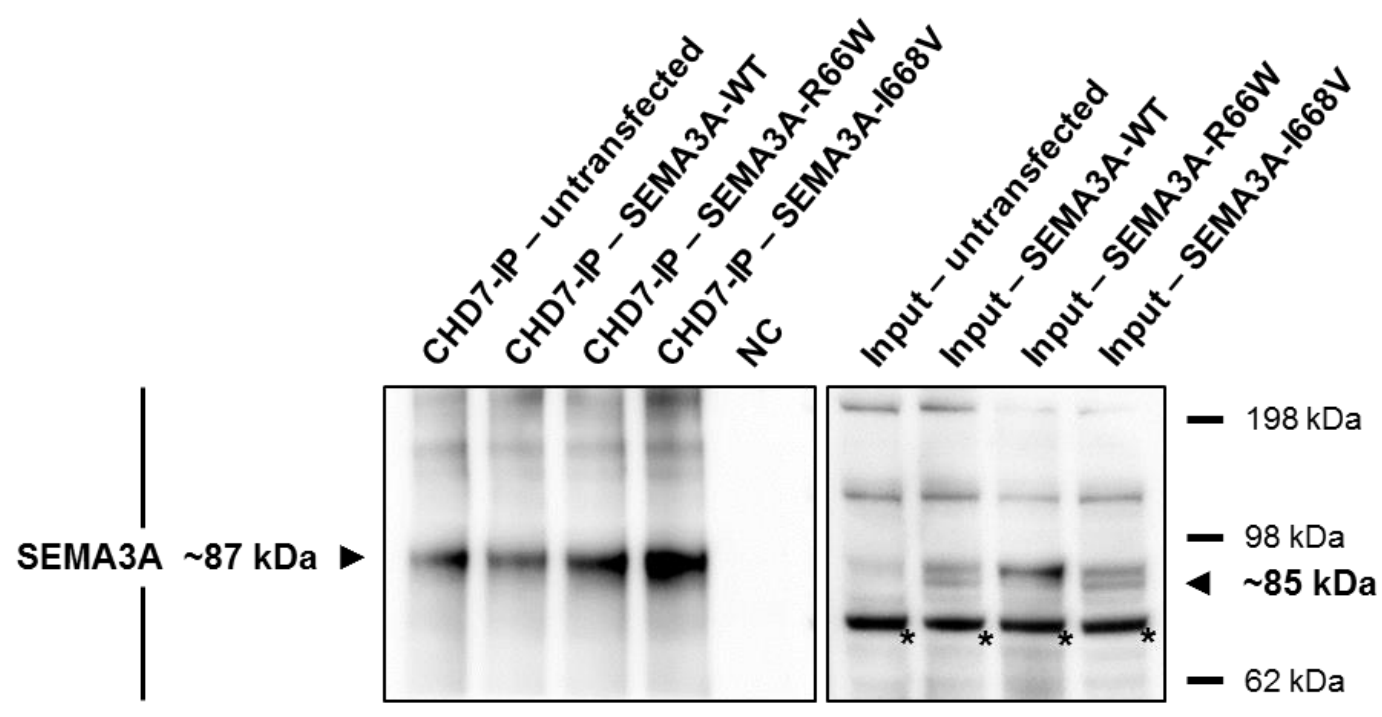

Figure 22: Co-immunoprecipitation of CHD7 with SEMA3A WT and SEMA3A variants in HEK293 cells. HEK 293 cells were single transfected with SEMA3A-pCMV-HA plasmids containing the human sequence of SEMA3A WT or of the SEMA3A variants c.196C>T (p.R66W) and c.2002A $>\mathrm{G}$ (p.1668V). $24 \mathrm{~h}$ after transfection, nuclear protein extracts were isolated. The immunoprecipitation of endogenous $\mathrm{CHD7}$ was performed using an anti-CHD7 antibody. By western blot analysis using an anti-SEMA3A antibody, endogenous and overexpressed SEMA3A was detected. A $\sim 87 \mathrm{kDa}$ (p87) protein band corresponding to the estimated size of the monomeric p87-SEMA3A isoform was observed. An interaction of endogenous CHD7 and endogenous SEMA3A was also identified in untransfected HEK293 cells, because of the high amount of applied protein in the immunoprecipitation. To verify that SEMA3A does not bind unspecific to the magnetic beads used for the Co-IP experiments, nuclear extract of HEK293 cells transfected with SEMA3A WT plasmid without incubation of an anti-CHD7 antibody was used as negative control (NC). $30 \mu \mathrm{g}$ nuclear protein of untransfected and transfected HEK293 cells per lane served as input and transfection control, validating the SEMA3A overexpression due to the detection of the monomeric isoforms of $\sim 87 \mathrm{kDa}$ (p87) and $\sim 85 \mathrm{kDa}$ (p85) in nuclear extracts of transfected HEK293 cells. The Co-IP experiments demonstrate an interaction of CHD7 with the overexpressed SEMA3A WT and the overexpressed SEMA3A variants in HEK293 cells. Stars ( $*$ ) illustrate potentially unspecific protein bands due to unspecific binding of the used anti-SEMA3A antibody. Molecular weights of proteins and marker bands are indicated in kilo Dalton $(\mathrm{kDa})$. 


\subsection{CHD7 shows no direct interaction with SEMA3A WT or SEMA3A variants}

To demonstrate the correct expression of the generated full-length SEMA3A plasmids (chapter 3.7) for yeast two-hybrid assay, yeast cells were single transformed with the HAtag containing plasmids SEMA3A-WT-pGADT7, SEMA3A-R66W-pGADT7 and SEMA3A1668V-pGADT7. Whole yeast cell protein lysates were isolated and analyzed with antibodies against the HA-tag and SEMA3A by western blot analysis. The representative results are shown in Figure 23. The detection of a $\sim 97 \mathrm{kDa}$ protein band corresponding to the estimated size of the generated SEMA3A plasmids indicated no proteolytic cleavage and thus the usability of the plasmids for yeast two-hybrid assay. Whole protein lysate of yeast cells double transformed with CHD7-Cr1-3-pGBKT7 (NP_060250.2) and CHD8-pGADT7-Rec (NP_065971.2) were used as positive control for HA-tag expression ( $70 \mathrm{kDa}$ protein band) as well as negative control for SEMA3A expression (no detectable protein band). The detection of $\alpha$-Tubulin ( $50 \mathrm{kDa}$ protein band) ensured equal protein loading. 


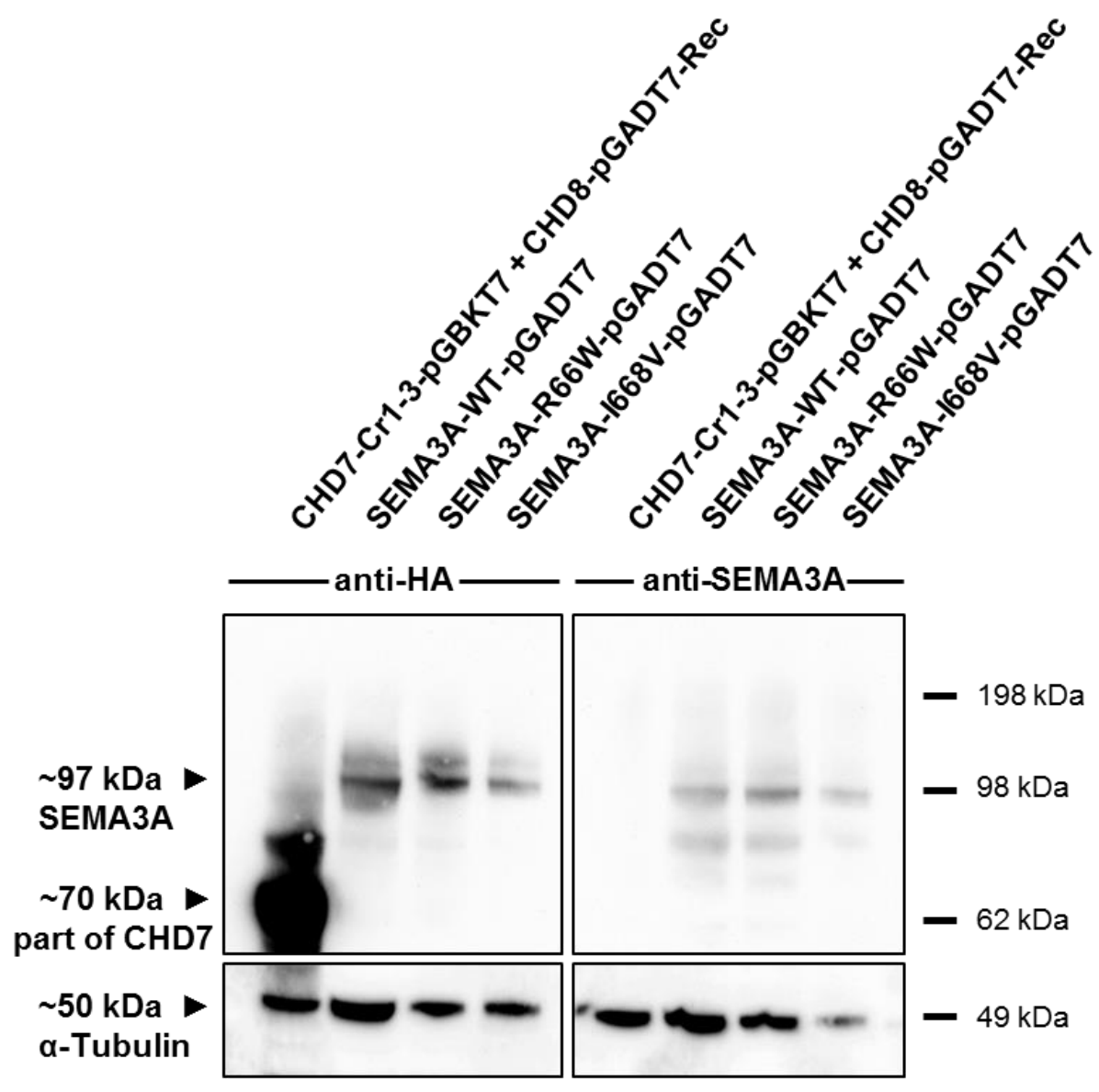

Figure 23: Expression of the full-length SEMA3A isoform in yeast cells. To validate that the SEMA3A yeast-two hybrid plasmids do not undergo proteolytic cleavage, yeast cells were single transformed with the HA-tag containing plasmids SEMA3A-WT-pGADT7, SEMA3A-R66W-pGADT7 and SEMA3A-1668V-pGADT7. Whole yeast cell protein lysates were incubated with an anti-HA antibody (upper, left panel) or an anti-SEMA3A antibody (upper, right panel). By western blot analysis, a $\sim 97 \mathrm{kDa}$ protein band corresponding to the estimated size of the generated SEMA3A plasmids was detected, indicating no proteolytic cleavage and thus the usability of the plasmids for yeast two-hybrid assay. Whole protein lysate of yeast cells double transformed with CHD7-Cr1-3-pGBKT7 (NP_060250.2) and CHD8-pGADT7-Rec (NP_065971.2) were used as positive control for HA-tag expression ( $\sim 70 \mathrm{kDa}$ protein band) as well as negative control for SEMA3A expression (no detectable protein band). Lower panel: Detection of $\alpha$-Tubulin ( $50 \mathrm{kDa}$ protein band) ensured equal protein loading. Molecular weights of proteins and marker bands are indicated in kilo Dalton $(\mathrm{kDa})$.

To determine a direct interaction of CHD7 with SEMA3A, yeast two-hybrid assay was performed. Four CHD7 plasmids were available as bait plasmids namely CHD7-1-pGBKT7 (amino acids 1-799), CHD7-2-pGBKT7 (amino acids 732-1567), CHD7-3-pGBKT7 (amino acids 1533-2380) and CHD7-4-pGBKT7 (amino acids 
2325-2997) (Schulz et al., 2014a). These plasmids divide the human full length CHD7 protein into four overlapping fragments without disruption of a known functional domain (Figure 24). Owing to autoactivation of the CHD7-1-pGBKT7 plasmid in the yeast system, no yeast two-hybrid assay could be performed with this plasmid (Schulz et al., 2014a). In addition, the CHD7-Cr1-3-pGBKT7 plasmid was used as bait, spanning the amino acids 1591-2181 (Figure 24) (Batsukh et al., 2010). All appropriate bait plasmids were used with the prey plasmids SEMA3A-WT-pGADT7, SEMA3A-R66W-pGADT7 and SEMA3A-I668VpGADT7 for yeast two-hybrid assay, respectively. The plasmid SEMA3A-R66W-pGADT7 contain the already known SEMA3A missense mutation p.R66W (Hanchate et al., 2012), whereas the plasmid SEMA3A-I668V-pGADT7 include the missense mutation identified in a CHD7-positive CHARGE syndrome patient (chapter 3.6). Yeast colonies on -LT agar plates indicated a successful transformation (Figure $25 \mathrm{~A}, \mathrm{C}$ and E). No autoactivation of the yeast reporter gens could be observed on -LTHA agar plates using an empty pGBKT7 bait vector together with the prey plasmids in an autoactivation test, respectively (Figure 25 B, D and F). As positive control, the bait plasmid CHD7-Cr1-3-pGBKT7 and the prey plasmid CHD8-pGADT7-Rec (amino acids 1789-2091) were used (Batsukh et al., 2010) (Figure $25 \mathrm{~B}, \mathrm{D}$ and F). The yeast two-hybrid assay with the SEMA3A plasmids SEMA3A-WT-pGADT7, SEMA3A-R66W-pGADT7 and SEMA3A-I668V-pGADT7 revealed no direct interactions with the plasmids CHD7-2-pGBKT7, CHD7-3-pGBKT7, CHD7-4-pGBKT7 and CHD7-Cr1-3-pGBKT7 (Figure 25 B, D and F), demonstrating that these parts of CHD7 do not directly bind to SEMA3A WT or SEMA3A variants.

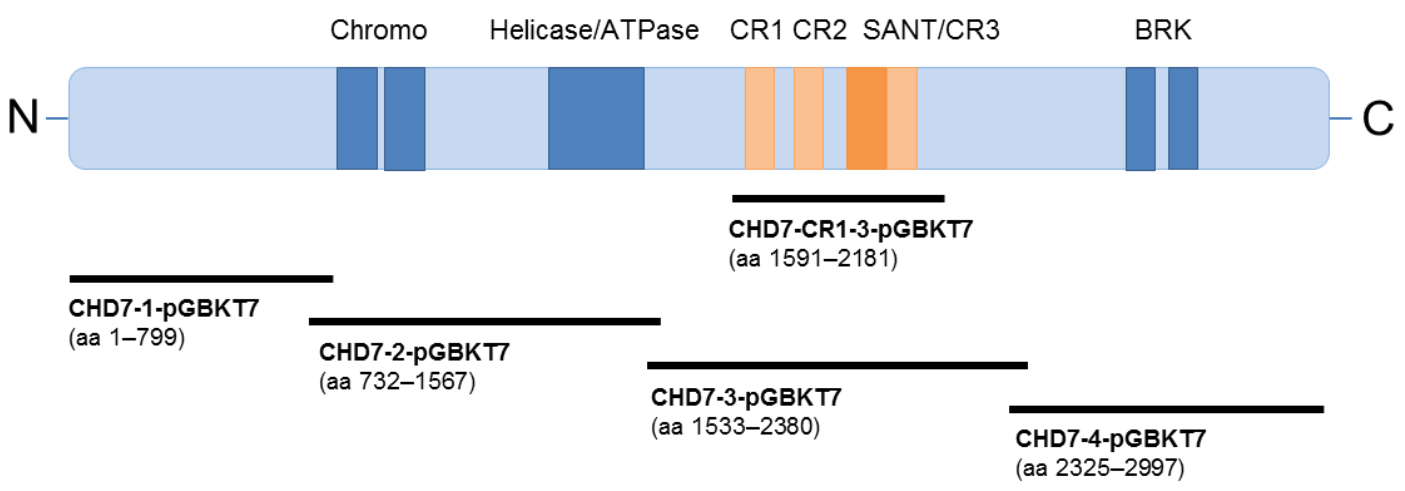

Figure 24: Schematic overview of the used CHD7 yeast two-hybrid assay plasmids. The plasmids CHD7-1-pGBKT7 to CHD7-4-pGBKT7 divide the full-length CHD7 protein into four overlapping parts. A known functional domain is thereby not disrupted. The plasmid CHD7-Cr1-3pGBKT7 achieves this requirement as well. Schulz et al. (2014a). 


\section{$-\mathrm{LT}$}

A



C

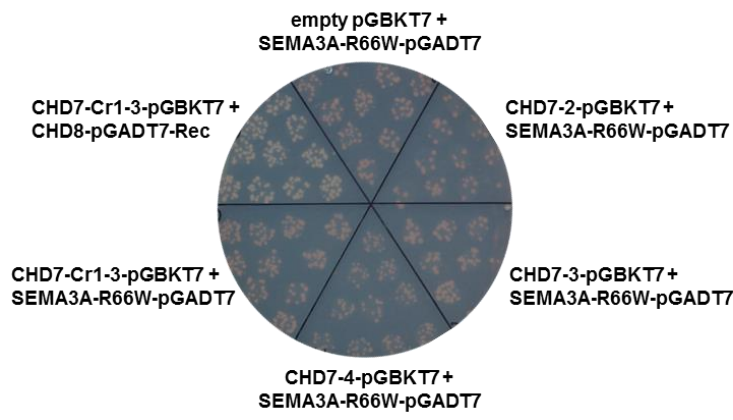

E



-LTHA

B



D

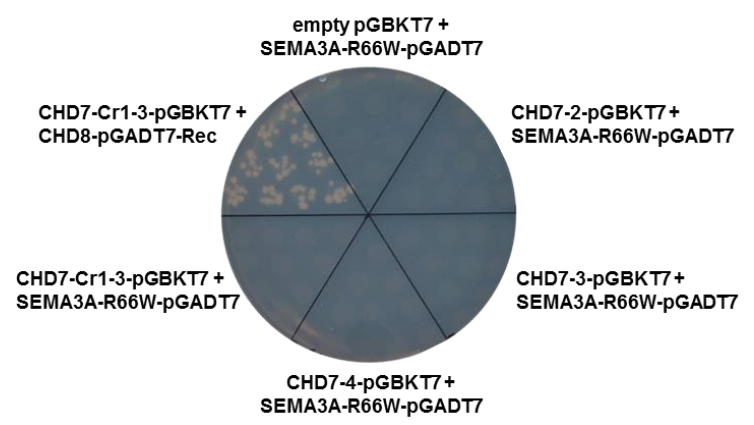

$\mathbf{F}$

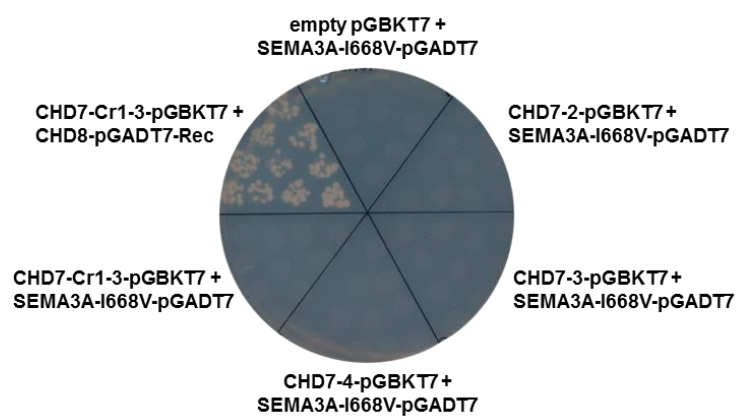

Figure 25: Yeast two-hybrid assay. Yeast two-hybrid assay was performed with the plasmids CHD7-2-pGBKT7, CHD7-3-pGBKT7, CHD7-4-pGBKT7 and CHD7-Cr 1-3-pGBKT7 as baits, which are parts of the human full-length CHD7 protein. The plasmids SEMA3A-WT-pGADT7, SEMA3AR66W-pGADT7 and SEMA3A-I668V-pGADT7 were used as preys. Yeast colonies on -LT agar plates indicated a successful transformation $(\mathbf{A}, \mathbf{C}$ and $\mathbf{E}$ ). All prey plasmids were tested on -LTHA agar plates for autoactivation of the Y2HGold yeast strain reporter gens with the empty PGBKT7 bait vector (B, D and F). As positive control, the bait plasmid CHD7-Cr1-3-pGBKT7 and the prey plasmid CHD8-pGADT7-Rec were used (B, D and F). The yeast two-hybrid assay revealed no direct interaction of the CHD7 parts with the SEMA3A WT or the SEMA3A variants (B, D and F). 


\section{$4 \quad$ Discussion}

\subsection{Summary of the results}

Using RT-PCR analysis, the two different murine NCC lines JoMa and 09-1 were characterized for the expression of different marker genes and Chd7. The nuclear expression of Chd7 was validated in both NCC lines by western blot analysis and in the O9-1 cells also with immunocytochemical staining. The results of the western blot analysis revealed a strong Chd7 expression in 09-1 cells in comparison with JoMa cells. Based on these findings, further experiments were performed with 09-1 cells. With RT-PCR analysis it was further shown that 09-1 cells express beside the receptors of class 3 semaphorins also class 3 semaphorins itself. The nuclear and cytoplasmic Sema3a expression was confirmed by immunocytochemical staining and western blot analysis. Moreover, proteolytic processing, dimerization and secretion of Sema3a were identified using western blot analysis. A downregulation of $C h d 7$ with specific siRNAs revealed a reduced Sema3a expression in 09-1 cells. Using Co-IP experiments, the nuclear interaction of Chd7 with Sema3a was shown in two ways: by precipitation of Chd7 and detection of Sema3a as well as by precipitation of Sema3a and detection of Chd7.

A mutational screen of the coding sequence of SEMA3A in 5 CHARGE patients with a mild phenotype and 10 CHARGE patients with a severe phenotype, but all carrying pathogenic mutations in the CHD7 gene, was performed. In one patient, the nonsynonymous variant c.2002A $>G$ (p.l668V) was identified in a heterozygous state. This new identified variant, as well as the already published variant p.R66W, were characterized in the human HEK293 cell line using overexpressing SEMA3A plasmids containing one of the mutations, respectively. Western blot analyses demonstrated that expression, proteolytic processing and dimerization as well as secretion of SEMA3A were not affected by both mutations. The performed Co-IP experiments revealed that CHD7 interacts with SEMA3A WT and the two SEMA3A variants, p.R66W and p.I668V. However, yeast two-hybrid assay identified no direct interaction of the used CHD7 fragments with full-length constructs of wildtype or mutated SEMA3A. 


\subsection{NCCs - which in vitro model to choose?}

The symptoms seen in CHARGE syndrome patients likely predominantly result from defects of the cranial NCCs and their subpopulation, the cardiac NCCs, cell populations largely responsible for forming the vertebrate head, heart and the peripheral nervous system (Kulesa et al., 2010). The assumption that the malformations and defects seen in CHARGE syndrome patients result from abnormalities during NCC development were already published in 1985 (Siebert et al., 1985). Furthermore, defects in the interaction between mesoderm and NCCs (Van Meter and Weaver, 1996) or disturbances in mesenchymal-epithelial interactions (Williams, 2005) were also supposed as pathogenic mechanisms causing CHARGE. In 2004, Vissers et al. identified mutations in the CHD7 gene as the underlying cause leading to the complex developmental disorder CHARGE syndrome (Vissers et al., 2004). In several other studies, further heterozygous mutations or deletions of $\mathrm{CHD7}$ were described in CHARGE syndrome patients (Aramaki et al., 2006; Jongmans et al., 2006; Lalani et al., 2006; Sanlaville et al., 2006; Wincent et al., 2008). CHD7 was also identified as a member of complexes which regulate time and tissue specific gene expression (Layman et al., 2010). The initial presumption that CHARGE belongs to the neurocristopathies was confirmed by Bajpai et al. (2010) for the first time. Performing Chd7 knockdown experiments in Xenopus laevis embryos, Bajpai et al. (2010) demonstrated that Chd7 is essential for the formation of NCCs by activating transcriptions factors such as Snai2 (previously named Slug), Sox9 and Twist and observed abnormal NCC migration into the pharyngeal arches. Bajpai et al. (2010) also identified the association of Chd7 with the PBAF complex and supposed that this cooperation regulates the gene expression during NCC formation in Xenopus laevis.

A suitable NCC model for in vitro studies was essential to investigate the functions of Chd7 in NCC. To examine that these cell line truly represents NCC and express Chd7, the two murine NCC lines, the trunk JoMa cells and the cranial 09-1 cells, were characterized by RT-PCR and western blot analysis as well as immunocytochemical staining. Both cell lines grow as monolayers and display a stellate like morphology, typical for NCCs (Rao and Anderson, 1997). Furthermore, they can be cultured in a suitable culture medium without loss of their self-renewal capacity and multipotency. Maurer et al. (2007) generated the immortalized cell line JoMa1 and a clonally derived cell line termed JoMa1.3 using transgenic mice (Jager et al., 2004) at E8.75, expressing a conditional 4-Hydroxytamoxifen (4-OHT) inducible allele of c-Myc (c-MycER ${ }^{\top}$; Pelengaris et al., 1999; Rudolph et al., 2000). JoMa cells proliferate robustly as long as c-MycER ${ }^{\top}$ is active in a 
4-OHT dependent manner (Maurer et al., 2007). They were described to express amongst others the two NCC markers Sox10 and Ngfr (previously named P75), indicating that these cells have the potential to differentiate into various cell types in vivo (Maurer et al., 2007). A detailed look at the RT-PCR primers used by Maurer et al. (2007) for the expression analysis of marker genes in JoMa cells revealed that several primers were not useful for RT-PCR analysis due to recognition sites in intronic sequences. For this reason, the Sox10 expression in JoMa cells described by Maurer et al. (2007) is questionable. Maurer et al. (2007) successfully differentiated both JoMa cell lines into neurons, glia, melanocytes, smooth muscle cells and chondrocytes in vitro. The second murine NCC line, the 09-1 cells, was generated by Ishii et al. (2012). This cell line was obtained from mass culture of Wnt1-Cre;R26R-GFP reporter-expressing NCCs from mouse embryos at E8.5 (Ishii et al., 2012). Whole-genome expression profiling suggested that this cell line stably expresses both NCC and stem cell markers and was successfully differentiated into osteoblasts, chondrocytes, smooth muscle cells and glia (Ishii et al., 2012).

By RT-PCR analysis on cDNA obtained from total RNA of JoMa and 09-1 cells the initial situation of both NCC lines was monitored concerning the expression of different marker genes and Chd7. In contrast to the tested NCC markers Ngfr, Snai2, Pax3, Snai1 and Twist1, the NCC marker Sox10 was not detected in both cell lines, indicating JoMa and 09-1 cells are no pre-migratory cell lines. The further tested marker genes for neurons, glial cells, smooth muscle cells, melanocytes and chondrocytes displayed in both NCC lines a similar expression pattern. The additionally analyzed Chd7 gene was found to be expressed in both NCC lines. To verify the results of the Chd7 expression on protein level, nuclear protein extracts of JoMa cells and 09-1 cells were tested by western blot analysis. The expression of Chd7 could be confirmed in 09-1 cells, but not in JoMa cells because no clear protein band of Chd7 was detected. By immunocytochemical staining it was shown that Chd7 is specifically and intensely expressed in the nucleus of 09-1 cells. The strong Chd7 expression strengthens the hypothesis that the symptoms seen in CHARGE syndrome can be explained by a dysfunction of the cranial NCCs and not of the trunk NCCs. For this reason, further experiments were performed with the cranial 09-1 cell line, representing multipotent mesenchymal cranial NCCs. 


\subsection{NCCs express class 3 semaphorins and secrete Sema3a}

Randall et al. (2009) demonstrated that the phenotype of pharyngeal arch artery defects in Chd7 heterozygous mice could not be rescued by expression of Chd7 in the murine NCCs alone, whereas Chd7 expression in both NCCs and the pharyngeal ectoderm led to a full rescue of the pharyngeal arch artery alterations. As candidate genes for the interaction between the ectoderm and the NCCs, semaphorins, ephrins, slits, Pdgfs and Vegfs were suggested (Randall et al., 2009). To identify Chd7 target genes in different tissues with functions in NCC development and possible other pathways which might also contribute to the phenotype of CHARGE syndrome, Schulz et al. (2014b) performed a genome-wide microarray expression analysis on whole wild type and Chd7 deficient (Chd7 ${ }^{\text {Whilt }}$ and Chd $7^{\text {WhiWhi }}$ ) embryos of the Whirligig mouse line at E9.5 and identified 98 differentially expressed genes. Many of the misregulated genes are known to play a role in NCC migration and axon guidance like class 3 semaphorins and ephrins as well as transcription factors, overall genes belonging to the candidate molecules for the interaction between the ectoderm and the NCCs assumed by Randall et al. (2009).

During development, NCCs are guided to their appropriate target regions through the embryo by many attractive and repulsive cues. A group of guidance cues that is well investigated are the semaphorins which exert their effects through the binding to multimeric receptor complexes at the cell membrane and initiating of unique intracellular signal transduction cascades (Jongbloets and Pasterkamp, 2014).

To test whether the NCC line 09-1 expresses semaphorin receptors, RT-PCR analysis on cDNA obtained from total RNA of 09-1 cells was performed, representing expression of neuropilin and plexin receptors. Plein et al. (2015) performed Sema3c in situ hybridization (ISH) on outflow tracts of mouse embryos and identified Sema3c expression in myocardial cuff cells and in the area where cardiac NCCs were located. In contrast to prior studies, who interpreted this result as an indication that Sema3c guide cardiac NCCs into this area, Plein et al. (2015) suggested that cardiac NCCs express Sema3c itself. By performing a Sema3c ISH on Wnt1-Cre Rosa ${ }^{Y f p}$ outflow tracts, they found that Sema3c was expressed in a subpopulation of cardiac NCCs. Therefore, they supposed that semaphorins might act not only as guidance cues, but also have additional functions (Plein et al., 2015). After the validation of the semaphorin receptor expression in 09-1 cells, which are essential for the guidance of the semaphorins, a possible semaphorin expression in NCC itself was investigated. RT-PCR analysis on CDNA of 
O9-1 cells identified the expression of class 3 semaphorins, in particular Sema3a, Sema3c, Sema3d and Sema3e, in NCCs for the first time.

These results raised several questions:

1. In which cell compartments of NCCs are class 3 semaphorins localized?

2. Exist class 3 semaphorins as monomeric and dimeric isoforms in NCCs?

3. Undergo class 3 semaphorins proteolytic processing in NCCs?

4. Are class 3 semaphorins secreted by NCCs?

For SEMA3A, a secreted class 3 semaphorin with repulsive effects on primary olfactory axons, a contribution to the phenotype of Kallmann syndrome was demonstrated (Hanchate et al., 2012; Young et al., 2012). Phenotypic features of the heterogeneous congenital disorder Kallmann syndrome, like a hypogonadotropic hypogonadism and an absent or impaired sense of smell (anosmia) (Dodé and Hardelin, 2009), are also frequently observed in patients with CHARGE syndrome (Chalouhi et al., 2005; Pinto et al., 2005; Ogata et al., 2006). In addition, mutations in the CHD7 gene can be found in patients with CHARGE and in a minority of patients with Kallmann syndrome (Kim et al., 2008; Bergman et al., 2011). For this reason, the Kallmann syndrome was suggested to represent the mild end of the phenotypic spectrum of CHARGE syndrome (Jongmans et al., 2009). Consequently, if $C H D 7$ and SEMA3A play a role in the pathogenesis of Kallmann syndrome, it might be possible that beside CHD7 additional SEMA3A contributes to the pathogenesis of CHARGE syndrome. Therefore, Sema3a was further investigated in the NCC line 09-1 by immunocytochemical staining and western blot analysis. Sema3a localization was not only identified in concentrated culture medium, exposing secretion of Sema3a by 09-1 cells, but also in the cytoplasm and nucleus of 09-1 cells. The localization of Sema3a in the nucleus is not due to a nuclear localization signal which is not present in Sema3a. It could be explained by glycosylation of the Sema3a isoforms (Rondanino et al., 2003). The isoforms detectable with the used anti-Sema3a antibody displayed full-size and partially processed monomers and dimers, indicating proteolytic cleavage of the full-length Sema3a by furin and furin-like endoproteases or matrix metalloproteases and dimerization as described for related class 3 semaphorins (Adams et al., 1997; Klostermann et al., 1998; Koppel and Raper, 1998; Christensen et al., 2005; Esselens et al., 2010). Processing of the inactive fulllength Sema3a isoform is described to result in activation of its repulsive properties and 
generation of functionally different isoforms (Adams et al., 1997). Adams et al. (1997) further assumed that specific proteases and protease inhibitors might locally activate or modulate repulsive signals and consequently contribute to class 3 semaphorin specificity. Nevertheless, further experiments are needed to clarify these aspects.

In conclusion, the experiments demonstrate that NCCs express, process and secrete Sema3a, providing Sema3a itself. These results lead to the suggestion that Sema3a has in addition to its NCC guidance aspect other diverse functions.

\subsection{Chd7 regulates Sema3a expression and is associated with Sema3a in NCCs}

Chd7 is highly conserved across species and orthologs have been identified in mouse, chicken, zebrafish, Xenopus laevis and others (Bosman et al., 2005; Aramaki et al., 2007; Bajpai et al., 2010). A downregulation of Chd7 in Xenopus laevis embryos displayed major features seen in CHARGE syndrome patients (Bajpai et al., 2010) and led to induction and migration defects of NCCs (Bajpai et al., 2010; Schulz et al., 2014b), while downregulation of Sema3a in Xenopus laevis embryos only affected NCC migration (Schulz et al., 2014b). Sema3a expression analysis in heterozygous (Chd7 ${ }^{\text {Whi/t }}$ ) as well as homozygous (Chd $7^{\text {Whi/Whi}}$ ) Whirligig mice and after downregulation of Chd7 in Xenopus laevis embryos revealed a decreased or complete depletion of Sema3a expression exposing the evolutionary conservation of the Chd7-Sema3a regulatory loop (Schulz et al., 2014b).

To analyze whether this regulatory mechanism is also found in NCCs, knockdown experiments were performed by transfecting 09-1 cells with Chd7-specific siRNAs. Analysis of Sema3a expression after downregulation of Chd7 revealed a decreased expression of Sema3a, demonstrating that the regulatory loop is also present in NCCs.

Co-IP experiments performed with 09-1 cells further demonstrated an interaction of Chd7 with Sema3a in nuclear extract of NCCs suggesting an association of Chd7 and Sema3a in the same protein complex. Chd7 rescue experiment performed by co-injection of a Chd7 morpholino and Sema3a RNA demonstrated a partially rescue of the Chd7 knockdown phenotype seen in Xenopus laevis embryos (Borchers, unpublished data), indicating a possible role of Sema3a in the pathogenesis of CHARGE syndrome. 


\subsection{SEMA3A might act as modifier in CHARGE syndrome}

High clinical inter- and intra-familial variability is observed in the malformation disorder CHARGE syndrome (Aramaki et al., 2006; Jongmans et al., 2006; Lalani et al., 2006). Genetic modifiers make an important contribution to different kinds of disease like neurological (Kearney, 2011) or arrhythmogenic disorders (Crotti et al., 2005). Modifier can enhance or suppress the phenotype of a disorder. To analyze whether SEMA3A mutations contribute to the pathogenesis of CHARGE syndrome and maybe negatively modulate the phenotypic outcome of the affected patients, 5 patients with a mild and 10 patients with a severe phenotype, but all carrying a pathogenic mutation in the CHD7 gene, were sequenced for the presence of additional mutations in the coding sequence of the SEMA3A gene.

One non-synonymous mutation in a heterozygous state, namely c.2002A $>G$ (p.1668V) was identified in a patient with a severe phenotype. This variant is not listed in the database of the Exome Aggregation Consortium (ExAC). While two in silico programs predicted this variant to be tolerated (PolyPhen-2 and SIFT), the in silico program MutationTaster predicted the variant as disease causing. Interestingly, Schulz et al. (2014b) screened 45 patients diagnosed with CHARGE, but lacking a mutation in the CHD7 gene for mutations in SEMA3A and identified also the non-synonymous variant c. $2002 A>G$ (p.1668V) in one patient and two other non-synonymous variations namely c.196C>T (p.R66W) and c.2062A>G (p.T688A) in a heterozygous state in two other patients. Two of the later found mutations were already described in patients with Kallmann syndrome leading to an impaired SEMA3A secretion (p.R66W) or a reduced signal activity of SEMA3A (p.T688A) (Hanchate et al., 2012). Based on these findings, a pathogenic effect of SEMA3A in Kallmann syndrome was assumed (Hanchate et al., 2012). Nevertheless, Hanchate et al. (2012) supposed that heterozygous SEMA3A mutations are not sufficient to induce the phenotype of Kallmann syndrome alone, but might contribute to it by a combination with other mutated Kallmann syndrome causing gene mutations due to digenetic inheritance. This hypothesis was fleshed out by identification that the non-synonymous mutation p.R66W was inherited from the healthy father of a CHARGE syndrome patient (Schulz et al., 2014b) and can be further strengthen because of two independently described patients with bi-allelic mutations in SEMA3A causing an autosomal recessive type of syndromic short stature (Hofmann et al., 2013; Baumann et al., 2017). 
Functional analyses on transfected HEK293 cells were performed to analyze the effect of the new identified SEMA3A mutation p.I668V in a CHD7-positive CHARGE syndrome patient. Therefore, the mutation was induced by side-directed mutagenesis into a plasmid containing the human SEMA3A WT sequence to generate an overexpression plasmid with the defect and to study the effect of the new SEMA3A mutation. As referees, overexpression plasmids containing either the already described SEMA3A mutation p.R66W or the SEMA3A WT sequence were used. With the performed western blot analyses, no alterations concerning the expression, processing or dimerization as well as secretion of SEMA3A were detected by the mutations p.R66W and p.1668V. These results indicate that the p.R66W mutation might has no effect on the secretion of SEMA3A as assumed by Hanchate et al. (2012). However, the results obtained by Hanchate et al. (2012) were limited by the analysis of only conditioned medium without transfection control. During writing this thesis, my research group studied the effect of tunicamycin, a glycosylation inhibitor, on the glycosylation of overexpressed SEMA3A in HEK293 cells using overexpressing plasmids containing either the SEMA3A WT or the SEMA3A mutations p.R66W and p.I668V. The results of the western blot analysis revealed that after tunicamycin treatment of transfected HEK293 cells, in nuclear and cytoplasmic extracts, which contained the overexpressed SEMA3A WT and the overexpressed SEMA3A with the mutation p.I668V, only unglycosylated SEMA3A was detected. Indeed, in nuclear and cytoplasmic extracts containing the overexpressed SEMA3A with the mutation p.R66W, unglycosylated as well as glycosylated SEMA3A was received. These results might be explained due to structural modifications of SEMA3A caused by the p.R66W mutation. Sema3a rescue experiments in Xenopus laevis embryos performed by co-injection of a Chd7 morpholino with Sema3a-WT, Sema3a-p.R66W or Sema3ap.1668V RNA supposed that only the Sema3a-p.R66W RNA cannot compensate the defects produced by the Chd7 morpholino (Borchers, unpublished data). These data strengthen the hypothesis that the p.R66W mutation is pathogenic, whereas the pathogenicity of the p.1668V mutation could not be clarified. Nevertheless, as presumed in previous studies, heterozygous SEMA3A mutations alone are not sufficient to cause a pathogenic phenotype, but might act as modifier or contribute to it through their interactions with other mutated genes due to digenetic inheritance. In addition, Sema3a was identified in this thesis to be expressed and secreted by the NCC line 09-1 suggesting a new function of SEMA3A. Although Co-IP experiments on transfected HEK293 cells indicated a co-existence of CHD7 and SEMA3A in the same protein 
complex, yeast two-hybrid assay revealed no direct interaction for CHD7 and SEMA3A. The association of CHD7 and SEMA3A in a nuclear protein complex might indicate a function for SEMA3A as transcription factor beside the guidance function as described by Kuriyama and Mayor (2008). Moreover, another effectiveness of Sema3a might be possible, because it is secreted by NCCs itself and not only by the surrounding tissue.

\subsection{Future perspectives}

The Sema3a expression analysis in the NCC line 09-1 revealed the existence of Sema3a not only in the secreted medium, but also in the cytoplasm and in the nucleus of the cells although Sema3a is predicted not to have a nuclear localization signal. To test the accuracy of the detected endogenous Sema3a in 09-1 cells and furthermore of the overexpressed SEMA3A in HEK293 cells, plasmids containing a detectable HA-tag with and without mutation should be used for transfection and western blot analysis. My research group already started with these experiments during writing my thesis.

In order to identify the precise localization of Sema3a in the cytoplasm as well as in the nucleus, a co-immunostaining using different cell organelle markers could be performed.

To elucidate Sema3a target genes in NCCs, the expression profiles of 09-1 cells treated with and without siRNA against Sema3a should be compared by whole-genome microarray analysis. The identified genes could be validated by qRT-PCR analysis and chromatin immunoprecipitation (ChIP) assay.

To confirm the identified association of Chd7 and Sema3a in the same nuclear protein complex in 09-1 cells, Duolink proximity ligation assay (PLA) should be performed. Further, the composition of the whole protein complex should be analyzed. Therefore, a chemical crosslinking in combination with mass spectrometry could be used to examine the structure and composition of the likely heterogenic complex.

To identify Sema3a target genes in NCCs, the expression profiles of 09-1 cells treated with and without siRNA against Sema3a should be compared by whole-genome microarray analysis.

NCCs are guided by semaphorins, but also express semaphorins themselves as demonstrated in the present thesis. Using a migration assay, the influence of downregulated or mutated Sema3a as well as Chd7 on the migration of NCCs could be examined. 
In this thesis it is supposed that mutated SEMA3A might act as modifier in the phenotypic variable malformation syndrome CHARGE contributing to a more severe phenotype of the affected patients. To strengthen this hypothesis further mild and severe affected CHARGE syndrome patients carrying a pathogenic $\mathrm{CHD7}$ mutation should be screened for additional mutations in the SEMA3A gene. Possible identified new SEMA3A mutations as well as already known SEMA3A mutations either assumed to contribute to the phenotype of Kallmann syndrome (Hanchate et al., 2012; Young et al., 2012) or causing an autosomal recessive type of syndromic short stature (Hofmann et al., 2013; Baumann et al., 2017) should be investigated by functional analysis as performed in this thesis. Moreover, the Xenopus laevis model could be used for further functional analyses regarding selected mutations. After a Sema3a knockdown using Sema3a morpholinos, other selected mutations could be used for rescue experiments to analyze the effect of the mutations. 


\section{$5 \quad$ Summary}

CHARGE syndrome is a complex congenital malformation disorder, caused in more about two-thirds of the cases by heterozygous loss of function mutations in the chromodomain helicase DNA-binding protein 7 (CHD7). The acronym CHARGE reflects the main clinical features seen in the most CHARGE syndrome patients namely coloboma, heart defects, atresia of the choanae, retarded growth and development, genital hypoplasia and ear anomalies/deafness. Because of the phenotypic spectrum, it was supposed that the CHARGE syndrome belongs to the neurocristopathies. In addition, the essential role of CHD7 for the formation and migration of neural crest cells (NCCs), which give rise to many tissues of the embryo including craniofacial and heart structures, was recently demonstrated.

Two murine NCC lines, the trunk JoMa cells and the cranial 09-1 cells, were characterized for the expression of NCC and other molecular marker genes as well as Chd7 by RT-PCR analysis. The results revealed that the two cell lines do not differ much in the expression of the tested marker genes for NCCs, neurons, glial cells, smooth muscle cells, melanocytes and chondrocytes as well as Chd7. The nuclear expression of Chd7 was validated by western blot analysis and for 09-1 cells also by immunocytochemical staining. The results of the western blot analysis demonstrated a strong Chd7 expression in 09-1 cells in comparison with JoMa cells. This strengthens the assumption that the symptoms seen in CHARGE syndrome are caused by a dysfunction of the cranial NCCs and not of the trunk NCCs.

Proper NCC guidance through the embryo depends on guidance cues and the large family of the semaphorins thereby plays an important role. Subsequent experiments were performed with the NCC line 09-1. Using RT-PCR analysis, the expression of semaphorin receptors and for the first time of class 3 semaphorins was identified in NCCs. Sema3a expression was proven by immunocytochemical staining and western blot analysis. Further experiments were carried out to check the localization of Sema3a in the different cell compartments and a possible Sema3a secretion into the cell culture medium. Western blot analysis revealed both the expression in the nucleus and the cytoplasm and the secretion into the medium. Moreover, the proteolytic cleavage and dimerization of Sema3a could be identified as described for class 3 semaphorins. Recently, the described regulatory effect of Chd7 on Sema3a in Xenopus laevis embryos and mouse embryos could be confirmed in 09-1 cells through the Sema3a downregulation by siRNA-mediated 
depletion of Chd7. Further experiments revealed a co-immunoprecipitation of $\mathrm{Chd7}$ with Sema3a, indicating the association of these two proteins in the same protein complex.

A further part of the present thesis focused on the identification of possible mutations in the SEMA3A gene of CHD7-positive CHARGE syndrome patients. SEMA3A mutations are hypothesized to negatively modulate the phenotype of CHARGE patients and are known to be involved in the pathogenesis of the allelic disorder Kallmann syndrome, which is suggested to represent the mild end of the phenotypic abnormalities seen in CHARGE syndrome. The non-synonymous SEMA3A mutation c.2002A>G (p.1668V) was identified in a severe affected CHARGE syndrome patient. This finding might strengthens the hypothesis that SEMA3A act as modifier in CHARGE patients with a pathogenic mutation in the CHD7 gene contributing to a more severe phenotype in these patients due to digenetic inheritance. It would also explain the high clinical inter- and intra-familial variability of CHARGE syndrome. The new identified mutation and the already published variant p.R66W were characterized with overexpressing plasmids in the human HEK293 cell line on protein level. The obtained results showed no effect of the mutations on expression, proteolytic processing, dimerization or secretion of SEMA3A. By co-immunoprecipitation experiments it was demonstrated that $\mathrm{CHD} 7$ interacts with SEMA3A WT and the two SEMA3A variants, p.R66W and p.I668V. Yeast-two hybrid assay indeed revealed no direct interaction between CHD7 and SEMA3A with or without mutation.

In summary, the results of the present thesis are another step towards understanding the pathomechanism behind the neurocristopathy CHARGE syndrome. 


\section{$6 \quad$ References}

Acloque H, Adams MS, Fishwick K, Bronner-Fraser M, Nieto MA (2009). Epithelialmesenchymal transitions: the importance of changing cell state in development and disease. The Journal of clinical investigation 119(6): 1438-1449.

Adams RH, Lohrum M, Klostermann A, Betz H, Puschel AW (1997). The chemorepulsive activity of secreted semaphorins is regulated by furin-dependent proteolytic processing. The EMBO journal 16(20): 6077-6086.

Aramaki M, Kimura T, Udaka T, Kosaki R, Mitsuhashi T, Okada Y, Takahashi T, Kosaki K (2007). Embryonic expression profile of chicken CHD7, the ortholog of the causative gene for CHARGE syndrome. Birth defects research. Part A, Clinical and molecular teratology 79(1): 50-57.

Aramaki M, Udaka T, Kosaki R, Makita Y, Okamoto N, Yoshihashi H, Oki H, Nanao K, Moriyama N, Oku S, Hasegawa T, Takahashi T, Fukushima Y, Kawame H, Kosaki K (2006). Phenotypic spectrum of CHARGE syndrome with CHD7 mutations. The Journal of pediatrics 148(3): 410-414.

Bajpai R, Chen DA, Rada-Iglesias A, Zhang J, Xiong Y, Helms J, Chang C-P, Zhao Y, Swigut T, Wysocka J (2010). CHD7 cooperates with PBAF to control multipotent neural crest formation. Nature 463(7283): 958-962.

Batsukh T, Pieper L, Koszucka AM, Velsen N von, Hoyer-Fender S, Elbracht M, Bergman JEH, Hoefsloot LH, Pauli S (2010). CHD8 interacts with CHD7, a protein which is mutated in CHARGE syndrome. Human molecular genetics 19(14): 2858-2866.

Baumann M, Steichen-Gersdorf E, Krabichler B, Muller T, Janecke AR (2017). A recognizable type of syndromic short stature with arthrogryposis caused by bi-allelic SEMA3A loss-of-function variants. Clinical Genetics.

Bergman JE, Janssen N, Hoefsloot LH, Jongmans MC, Hofstra RM, van RavenswaaijArts CM (2011). CHD7 mutations and CHARGE syndrome: the clinical implications of an expanding phenotype. Journal of Medical Genetics 48(5): 334-342. 
Blake KD, Davenport SL, Hall BD, Hefner MA, Pagon RA, Williams MS, Lin AE, Graham JM JR (1998). CHARGE association: an update and review for the primary pediatrician. Clinical pediatrics 37(3): 159-173.

Bosman EA, Penn AC, Ambrose JC, Kettleborough R, Stemple DL, Steel KP (2005). Multiple mutations in mouse Chd7 provide models for CHARGE syndrome. Human molecular genetics 14(22): 3463-3476.

Bradford MM (1976). A rapid and sensitive method for the quantitation of microgram quantities of protein utilizing the principle of protein-dye binding. Analytical biochemistry 72: 248-254.

Bronner ME and LeDouarin NM (2012). Development and evolution of the neural crest: an overview. Developmental biology 366(1): 2-9.

Carmona-Fontaine C, Theveneau E, Tzekou A, Tada M, Woods M, Page KM, Parsons M, Lambris JD, Mayor R (2011). Complement fragment C3a controls mutual cell attraction during collective cell migration. Developmental cell 21(6): 1026-1037.

Chalouhi C, Faulcon P, Le Bihan C, Hertz-Pannier L, Bonfils P, Abadie V (2005). Olfactory Evaluation in Children: Application to the CHARGE Syndrome. Pediatrics 116(1): e81-e88.

Christensen C, Ambartsumian N, Gilestro G, Thomsen B, Comoglio P, Tamagnone L, Guldberg P, Lukanidin E (2005). Proteolytic processing converts the repelling signal Sema3E into an inducer of invasive growth and lung metastasis. Cancer research 65(14): 6167-6177.

Crotti L, Lundquist AL, Insolia R, Pedrazzini M, Ferrandi C, Ferrari GM de, Vicentini A, Yang P, Roden DM, George AL JR, Schwartz PJ (2005). KCNH2-K897T is a genetic modifier of latent congenital long-QT syndrome. Circulation 112(9): 1251-1258.

Delahaye A, Sznajer Y, Lyonnet S, Elmaleh-Berges M, Delpierre I, Audollent S, WienerVacher S, Mansbach AL, Amiel J, Baumann C, Bremond-Gignac D, Attie-Bitach T, Verloes A, Sanlaville D (2007). Familial CHARGE syndrome because of CHD7 mutation: clinical intra- and interfamilial variability. Clinical Genetics 72(2): 112-121. 
Dodé C and Hardelin J-P (2009). Kallmann syndrome. European Journal of Human Genetics 17(2): 139-146.

Dupin E and Sommer L (2012). Neural crest progenitors and stem cells: from early development to adulthood. Developmental biology 366(1): 83-95.

Eickholt BJ, Mackenzie SL, Graham A, Walsh FS, Doherty P (1999). Evidence for collapsin-1 functioning in the control of neural crest migration in both trunk and hindbrain regions. Development (Cambridge, England) 126(10): 2181-2189.

Esselens C, Malapeira J, Colome N, Casal C, Rodriguez-Manzaneque JC, Canals F, Arribas $\mathrm{J}$ (2010). The cleavage of semaphorin 3C induced by ADAMTS1 promotes cell migration. The Journal of biological chemistry 285(4): 2463-2473.

Etchevers HC, Amiel J, Lyonnet S (2006). Molecular bases of human neurocristopathies. Advances in experimental medicine and biology 589: 213-234.

Etchevers HC, Amiel J, Lyonnet S (2007). Genetic and molecular bases of neurocristopathies. Archives de pediatrie organe officiel de la Societe francaise de pediatrie 14(6): 668-672.

Flanagan JF, Blus BJ, Kim D, Clines KL, Rastinejad F, Khorasanizadeh S (2007). Molecular implications of evolutionary differences in CHD double chromodomains. Journal of molecular biology 369(2): 334-342.

Flaus A, Martin DM, Barton GJ, Owen-Hughes T (2006). Identification of multiple distinct Snf2 subfamilies with conserved structural motifs. Nucleic acids research 34(10): 2887-2905.

Gammill LS and Bronner-Fraser M (2003). Neural crest specification: migrating into genomics. Nature reviews. Neuroscience 4(10): 795-805.

Gershoni JM and Palade GE (1983). Protein blotting: principles and applications. Analytical biochemistry 131(1): 1-15.

Gong S-G (2014). Cranial neural crest: migratory cell behavior and regulatory networks. Experimental cell research 325(2): 90-95. 
Gu C, Yoshida Y, Livet J, Reimert DV, Mann F, Merte J, Henderson CE, Jessell TM, Kolodkin AL, Ginty DD (2005). Semaphorin 3E and plexin-D1 control vascular pattern independently of neuropilins. Science (New York, N.Y.) 307(5707): 265-268.

Hall BD (1979). Choanal atresia and associated multiple anomalies. The Journal of Pediatrics 95(3): 395-398.

Hall BK (2000). The neural crest as a fourth germ layer and vertebrates as quadroblastic not triploblastic. Evolution and Development 2(1): 3-5.

Hall JA and Georgel PT (2007). CHD proteins: a diverse family with strong ties. Biochemistry and cell biology = Biochimie et biologie cellulaire 85(4): 463-476.

Hanchate NK, Giacobini P, Lhuillier P, Parkash J, Espy C, Fouveaut C, Leroy C, Baron S, Campagne C, Vanacker C, Collier F, Cruaud C, Meyer V, Garcia-Pinero A, Dewailly D, Cortet-Rudelli C, Gersak K, Metz C, Chabrier G, Pugeat M, Young J, Hardelin J-P, Prevot V, Dode C (2012). SEMA3A, a gene involved in axonal pathfinding, is mutated in patients with Kallmann syndrome. PLoS genetics 8(8): e1002896.

Hittner HM, Hirsch NJ, Kreh GM, Rudolph AJ (1979). Colobomatous microphthalmia, heart disease, hearing loss, and mental retardation. A syndrome. Journal of Pediatric Ophthalmology and Strabismus 16(2): 122-128.

Hofmann K, Zweier M, Sticht H, Zweier C, Wittmann W, Hoyer J, Uebe S, van Haeringen A, Thiel CT, Ekici AB, Reis A, Rauch A (2013). Biallelic SEMA3A defects cause a novel type of syndromic short stature. American journal of medical genetics. Part $A$ 161A(11): 2880-2889.

Ishii M, Arias AC, Liu L, Chen Y-B, Bronner ME, Maxson RE (2012). A stable cranial neural crest cell line from mouse. Stem cells and development 21(17): 3069-3080.

Issekutz KA, Graham JM JR, Prasad C, Smith IM, Blake KD (2005). An epidemiological analysis of CHARGE syndrome: preliminary results from a Canadian study. American journal of medical genetics. Part A 133A(3): 309-317.

Jager R, Maurer J, Jacob A, Schorle H (2004). Cell type-specific conditional regulation of the c-myc proto-oncogene by combining Cre/loxP recombination and tamoxifenmediated activation. Genesis (New York, N.Y. 2000) 38(3): 145-150. 
Janssen BJ, Robinson RA, Perez-Branguli F, Bell CH, Mitchell KJ, Siebold C, Jones EY (2010). Structural basis of semaphorin-plexin signalling. Nature 467(7319): 11181122.

Janssen N, Bergman JEH, Swertz MA, Tranebjaerg L, Lodahl M, Schoots J, Hofstra RMW, van Ravenswaaij-Arts CMA, Hoefsloot LH (2012). Mutation update on the CHD7 gene involved in CHARGE syndrome. Human mutation 33(8): 1149-1160.

Jones NC, Lynn ML, Gaudenz K, Sakai D, Aoto K, Rey J-P, Glynn EF, Ellington L, Du C, Dixon J, Dixon MJ, Trainor PA (2008). Prevention of the neurocristopathy Treacher Collins syndrome through inhibition of p53 function. Nature medicine 14(2): 125-133.

Jongbloets $B C$ and Pasterkamp RJ (2014). Semaphorin signalling during development. Development (Cambridge, England) 141(17): 3292-3297.

Jongmans MC, Admiraal RJ, van der Donk KP, Vissers LE, Baas AF, Kapusta L, van Hagen JM, Donnai D, Ravel TJ de, Veltman JA, van Geurts KA, Vries BB de, Brunner HG, Hoefsloot LH, van Ravenswaaij CM (2006). CHARGE syndrome: the phenotypic spectrum of mutations in the CHD7 gene. Journal of Medical Genetics 43(4): 306-314.

Jongmans MC, Hoefsloot LH, van der Donk KP, Admiraal RJ, Magee A, van de Laar I, Hendriks Y, Verheij JB, Walpole I, Brunner HG, van Ravenswaaij CM (2008). Familial CHARGE syndrome and the CHD7 gene: a recurrent missense mutation, intrafamilial recurrence and variability. American journal of medical genetics. Part $A 146 \mathrm{~A}(1)$ : $43-50$.

Jongmans MC, van Ravenswaaij-Arts CM, Pitteloud N, Ogata T, Sato N, Claahsen-van der Grinten, H L, van der Donk K, Seminara S, Bergman JE, Brunner HG, Crowley WF JR, Hoefsloot LH (2009). CHD7 mutations in patients initially diagnosed with Kallmann syndrome--the clinical overlap with CHARGE syndrome. Clinical Genetics 75(1): 65-71.

Kearney JA (2011). Genetic modifiers of neurological disease. Current opinion in genetics \& development 21(3): 349-353.

Kerosuo $L$ and Bronner-Fraser M (2012). What is bad in cancer is good in the embryo: importance of EMT in neural crest development. Seminars in cell \& developmental biology 23(3): 320-332. 
Keyte A and Hutson MR (2012). The neural crest in cardiac congenital anomalies. Differentiation; research in biological diversity 84(1): 25-40.

Kim H-G, Kurth I, Lan F, Meliciani I, Wenzel W, Eom SH, Kang GB, Rosenberger G, Tekin M, Ozata M, Bick DP, Sherins RJ, Walker SL, Shi Y, Gusella JF, Layman LC (2008). Mutations in CHD7, encoding a chromatin-remodeling protein, cause idiopathic hypogonadotropic hypogonadism and Kallmann syndrome. American journal of human genetics 83(4): 511-519.

Kirby ML and Hutson MR (2014). Factors controlling cardiac neural crest cell migration. Cell adhesion \& migration 4(4): 609-621.

Klostermann A, Lohrum M, Adams RH, Puschel AW (1998). The chemorepulsive activity of the axonal guidance signal semaphorin $D$ requires dimerization. The Journal of biological chemistry 273(13): 7326-7331.

Knecht AK and Bronner-Fraser M (2002). Induction of the neural crest: a multigene process. Nature reviews. Genetics 3(6): 453-461.

Kolodkin AL, Matthes DJ, Goodman CS (1993). The semaphorin genes encode a family of transmembrane and secreted growth cone guidance molecules. Cell 75(7): 1389-1399.

Koppel AM and Raper JA (1998). Collapsin-1 covalently dimerizes, and dimerization is necessary for collapsing activity. The Journal of biological chemistry 273(25): 15708-15713.

Kulesa PM, Bailey CM, Kasemeier-Kulesa JC, McLennan R (2010). Cranial neural crest migration: new rules for an old road. Developmental biology 344(2): 543-554.

Kuriyama $S$ and Mayor $R$ (2008). Molecular analysis of neural crest migration. Philosophical transactions of the Royal Society of London. Series B, Biological sciences 363(1495): 1349-1362.

Laemmli UK (1970). Cleavage of structural proteins during the assembly of the head of bacteriophage T4. Nature 227(5259): 680-685. 
Lalani S, Safiullah AM, Fernbach SD, Harutyunyan KG, Thaller C, Peterson LE, McPherson JD, Gibbs RA, White LD, Hefner M, Davenport SL, Graham JM, Bacino CA, Glass NL, Towbin JA, Craigen WJ, Neish SR, Lin AE, Belmont JW (2006). Spectrum of CHD7 mutations in 110 individuals with CHARGE syndrome and genotype-phenotype correlation. American journal of human genetics 78(2): 303-314.

Layman WS, Hurd EA, Martin DM (2010). Chromodomain proteins in development: lessons from CHARGE syndrome. Clinical Genetics 78(1): 11-20.

Liu H, Juo ZS, Shim AH, Focia PJ, Chen X, Garcia KC, He X (2010). Structural basis of semaphorin-plexin recognition and viral mimicry from Sema7A and A39R complexes with PlexinC1. Cell 142(5): 749-761.

Marfella CG and Imbalzano AN (2007). The Chd Family of Chromatin Remodelers. Mutation research 618(1-2): 30-40.

Martin DM (2015). Epigenetic Developmental Disorders: CHARGE syndrome, a case study. Current genetic medicine reports 3(1): 1-7.

Maurer J, Fuchs S, Jager R, Kurz B, Sommer L, Schorle H (2007). Establishment and controlled differentiation of neural crest stem cell lines using conditional transgenesis. Differentiation; research in biological diversity 75(7): 580-591.

Mayanil CS (2013). Transcriptional and epigenetic regulation of neural crest induction during neurulation. Developmental neuroscience 35(5): 361-372.

Mayor R and Carmona-Fontaine C (2010). Keeping in touch with contact inhibition of locomotion. Trends in cell biology 20(6): 319-328.

Mayor $\mathrm{R}$ and Theveneau E (2013). The neural crest. Development (Cambridge, England) 140(11): 2247-2251.

Messina A and Giacobini $P$ (2013). Semaphorin signaling in the development and function of the gonadotropin hormone-releasing hormone system. Frontiers in endocrinology 4: 133.

Milet C and Monsoro-Burq AH (2012). Neural crest induction at the neural plate border in vertebrates. Developmental biology 366(1): 22-33. 
Mullis K, Faloona F, Scharf S, Saiki R, Horn G, Erlich H (1986). Specific enzymatic amplification of DNA in vitro: the polymerase chain reaction. Cold Spring Harb Symp Quant Biol 51: 263-273.

Neufeld G, Sabag AD, Rabinovicz N, Kessler O (2012). Semaphorins in angiogenesis and tumor progression. Cold Spring Harbor perspectives in medicine 2(1): a006718.

Nogi T, Yasui N, Mihara E, Matsunaga Y, Noda M, Yamashita N, Toyofuku T, Uchiyama S, Goshima Y, Kumanogoh A, Takagi J (2010). Structural basis for semaphorin signalling through the plexin receptor. Nature 467(7319): 1123-1127.

Ogata T, Fujiwara I, Ogawa E, Sato N, Udaka T, Kosaki K (2006). Kallmann syndrome phenotype in a female patient with CHARGE syndrome and CHD7 mutation. Endocrine journal 53(6): 741-743.

Pagon RA, Graham JM JR, Zonana J, Yong SL (1981). Coloboma, congenital heart disease, and choanal atresia with multiple anomalies: CHARGE association. The Journal of pediatrics 99(2): 223-227.

Passos-Bueno MR, Ornelas CC, Fanganiello RD (2009). Syndromes of the first and second pharyngeal arches: A review. American journal of medical genetics. Part $A$ 149A(8): 1853-1859.

Pauli S, Pieper L, Häberle J, Grzmil P, Burfeind P, Steckel M, Lenz U, Michelmann HW (2009). Proven germline mosaicism in a father of two children with CHARGE syndrome. Clinical Genetics 75(5): 473-479.

Pelengaris S, Littlewood T, Khan M, Elia G, Evan G (1999). Reversible activation of cMyc in skin: induction of a complex neoplastic phenotype by a single oncogenic lesion. Molecular cell 3(5): 565-577.

Pinto G, Abadie V, Mesnage R, Blustajn J, Cabrol S, Amiel J, Hertz-Pannier L, Bertrand AM, Lyonnet S, Rappaport R, Netchine I (2005). CHARGE Syndrome Includes Hypogonadotropic Hypogonadism and Abnormal Olfactory Bulb Development. The Journal of Clinical Endocrinology \& Metabolism 90(10): 5621-5626. 
Plein A, Calmont A, Fantin A, Denti L, Anderson NA, Scambler PJ, Ruhrberg C (2015). Neural crest-derived SEMA3C activates endothelial NRP1 for cardiac outflow tract septation. The Journal of clinical investigation 125(7): 2661-2676.

Prasad MS, Sauka-Spengler T, LaBonne C (2012). Induction of the neural crest state: control of stem cell attributes by gene regulatory, post-transcriptional and epigenetic interactions. Developmental biology 366(1): 10-21.

Randall V, McCue K, Roberts C, Kyriakopoulou V, Beddow S, Barrett AN, Vitelli F, Prescott K, Shaw-Smith C, Devriendt K, Bosman E, Steffes G, Steel KP, Simrick S, Basson MA, Illingworth E, Scambler PJ (2009). Great vessel development requires biallelic expression of Chd7 and Tbx1 in pharyngeal ectoderm in mice. The Journal of clinical investigation 119(11): 3301-3310.

Rao MS and Anderson DJ (1997). Immortalization and controlledin vitro differentiation of murine multipotent neural crest stem cells. Journal of neurobiology 32(7): 722-746.

Rondanino C, Bousser M-T, Monsigny M, Roche A-C (2003). Sugar-dependent nuclear import of glycosylated proteins in living cells. Glycobiology 13(7): 509-519.

Rudolph B, Hueber A-O, Evan Gl (2000). Reversible activation of c-Myc in thymocytes enhances positive selection and induces proliferation and apoptosis in vitro. Oncogene 19(15): 1891-1900.

Sanger F, Nicklen S, Coulson AR (1977). DNA sequencing with chain-terminating inhibitors. Proceedings of the National Academy of Sciences of the United States of America 74(12): 5463-5467.

Sanlaville D, Etchevers HC, Gonzales M, Martinovic J, Clement-Ziza M, Delezoide AL, Aubry MC, Pelet A, Chemouny S, Cruaud C, Audollent S, Esculpavit C, Goudefroye G, Ozilou C, Fredouille C, Joye N, Morichon-Delvallez N, Dumez Y, Weissenbach J, Munnich A, Amiel J, Encha-Razavi F, Lyonnet S, Vekemans M, Attie-Bitach T (2006). Phenotypic spectrum of CHARGE syndrome in fetuses with CHD7 truncating mutations correlates with expression during human development. Journal of Medical Genetics 43(3): 211-217.

Sanlaville D and Verloes A (2007). CHARGE syndrome: an update. European journal of human genetics EJHG 15(4): 389-399. 
Schulz Y, Freese L, Manz J, Zoll B, Volter C, Brockmann K, Bogershausen N, Becker J, Wollnik B, Pauli S (2014a). CHARGE and Kabuki syndromes: a phenotypic and molecular link. Human molecular genetics 23(16): 4396-4405.

Schulz Y, Wehner P, Opitz L, Salinas-Riester G, Bongers EM, van Ravenswaaij-Arts CM, Wincent J, Schoumans J, Kohlhase J, Borchers A, Pauli S (2014b). CHD7, the gene mutated in CHARGE syndrome, regulates genes involved in neural crest cell guidance. Human genetics 133(8): 997-1009.

Sekido Y, Bader S, Latif F, Chen JY, Duh FM, Wei MH, Albanesi JP, Lee CC, Lerman MI, Minna JD (1996). Human semaphorins $A(V)$ and IV reside in the 3p21.3 small cell lung cancer deletion region and demonstrate distinct expression patterns. Proceedings of the National Academy of Sciences of the United States of America 93(9): 4120-4125.

Serini G, Valdembri D, Zanivan S, Morterra G, Burkhardt C, Caccavari F, Zammataro L, Primo L, Tamagnone L, Logan M, Tessier-Lavigne M, Taniguchi M, Puschel AW, Bussolino $F$ (2003). Class 3 semaphorins control vascular morphogenesis by inhibiting integrin function. Nature 424(6947): 391-397.

Shellard A and Mayor R (2016). Chemotaxis during neural crest migration. Seminars in cell \& developmental biology 55: 111-118.

Siebert JR, Graham JM JR, MacDonald C (1985). Pathologic features of the CHARGE association: support for involvement of the neural crest. Teratology 31(3): 331-336.

Suzuki K, Kumanogoh A, Kikutani H (2008). Semaphorins and their receptors in immune cell interactions. Nature immunology 9(1): 17-23.

Takahashi T, Fournier A, Nakamura F, Wang LH, Murakami Y, Kalb RG, Fujisawa H, Strittmatter SM (1999). Plexin-neuropilin-1 complexes form functional semaphorin-3A receptors. Cell 99(1): 59-69.

Takamatsu $\mathrm{H}$ and Kumanogoh $\mathrm{A}$ (2012). Diverse roles for semaphorin-plexin signaling in the immune system. Trends in immunology 33(3): 127-135. 
Tamagnone L, Artigiani S, Chen H, He Z, Ming Gl, Song H, Chedotal A, Winberg ML, Goodman CS, Poo M, Tessier-Lavigne M, Comoglio PM (1999). Plexins are a large family of receptors for transmembrane, secreted, and GPI-anchored semaphorins in vertebrates. Cell 99(1): 71-80.

Tamagnone $L$ and Comoglio PM (2004). To move or not to move? Semaphorin signalling in cell migration. EMBO reports 5(4): 356-361.

Theveneau $E$ and Mayor $R$ (2012). Neural crest migration: interplay between chemorepellents, chemoattractants, contact inhibition, epithelial-mesenchymal transition, and collective cell migration. Wiley interdisciplinary reviews. Developmental biology 1(3): 435-445.

Toyofuku T, Yabuki M, Kamei J, Kamei M, Makino N, Kumanogoh A, Hori M (2007). Semaphorin-4A, an activator for T-cell-mediated immunity, suppresses angiogenesis via Plexin-D1. The EMBO journal 26(5): 1373-1384.

Toyofuku T, Yoshida J, Sugimoto T, Yamamoto M, Makino N, Takamatsu H, Takegahara N, Suto F, Hori M, Fujisawa H, Kumanogoh A, Kikutani H (2008). Repulsive and attractive semaphorins cooperate to direct the navigation of cardiac neural crest cells. Developmental biology 321(1): 251-262.

van Meter TD and Weaver DD (1996). Oculo-auriculo-vertebral spectrum and the CHARGE association: clinical evidence for a common pathogenetic mechanism. Clinical dysmorphology 5(3): 187-196.

Verloes A (2005). Updated diagnostic criteria for CHARGE syndrome: a proposal. American journal of medical genetics. Part A 133A(3): 306-308.

Vissers LE, van Ravenswaaij CM, Admiraal R, Hurst JA, Vries BB de, Janssen IM, van der Vliet WA, Huys EH, Jong PJ de, Hamel BC, Schoenmakers EF, Brunner HG, Veltman JA, van Kessel AG (2004). Mutations in a new member of the chromodomain gene family cause CHARGE syndrome. Nature genetics 36(9): 955-957. 
Vuorela P, Ala-Mello S, Saloranta C, Penttinen M, Poyhonen M, Huoponen K, Borozdin W, Bausch B, Botzenhart EM, Wilhelm C, Kaariainen H, Kohlhase J (2007). Molecular analysis of the CHD7 gene in CHARGE syndrome: identification of 22 novel mutations and evidence for a low contribution of large CHD7 deletions. Genetics in medicine official journal of the American College of Medical Genetics 9(10): 690-694.

Williams MS (2005). Speculations on the pathogenesis of CHARGE syndrome. American journal of medical genetics. Part A 133A(3): 318-325.

Winberg ML, Noordermeer JN, Tamagnone L, Comoglio PM, Spriggs MK, TessierLavigne M, Goodman CS (1998). Plexin A is a neuronal semaphorin receptor that controls axon guidance. Cell 95(7): 903-916.

Wincent J, Holmberg E, Stromland K, Soller M, Mirzaei L, Djureinovic T, Robinson K, Anderlid B, Schoumans J (2008). CHD7 mutation spectrum in 28 Swedish patients diagnosed with CHARGE syndrome. Clinical Genetics 74(1): 31-38.

Woodage T, Basrai MA, Baxevanis AD, Hieter P, Collins FS (1997). Characterization of the CHD family of proteins. Proceedings of the National Academy of Sciences 94(21): 11472-11477.

Young J, Metay C, Bouligand J, Tou B, Francou B, Maione L, Tosca L, Sarfati J, Brioude F, Esteva B, Briand-Suleau A, Brisset S, Goossens M, Tachdjian G, Guiochon-Mantel A (2012). SEMA3A deletion in a family with Kallmann syndrome validates the role of semaphorin $3 \mathrm{~A}$ in human puberty and olfactory system development. Human reproduction (Oxford, England) 27(5): 1460-1465.

Zhang D, Ighaniyan S, Stathopoulos L, Rollo B, Landman K, Hutson J, Newgreen D (2014). The neural crest: a versatile organ system. Birth defects research. Part $C$, Embryo today reviews 102(3): 275-298. 


\section{$7 \quad$ Acknowledgements}

I would like to express my gratitude to PD Dr. Silke Pauli for being my referee and supervisor, for given me the opportunity to work on this interesting project and for her guidance and support during the entire process of my PhD study.

I sincerely thank Prof. Steven A. Johnsen, PhD and Prof. Dr. Ahmed Mansouri for being my thesis committee members.

Moreover, I am very thankful to Prof. Dr. Ralf Dressel, Prof. Dr. Thomas Meyer and Prof. Dr. Lutz Walter, who kindly agreed to evaluate my dissertation and participate in the examination.

I thank the Deutsche Forschungsgemeinschaft (DFG) for financing my PhD study.

I would like to thank Dr. Roser Ufartes Mas for her support and helpful suggestions during the last year of my PhD study.

A special thank goes to my lab colleague Johanna Mänz for her patient help and advice and for the cheerful and friendly atmosphere in the lab.

I would like to thank all other current and former colleagues of the Institute of Human Genetics, especially Dr. Nadine Mellies, Dr. Jessica Nolte-Kaitschick and Dr. Yvonne Schulz, for their advice and support as well as the friendly working atmosphere.

I express my heartfelt gratitude to my parents, who always believe in me. You supported me unconditionally with your encouragement and patience throughout my $\mathrm{PhD}$ time. Thanks for your trust and love! Therefore, I dedicate this thesis to you!

Thank you André for always backing me up, putting faith in me, encouraging me and cheering me up, especially during the hard times of my study! 


\section{$8 \quad$ Curriculum vitae}

\section{Personal details}

$\begin{array}{ll}\text { Name } & \text { Luisa Freese } \\ \text { Date of birth } & 03 / 17 / 1986 \\ \text { Place of birth } & \text { Halberstadt, Germany } \\ \text { Nationality } & \text { german } \\ \text { Address } & \text { Haeckelstr. 4, 30173 Hanover }\end{array}$

\section{Education}

Since November 2013

2011-2013

2008-2011
PhD study of Molecular Medicine at the Institute of Human Genetics

Georg-August University Göttingen

Doctoral Thesis: Elucidating the pathomechanism behind the neurocristopathy CHARGE syndrome

Master of Science study of Biomedicine

Hannover Medical School

Master Thesis: Untersuchungen zur Funktion des humanen Testis-spezifischen Proteins, Y-kodiert (TSPY) bei TSPYtransgenen KIT-defizienten Mäusen

Bachelor of Science study of Plant Biotechnology

Gottfried Wilhelm Leibniz University Hannover

Bachelor Thesis: Optimierung der Anwendung von Mikrosatelliten für Diaeretiella rapae und erstes Screening einer Feldpopulation 
2005-2008

Training for Biology Laboratory Technician

Research Center Borstel - Leibniz-Center for Medicine and Biosciences

1996-2005

Secondary School: Käthe-Kollwitz-Gymnasium, Halberstadt

1992-1996

Primary School: Grundschule Anne Frank, Halberstadt 